\title{
SUPERCONDUCTING TRANSITION EDGE BOLOMETER
} AND NOISE IN THIN FILMS

$$
\begin{aligned}
& \text { Nan-Hsiung Yeh } \\
& \text { (Ph.D. thesis) }
\end{aligned}
$$

June 1978

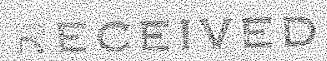

Whatere?

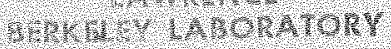

SEP 121978

marnaY ANO HCOWHENS SECTION

Prepared for the U. S. Department of Energy under Contract W-7405-ENG-48

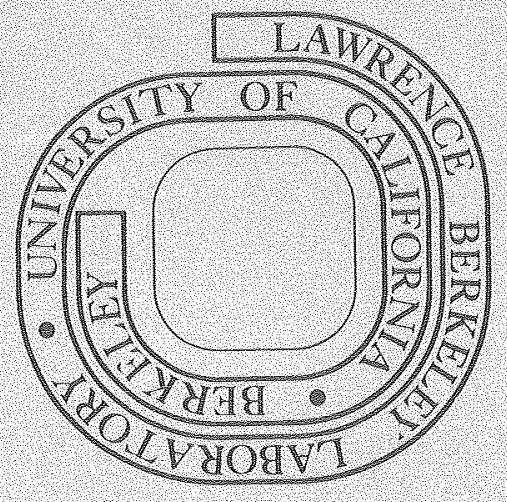

\section{TWO-WEEK LOAN COPY}

This is a Library Circulating Copy which may be borrowed for two weeks. For a personal retention copy, call Tech. Info. Dívision, Ext. 6782 


\section{LEGAL NOTICE}

This report was prepared as an account of work sponsored by the United States Government. Neither the United States nor the Department of Energy, nor any of their employees, nor any of their contractors, subcontractors, or their employees, makes any warranty, express or implied, or assumes any legal liability or responsibility for the accuracy, completeness or usefulness of any information, apparatus, product or process disclosed, or represents that its use would not infringe privately owned rights. 


\title{
SUPERCONDUCTING TRANSITION EDGE BOLOMETER AND NOISE IN THIN FILMS
}

\author{
Nan-Hsiung Yeh
}

\author{
Lawrence Berkeley Laboratory \\ University of California \\ Berkeley, California 94720
}



CONTENTS

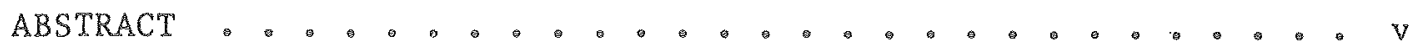

I. INTRODUCTION

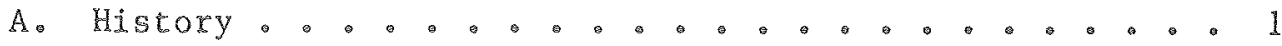

B. Detector Evaluation.................. 2

II. BOLOMETER THEORY . . ................. 9

III. SUPERCONDUCTING TRANSITION EDGE BOLOMETER

A. Principles of Operation ............... 13

B. Experimental Details ............... 13

IV. BOLOMETER NOISES

A. Theoretical Noise Limits , . . . . . . . . 19

B. Experimental Measurements............... 21

V. FAR INFRARED ABSORBER

A. Theory o. . . . . . . . ........... 25

B. Experimental Characterization of Coated Substrates . 28

C. Optical Calibration of Infrared Sources....... 29

D. Far Infrared Efficiency of Bolometer ......... 31

VI. POSSIBLE IMPROVEMENTS .................. 36

VII. THEORY OF THERMAL FLUCTUATION NOISE IN METAL THIN FILMS

A. Introduction ................... 38

B. Equilibrium Temperature Fluctuations . . ...... 39

C. Autocorrelation Function and Decay Measurements . . . 41

VIII. EXPERIMENTAL PROCEDURES

A. Sample Preparation ................ 43

B. SQUID Electronics and Cryostat ........... 45

C. Measurement of Nolse Power Spectrum .......... 49

D. Measurement of Autocorrelation Function from

Macroscopic Decay 。. ................ 51

IX. DISCUSSTON OF THE NOISE EXPERIMENT

A. Thermal Coupling Between Film and Substrate..... 55

B. Normalization of the Decay Curve .......... 60

C. Conclusions..................... 64 
ACKNOWLEDGMENT 。. . . . . . . . . . . . . . . .65

APPENDIX . . . ... .................. 66

REFERENCES ...................... 68

TABLES .......................... 72

FIGURE CAPTIONS ....................... 76

FIGURES ........................... 79 


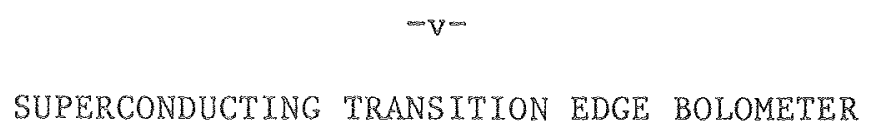

AND NOISE IN THIN FILMS

\begin{abstract}
Nan-Hslung Yeh
Materials and Molecular Research Division Lawrence Berkeley Laboratory and Department of Physics. University of California Berkeley, California 94720
\end{abstract}

\begin{abstract}
We report the development of the composite superconducting transition edge bolometer. The temperature sensitive element is an aluminum strip evaporated onto the sapphire substrate. A bismuth film evaporated on the reverse side of the substrate is used to absorb the submillimeter radiation. The noise limitation of the bolometer is calculated. The fabrication and measured performance are described. The best electrical NEP (noise-equivalentopower) obtained is $(1.7 \pm 0.1) \times 10^{-15} \mathrm{WHz}^{-1 / 2}$ at 2 gz at an operating cemperature of $1.27 \mathrm{~K}$. This NEP is within a factor of 2 of the thermal noise limit. The effective absorptivity of the bismuth film is measured to be $0.47 \pm 0.05$, and the corresponding detectivity $\mathrm{D}^{*}$ is calculated to be $(1.1 \pm 0.1) \times 10^{14} \mathrm{~cm} \mathrm{~W}^{-1} \mathrm{~Hz}^{1 / 2}$. Suggestions are made for further improvements in sensitivity.

The currentodependent noise in thin metal films at the superconducting transition has been further investigated. The measured noise power spectrum of the tin film on sapphire substrate at the superconducting transition is compared with the cosine transforms of the decay curves obtained from scep-function and $\delta$-function thermal perturbations. The nature of the noise driving term is found to be a random current flowing inside the sample, in agreement with the uncorrelated chermal fluctuation model. This result is consistent with the case of a freely
\end{abstract}


suspended tin tiber at the superconducting transition, but in contrast to the room cemperature measuxement where the cosine transform of the step-function response gives the nolse power spectrum, in agreement with the correlated fluctuation model. 


\section{$-1=$ \\ I. INTRODUCTION}

A. HISTORY

Since the discovery of infrared radiation in the eighteenth century, a new field of physlcs has been developed. Various detecting methods were used to sense the invisible infrared radiation. The earliest version of the infrared detector was a mercury thermometer in which the mercury expanded when it absorbed infrared radiation. This method was, of course, a very crude one. Later themopiles, radiometers, bolometers, and photon detectors were developed. These greatly improved man's knowledge of infrared radiation. In World War II infrared detectors were used for night searching, night driving, and detecting hot objects. The military applications caused great advances in infrared technology.

The infrared radiation has expanded the range of wavelengths in spectroscopy. The change of quantum states in rotational and vibrational modes in molecules falls in the infrared range. Infrared spectroscopy has played an important part in determining the structure of molecules. The molecules may also be identified by this emission or absorption spectrum. In pollution experiments, infrared spectroscopy has become a standard technique. Infrared astronomy is one of the most important applications in infrared history. In the last decade many discoveries have been made in this fleld. These experiments made use of groundbased, balloon-borne, and aircraft telescopes. Due to the weakness of the signal, cooled infrared detectors were used to obtaln a better signal-townoise ratio. Recently scientists have become more and more 
interested in cooled infrared telescopes. The use of an infrared detector with high sensitivity was essential to obtaln the full benefit from a cooled telescope. An increase in the sensitivity of the measuring facilities by a factor of 10 would reduce the integration time by a factor of 100 . In the Ear-infrared region (40- $1000 \mu \mathrm{m})$ a good detector is especially valuable to sense the very weak signals.

\section{B. DETECTOR EVALUATTON}

There are two main groups of infraced detectors. The first type is the thermal detector. This employs materials possessing some strongly temperature-dependent property. The incident radiation raises the temperature of the detecting element producing a change in the property used to detect the infrared radiation. Bolometers and thermopiles belong to this group. The second group is the photon detector. The infrared radiation induces an electronic rransition in the detecting element and leads to a change in conductivity or to an output voltage appearing across the terminals of the device. The photon detector responds only to photons of energy greater than the minimum energy required to excite the electronic transition. Photoconductors and photovoltalc devices are examples in the second group.

For ideal infrared detectors, that is, one in which the detecting element and electronics do not incroduce any excess noise to the output, fluctuations in blackbody radiation will limit the detector performance. This sets a minimum detectable signal. No real detector ever satisfies this condition, but the best modern detectors can approach this ideal Iimit to whin a factor of two or three under certain circumstances. 
In a stream of radiation emitted from a blackbody at temperature $\mathrm{T}$, the statistical fluctuation of the number of photons in a given quantum state with frequency $v$ is 1 .

$$
\overline{\Delta \pi^{2}}=\frac{n(\nu) e^{h \nu / k T}}{e^{h \nu / k T}-1} d \nu
$$

When the radiation is incident on the thermal detector, the corresponding NEP (the amount of power which gives the ratio of signal to rms noise per square root unit bandwidth equal to 1) is $\mathrm{s}^{2}$

$$
(N E P)_{T h}^{2}=\frac{4(k T)^{5} A}{c^{2} h^{3}}\left(\int \cos \theta d \Omega\right) \delta_{0}^{\infty} \frac{x^{4} e^{x}}{\left(e^{x}-1\right)^{2}} f(x) d x,
$$

where $f \cos \theta d \Omega$ is the field of view: $x=h c / k T \lambda$; A is detector area; $f(x)$ is the deviation of the spectrum of absorbed power from the blackbody radiation. For blackbody radiation and a black detector, $f(x)=1$. If the detector is in thermal equilibrium with the radiation field, there will be an equal fluctuation in the power radiated by the detector. The above (NEP) th will then be increased by $\sqrt{2}$.

The corresponding photon fluctuation noise for a photon detector operated near its threshold wavelength is

$$
(N E P)_{p h}^{2}=\frac{4 \pi A \varepsilon(k T)^{3}}{\lambda_{c}{ }^{2} h}(f \cos \theta d \Omega) \delta_{x}^{\infty} \frac{x^{2} e^{x}}{\left(e^{x}-1\right)^{2}} d x
$$

where $\varepsilon$ is the quantum efficlency: $\lambda_{c}$ is the cut-off wavelength;

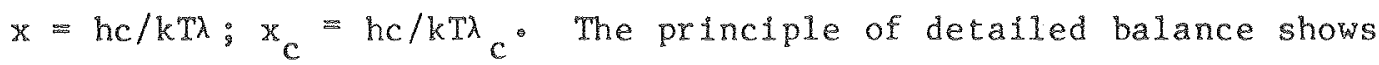
that even if the photoconductor is at a different temperature from the background, the recombination of the photon-excited electrons will be responsible for a fluctuation equal to that given by Eq. (1.3)。 The total (NEP) ph will be increased by $\sqrt{2}$. For photovoltaic or 
photomagnetic devices, Eq. (1.3) still applies.

Both (NEP) ${ }_{\mathrm{th}}$ and $(\mathrm{NEP})_{\mathrm{ph}}$ decrease as the temperature is lowered. The detector performance is usually better at low temperatures. From Eq. (1.2) and $E q \cdot(1.3)$, we can see that (NEP) th is always greater than (NEP) ph (assuming $f(x)=c=1$ ). The background nolse linited detectivity $\left(D^{*}=\varepsilon \sqrt{A} /(N E P)\right)$ for the photon detector is higher than for the thermal detector. Thus photon detector seems to be the better one in the ideal case. However, the real infrared detectors have additional noises from the detecting element and the associated electronics. The actual performance is usually limited by other fluctuations.

For themal detectors, the sensitivity is further limited by the equilibrium temperature fluctuation of the detecting element. l/f noise is another noise source that becomes important at low chopping frequencies. But it can be quenched under certain circumstances. ${ }^{3}$ By increasing the responsivity, the Johnson noise level can be made smaller than other noises. The preamplifier noise can be minimized by choosing a low noise preamplifier and matching the impedance through a txansformex or a tank cixcult. The spectral response of the thermal detector is flat over a wide frequency range. The actual roll-off is determined by the absorption characteristics of the detector material or the transmission of the window. The superconducting bolometer ${ }^{4}$ and the semiconducting bolometer are the most developed ones. The composite aluminum superconducting transtition edge bolometer, 5 which is composed of a low heat capacity sapphre substrate with aluminum film biased at the superconducting transition as the temperature sensor and 
bismuth $11 \mathrm{~m}$ as infrared absorber, may achleve an NEP $=1.7 \times 10^{-15}$ $\mathrm{WHz}^{-1 / 2}$ at $1.27 \mathrm{~K}$. The composite $\mathrm{SNS}^{6,7}$ and the composite $\mathrm{Ge}: \mathrm{Ga}^{8}$ bolometers have the same configurations as the composite aluminum bolometer except the temperature sensors. The SNS bolometer has a superconductor - normal metal - superconductor junction as the themometer. It makes use of the temperature-dependent critical current of the junction to measure the temperature. Due to the low impedance of the junction, a SQUTD has to be used to read out the signal. The best NEP obtained is $5 \times 10^{-15} \mathrm{WHz}^{-1 / 2}$. The Ge:Ga bolometer uses $\mathrm{Ge}: \mathrm{Ga}$ single crystal as a temperature sensor. It is easy to match the impedance to a room temperature preamplifier. However, it suffers $1 / f$ noise at low chopping frequencies. The best NEP meausred is approximately $5 \times 10^{-15} \mathrm{WHz}^{-1 / 2}$, which is comparable with the SNS boloneter.

The photon detector has excess noises due to the thermal excitation of electrons, filling of shallow traps, warming of the detector, reestablishment of a bias condition when the background loading changes, etc. Some of the detectors have multiple time constants, which make the analysis of responsivity much more complicated. In addition, the 1/f notse appearing in the photon detectors is still not well understood yet. So far there is no efficient way to suppress this noise. The spectral response of the photon detector is not flat. Beyond the cut-off wavelength $\lambda_{c}$, no photon absorption occurs. Below $\lambda_{c}$, the performance starts to degrade as the wavelength is decreased. The optimum point is near $\lambda_{\mathrm{c}}$. Furthermore, the intrinsic photon detectors have a cut - of $f$ wavelengh $<20 \mathrm{~mm}^{9}$ The development of extrinsic detectors has increased the cut-off wavelength up to $350 \mu \mathrm{m}$. The Ge:Be 
photoconductor has a cut-off wavelength $\approx 60 \mu \mathrm{m} .10$ The measured NEP is $2 \times 10^{-16} \mathrm{~W} / \sqrt{\mathrm{Hz}}$ with background loading $\approx 9 \times 10^{8}$ photons $/ \mathrm{sec} / \mathrm{cm}^{2}$. The Ge:Ga photoconductor has $\lambda_{c} \approx 120 \mu \mathrm{m}, 10$ NEP $\approx 4 \times 10^{-17} \mathrm{~W} / \sqrt{\mathrm{Hz}}$ for a background loading $3.7 \times 10^{8}$ photons $/ \mathrm{sec} / \mathrm{cm}^{2}$. The n-type GaAs photoconductor ${ }^{11}$ has fairly high cut-off wavelength $(\approx 350 \mu \mathrm{m})$. But so far the measured NEP is not very $\operatorname{good}\left(\approx 2 \times 10^{-13} \mathrm{~W} / \sqrt{\mathrm{Hz}}\right)$. Recently the development of unlaxially stressed Ge:Ga photoconductor ${ }^{12}$ has raised the cut-off wavelength to about $200 \mu \mathrm{m}$. The preliminary measurement showed an NEP about $2 \times 10^{-11} \mathrm{~W} / \sqrt{\mathrm{Hz}}$ at $190 \mu \mathrm{m}$. Further improvement is still in progress. The most recent result ${ }^{13}$ showed that the NEP has achieved $10^{-16} \mathrm{~W} / \sqrt{\mathrm{Hz}}$.

Another far infrared detector which is worth mentioning is the Insb detector. ${ }^{2}$ The far infrared radiation excites the conduction electrons in InSb and changes the electron mobility. Due to the long electron-phonon relaxation time, the electrons may stay "hot" without heating up the lattice. Thus the response time is short ( $<1 \mu s)$. The responsivity falls as $\lambda$ is reduced below $1 \mathrm{~mm}$ so that for applications at wavelength below $500 \mu \mathrm{m}$ the performance is significantly degraded. By applying a magnetic field or using heavily compensated material, the spectral response can be improved at the expense of increasing the NEP. The best NEP obtained for InSb decector is $1.3 \times 10^{-13} \mathrm{~W} / \sqrt{\mathrm{Hz}} \cdot 14$

There are many other room temperature far-infrared detectors. The more important ones are Golay cell, pyroelectric detector, Metal-oxide metal point contact detector, ferroelectric detector, and pyromagnetic detector. The Nernst effect in mixed crystals of InSb-NiSb, ${ }^{15}$ 
$\mathrm{Cd}_{3} \mathrm{As}_{2}$-InAs, ${ }^{16}$ and bismuth and bismuth-antimony alloys ${ }^{17}$ has also been considered for infrared detectors. But, due to the background radiation noise, these detectors can never compete with the ${ }^{4}$ He temperature ones in sensitivity. They certainly are convenient in many applications which do not need very high sensitivities.

We summarize the performance of some of the state-of-the-art far-infrared detectors operating at liquid 4 he temperatures. In Figure 1, specific detectivity $D^{*}(\equiv \sqrt{\mathrm{A}} /(\mathrm{NEP})$ optical $)$ is plotted vs. wavelength. Table 1 shows the corresponding measurement conditions. The performance of InSb detector is not directly related to the detector area. The NEP of stressed Ge:Ga photoconductor was measured in an integrating cavity to enhance the optical efficiency. We have plotted $D\left(=1 /(N E P)_{\text {optical }}\right)$ instead of $D^{*}$ for these two detectors in Fig. 1 . We can see that Al superconducting transition edge bolometer has the highest $D^{*}\left(=1.1 \times 10^{14} \mathrm{cmW}^{-1} \mathrm{~Hz}^{1 / 2}\right)$ in the $200 \sim 1000 \mu \mathrm{m}$ range. In the following chapters we are going to discuss this bolometer in more detail. Chapter II is a description of the general bolometer theory. Chapter III is concerned with the design, fabrication, and operation of Al superconducting transition edge bolometer. Chapeer IV is a discussion of the detector noises. The measured noise power spectrum is compared with the theoretical estimates. The measured electrical NEP $1.7 \times 10^{-15} \mathrm{WHz}^{-1 / 2}$ at $2 \mathrm{~Hz}$ is within a factor of 2 of the thermal noise limit. Chapter $V$ describes measurements of the submillimeter wave absorptivity of Bi-coated sapphire substrate and of the optical efficiency of completed bolometers. The theoretical prediction of $50 \%$ optical efficiency for a bolometer designed for broad-band 
applications has been verifled experimentally for frequencies in the vicinity of $20 \mathrm{~cm}^{-1}$. 


\section{BOLOMETER THEORY}

Consider a bolometer with heat capacity $C$ at temperature $T$ connected to a heat sink at temperature $T_{S}$ via a thermal conductance $G$. The thermal time constant, $\tau$, is $C / G$. In the case of a composite bolometer, the substrate absorbs a fraction $\varepsilon(\leq 1)$ of the incident signal power, $P_{S}$, and the resulting increase in temperature is detected by a separate temperature-sensitive element (thermometer) attached to the substrate. This element is biased with a constant current I that generates a voltage $V$ across it. If the signal is chopped at a frequency $\omega / 2 \pi$, the responsivity $S(\omega)$ is given by ${ }^{1}$

$$
S \equiv \frac{\partial V}{\partial P_{S}}=\frac{\varepsilon \partial V / \partial T}{G-I \partial V / \partial T+i \omega C}=\frac{\varepsilon \partial V / \partial T}{G_{e}(I+i \omega T)}
$$

In $E_{q} \cdot(2.1), G_{e}=(G-I \partial V / \partial T)$ is the effective thermal conductivity, and $\tau_{e}=C / G_{e}$ is the effective thermal time constant. To avoid themal runaway of the bolometer, we require $G_{e}>0$. If $\partial V / g T<0$ (negative thermal feedback), $G_{e}$ is always positive, whereas if av/aT > 0 (positve thermal feedback), the bias current must satisfy the condition $I<G /(\partial V / \partial T)$

The square of the electrical NEP (per unit bandwidth) can be written as the sum of squares of statistically independent terms which arise from a number of sources: 18

$$
\begin{gathered}
(N E P)^{2}=8 k_{B} T_{B} E P_{B}+4 k_{B} T^{2} G+J_{1 / f} /|S|^{2}+4 k_{B} T R /|S|^{2}+ \\
J_{A}(E) /|S|^{2}+(N E P)_{M}^{2}
\end{gathered}
$$

The first term arises from fluctuations in the background blackbody 
power $\& \mathbb{P}_{B}$ absorbed by the bolometer. We assume that the background temperature $\mathrm{T}_{\mathrm{B}} \gg \mathrm{T}$, and that cooled filters are used which limit the background power to wavelengths $\lambda>h c / k_{B} T_{B}$ where the Rayleigh-Jeans limit is valid. The second term axises from the random exchange of energy between the bolometer and the heat sink via the thermal conductance. No treatment has been given of the fluctuations of a bolometer using correct nonequilibrium thermodynamics in the presence of thermal feedback. Consequenty we have used in Eq. (2.2) the conventional approximate expressions dexived from equilibrium thermodynamics. The third term arises from voltage noise in the thermometer which has an 1/f spectral density $J_{1 / f}(f)$. The fourth term is the contribution of Johnson noise in the thermometer which has a resistance $R$. This term is slightly modified for a Josephson junction. The fifth term is the contribution of the amplifier that detects voltage changes across the thermometer. Here $J_{\mathrm{A}}(E)$ is the spectral density of the amplifier voltage noise. The last term is from miscellaneous sources, such as temperature fluctuations in the helium bath, microphony of the bolometer, and pick-up from radio and television stations. One attempts to reduce the noise from these sources to a level below that of the first or second term. If the resistance of the bolometer is measured with an ac bias current and the ac voltage is subsequently lock-in amplified, additional numerical factors are introduced into Eq. (2.2). The first three terms arise from resistance fluctuations in the themometer, and are treated by the detection system in exactly the same way as the signal. On the other hand, the fourth and fifth noise sources do not involve 
resistance fluctuations, and are treated differently by the lock-in amplifier. A proper consideration ${ }^{19}$ of the lock-in amplification shows that the (NEP) $)^{2}$ due to Johnson noise and amplifier noise should be increased by a factor of 2. Depending on their origin, the miscellaneous noise contributions to the (NEP) ${ }^{2}$ may or may not contain this factor. Eq. (2.2) also reveals that the background and thermal fluctuation (NEP) ${ }^{2}$ (first and second terms) are independent of the value of wt $e^{\text {s However, }}$ the third, fourth, and fifth terms are proportional to $\left(1+\omega^{2} \tau_{e}^{2}\right)$, and therefore increase as $\omega^{2}$ for frequencies higher than $1 / 2 \pi \tau \mathrm{e}^{\circ}$ We may draw several general conclusions about the optimization of bolometers from the form of Eqs. (2.1) and (2.2). First, the temperature $T_{S}$ of the heat sink should be as low as possible. The lower limit is usually fixed by the type of cryostat used. The rapid dependence of important bolometer parameters such as $R$ and $C$ on temperature suggests that the operating temperature of an optimized bolometer will be confined to a small range above $\mathrm{T}_{\mathrm{S}}$. The operating temperature is further restricted in the case of the transition-edge bolometer by the transition temperature of available superconductors. The temperature rise of the bolometer is $T-T_{S}=\left(\varepsilon P_{B}+I V\right) / G$. For bolometer applications in which the absorbed background power $\varepsilon P_{B}$ is large, the value of $G$ required to keep the bolometer cold can be sufficiently large that $G \gg \omega C$. In this limit, $C$ does not appear in $\mathrm{Eq} \cdot(2.1)$ or $(2.2)$. In the low background limit, on the other hand, $C$ should be made as small as possible. The value of $\tau_{e}$ is chosen after considering the requirements

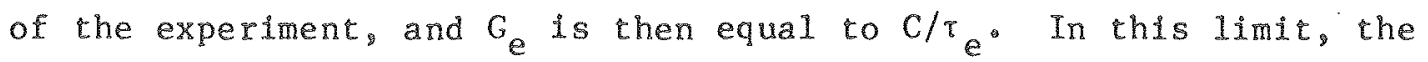
bolometer NEP varies as $\mathrm{C}^{1 / 2}$. This dependence is Immediately clear 
if the NEP is IImited by thermal fluctuation noise (the second term in Eq. (2.2)). It is also true if the NEP is limited by any of the subsequent terms in $\mathrm{Eq} \cdot(2.2)$ which vary as $|S|^{-2} \approx G^{2} / I(\partial R / \partial T)$. Since the largest useful values of I are limited by bolometer heating to $I \approx G\left(T-T_{S}\right) / V$, we see that the optimum $|S|^{-2} \propto G$. Although the bolometers described in this paper are of value in both high background and low background limits, bolometers were constructed only with parameters optimized for low background applications. 


\section{SUPERCONDUCTING TRANSITION EDGE BOLOMETER}

\section{A. PRINCIPLES OF OPERATION}

The transition-edge bolometer consists of a thin film of superconductor (aluminum in the present work) evaporated onto a suitable substrate. The temperature of the bolometer is maintained close to the mid-point of the superconducting transition where the resistance $R$ of the film increases rapidly with increasing temperature. An ac current of rms amplitude $I$ is passed through the film so that changes in temperature generate changes in the ac voltage that are detected by a low noise amplifier. The electrical responsivity is then given from Eq. (2.1) as $S=I(\partial R / \partial T) / G_{e}\left(1+i \omega e_{e}\right)$. The responsivity increases with increasing bias current but, since the thermal feedback is positive, $I^{2}$ must be less than $G /(\partial R / \partial T)$. If we take as typical

values $G=5 \times 10^{-8} \mathrm{WK}^{-1}$ and $\partial \mathrm{R} / \partial \mathrm{T}=10^{3} \mathrm{\Omega K}^{-1}$, this requirement implies that $I<7 \mu \mathrm{A}$. For example, if we choose $I=1 \mu \mathrm{A}, S \approx 2 \times 10^{4} \mathrm{VW}^{-1}$ at zero frequency.

\section{B. EXPERIMENTAL DETAILS}

The bolometers were fabricated on single-crystal sapphire substrates with dimensions $4 \times 4 \times 0.135 \mathrm{~mm}, 4 \times 4 \times 0.050 \mathrm{~mm}$, or $4 \times 2 \times 0.135 \mathrm{~mm}$ (see Fig. 1). Four rectangles of In of size $0.7 \times 0.15 \times 0.0005 \mathrm{~mm}$ were evaporated onto the corners of the substrate, followed by $5 \mathrm{~nm}$ of $\mathrm{Cu}$. (The thin $\mathrm{Cu}$ layer appears to stabilize the In-Al interface and to inhibit the growth of an oxide layer.) An Al strip $0.25 \mathrm{~mm}$ wide and $50 \mathrm{~nm}$ to $150 \mathrm{~nm}$ thick (the superconducting thermometer) was evaporated at a pressure of $\sim 2 \times 10^{-7}$ Torr and a rate of $\sim 20 \mathrm{~nm} \mathrm{~s}^{-1}$ 
near one edge of the substrate, and a BI strip 0.5 mm wide and $0.1 \mu \mathrm{m}$ thick (the heacer) was evaporated near the opposing edge Each end of the strips made good electrical contact with an In/Cu rectangle. The edges of the Al strip were cut with a diamond knife to reduce the width of the superconducting transition. ${ }^{20}$ The transition widh was typically $3 \mathrm{mK}$. The transition temperature of the films varied from $\sim 1.2 \mathrm{~K}$ for the thicker samples to $\sim 1.4 \mathrm{~K}$ for the thinner samples. At low temperatures, the resistance of a $50 \mathrm{~nm}$ thick Al film in the normal state was 2 to 4 \&, while the resistance of the Bi film was about $1500 \Omega$ s

Four small pieces of lead foll were attached with epoxy to an OFHC copper mount as show in Fig. 2. (OFHC coppex was used to minimize the thermal equilibration time of the mount.) The epoxy provided elec trical insulation between the foils and the mount. Nylon threads with a diameter of $~ 15 \mu m$ were separated from a multifilament thread, and cleaned with trichloroethylene Two threads were attached to the lead foils with GE7031 varnish as shown. An indium film about 2 um thick was evaporated on the In/Cu rectangles on the substrate and on the corresponding positions on the nylon threads. The substrate was then carefully pressed onto the threads so that the indium films were cold-welded together. We found that this assembly technique was highly reliable, and that the bolometer could be thermally cycled repeatedly with no tendency for the nylon threads to become detached. The remaining portions of the threads were coated with 0.75 um of indium to provide thermal and superconducting electrical connections to the thermometer and heater. This thickness gave a thernal conductance of about 
$5 \times 10^{-8} \mathrm{WK}^{-1}$ at the operating temperature of the bolometer. A $0.08 \mathrm{~mm}$ bismuth film was evaporated on the back of the substrate as a far infrared absorber.

The mount was suspended from the top-plate of a vacuum can by four 1-72 nylon screws about $10 \mathrm{~mm}$ long. Below the mount was attached a block of copper (volume $55 \times 10^{3} \mathrm{~mm}^{3}$ ) on which was wound a $1 \mathrm{k} \Omega$ manganin heater. This arrangement provided a low-pass thermal filter to reduce the effects of temperature fluctuations in the helium bath. The thermal time-constant of the block and bolometer mount was about 20 s. A vacuum-tight sapphire window was sealed into the top-plate of the vacuum can above the bolonter. A stainless steel light-pipe with an i.d. of about $12 \mathrm{~mm}$ connected the sapphire window to the top of the cryostat. The light-pipe was closed at the top with a black polyethylene sheet $200 \mu \mathrm{m}$ thick. The vacuum can was evacuated through a $3 \mathrm{~mm}$ stainless steel tube that contained a radiation baffle. Leads from the bolometer circuit were brought out through a vacuum seal in the topoplate.

The Al strip formed one arm of a Wheatstone bridge, each of the other three arms being a manganin wire resistor (see Fig. 3). The bridge was operated at $1 \mathrm{kHz}$. The output voltage from the bridge was amplifled by a Triad $G-4$ transformer with a turns ratio of $1: 350$. The $\mu$-metal can had been removed from the transformer which was mounted, together with the manganin resistors, outside the vacuum can. The output of the transformer was connected to a room-temperature FET preamplifiex. The cooled transformer-preamplifier combination achieves a noise temperature of about $1 \mathrm{~K}^{21}$ The preamplifier had a gain of 
$10^{4}$ and was followed by a tuned amplifier, and a lock-1n decector referenced to the $1 \mathrm{kHz}$ oscillator. The output from the lock-in was available via a 1 kHz notch-filter, and was also connected to a feedback circuit that regulated the temperature of the boloneter. It is, of course, essential that the Al strip be maintained on the superconducting transition. Both the temperature of the helium bath and the level of the background radiation contain drifts and low Erequency fluctuations (whose spectral densities vary approximately as $1 / f^{2}$ at low frequencies) sufficiently large to drive the temperature of the bolometer well away from the transition temperature in the absence of temperature regulation. The temperature of the bolometer was regulated by feedback through an amplifier that fed current into the manganin heater on the copper block. In operation, the temperature of the helium bath was about $1.1 \mathrm{~K}$, at least $0.1 \mathrm{~K}$ below the transition temperature of the Al. The feedback circuit supplied the appropriate current to the heater to raise the temperature of the bolometer until the resistance of the Al strip was $1 \Omega$. The response of the feedback circuit was small at the modulation frequency $(\gtrsim 1 \mathrm{~Hz})$ so the amplified signal at the modulation Erequency was avalable at the output. A slow drift in the tempexature of the helium bath or in the amount of background power, on the other hand, was compensated so as to keep the bolometer at its operating temperature.

rypically, $\tau$ is $10^{-2}$ to $10^{-1} \mathrm{~s}$, and the same time constant of the copper mount, $\tau_{m}$, is $\sim 20 \mathrm{~s}$. Thus $\tau \ll \tau_{\mathrm{m}}$. Let $\alpha$ be the loop gain of the feedback system, typically 50. Consider fluctuations 
$\Delta \mathrm{P}_{\mathrm{B}} \cos \left(\omega t+\delta_{1}\right)$ in the background radiation and $\Delta \mathrm{T}_{\mathrm{He}} \cos \left(\omega t+\delta_{2}\right)$ in the temperature of the helium bath at frequency $\omega_{\text {. Here }} \delta_{1}$ and $\delta_{2}$ are arbitrary phase factors. Astraightforward analysis using standard feedback theory shows that the change in the temperature of the bolometer is ${ }^{+}$

$$
\Delta T=\frac{\left(\varepsilon \Delta \mathrm{P}_{B} / G\right)\left(1+\omega^{2} T_{m}^{2}\right)^{1 / 2} \cos \left(\omega t+\delta_{1}\right)+\Delta T_{H e} \cos \left(\omega \tau+\delta_{2}\right)}{\tau \tau_{m}\left[\left(\omega^{2}-\omega_{0}^{2}\right)^{2}+\omega^{2} / \tau^{2}\right]^{1 / 2}}
$$

In $\mathrm{Eq} \cdot(3.1) \omega_{0} \sim\left(\alpha / \tau \tau_{\mathrm{m}}\right)^{1 / 2} \sim 10 \mathrm{~Hz}$ for typical values. Clearly, $\tau^{-1}>\omega_{0}>\tau_{m}^{-1}$. For a frequency $\omega \ll \tau_{m}^{-1}, E q \cdot(3,1)$ simplifies to

$$
\begin{gathered}
\Delta T=\left[\varepsilon \Delta P_{B} / G \alpha\right] \cos \left(\omega t+\delta_{1}\right)+\left[\Delta \mathrm{T}_{\mathrm{He}} / \alpha\right] \cos \left(\omega t+\delta_{2}\right) \\
\left(\omega \ll \tau_{\mathrm{m}}^{-1}\right) .
\end{gathered}
$$

We see that the feedback reduces the effect of either rype of fluctuation by a factor $\alpha \approx 50$. For a frequency $\omega \gg \tau^{-1}$,

$$
\begin{gathered}
\Delta T \equiv\left(\varepsilon \Delta P_{B} / G \omega \tau\right) \cos \left(\omega t+\delta_{1}\right)+\left(\Delta T_{H e} / \omega^{2} \tau \tau_{m}\right) \cos \left(\omega t+\delta_{2}\right) \\
\left(\omega \gg \tau^{-1}\right) .
\end{gathered}
$$

In this limit, the change in the temperature of the bolometer is determined by the appropriate low pass themal filters, and the feedback circuit plays no role.

In Fig. 4 we plot a calculation of the relative squared response $(\Delta T)^{2}$ vs. frequency for fluctuations: (a) $\left(\Delta \mathrm{P}_{B}\right)^{2} \cos ^{2}\left(\omega t+\delta_{1}\right)$ and (b) $\left(\Delta \mathrm{T}_{\mathrm{He}}\right)^{2} \cos ^{2}\left(\omega t+\delta_{2}\right)$. The continuous line is the response without

+ See Appendix. 
feedback, and the dashed 11ne is the response in the presence of feedback. The following parameters were used: $\bar{E}=1, \mathrm{C}=10^{-9} \mathrm{JK}^{-1}$, $G=5 \times 10^{-8} \mathrm{WK}^{-1}, T_{\mathrm{m}}=20 \mathrm{~s}$, and $\alpha=50$. At low Erequencies, the effect of the perturbation on $(\Delta T)^{2}$ has been reduced by a factor of $\alpha^{2} \approx 2,500$ by the feedback, whereas at high frequencies, the feedback has no effecto 


\section{BOLOMETER NOISES}

\section{A. THEORETICAL NOISE LIMITS}

A signal power $\mathrm{P}_{\mathrm{S}} \cos$ wt absorbed by, or generated on, the bolometer produces a voltage $V$ coswt across the bridge, where

$$
V=\sqrt{2} I(\partial R / \partial T) P_{S} / G_{e}\left(1+\omega^{2} \tau_{e}^{2}\right)^{1 / 2} .
$$

The correction $(\sim 10 \%)$ due to the attenuation of the signal by the bridge circuit has been neglected.

We now estimate the contributions of the various noise sources to the electrical NEP for typical values of the parameters, in order to determine which of the sources axe most important. We consider only the case wt $\ll 1$, and assume throughout that $\mathrm{T}=1.2 \mathrm{~K}, \mathrm{G}=5 \times 10^{-8} \mathrm{WK}^{-1}$, $R=1 \Omega$, and $(\partial R / \partial T)=10^{3} \mathrm{~K}^{-1}$. The thermal noise contribution is $(\mathrm{NEP})_{\mathrm{Th}}=\left(4 \mathrm{k}_{\mathrm{B}} \mathrm{T}^{2} \mathrm{G}\right)^{1 / 2} \approx 2 \times 10^{-15} \mathrm{WHz}^{-1 / 2}$. The $1 / \mathrm{f}$ notse in tin films on glass substrates of the superconducting transition is predicted to have a special density ${ }^{3,22}$

$$
S_{1 / F}(E)=\frac{J^{2}(\partial R / \partial T)^{2} k_{B} T^{2}}{C_{F}\left[3+2 \ln \left(\ell_{1} / l_{2}\right)\right] E}
$$

where $\ell_{1}, l_{2}$, and $C_{F}$ are the length, width, and heat capacity of the film, and $J$ is the constant current bias. For the bolometer in a bridge circuit, Eqs. $(4.1)$ and $(4.2)$ lead to

$$
(\operatorname{NEP})_{1 / \mathrm{f}}=G_{e}\left\{\mathrm{k}_{\mathrm{B}} \mathrm{T}^{2} / C_{\mathrm{F}}\left[3+2 \ln \left(\ell_{1} / \ell_{2}\right)\right] \mathrm{f}\right\} 1 / 2 \text {. }
$$

(NEP) $1 / f$ is independent of $I$. If we take the film dimensions as $4 \times 0.25 \times 10^{-4} \mathrm{~mm}$ and use $C_{F}(\mathrm{Al})=1.6 \times 10^{-4} \mathrm{JK}^{-1} \mathrm{~cm}^{-3}$ at $1.2 \mathrm{~K}$ 
we find that at $10 \mathrm{~Hz}(\mathrm{NEP})_{1 / \mathrm{f}} \approx 10^{-14} \mathrm{WHz}^{-1}$. This estimate is a

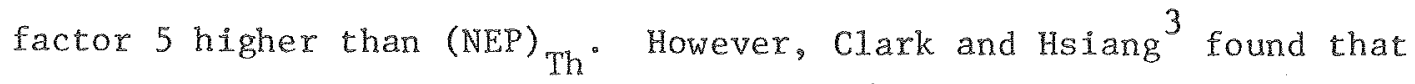
a $5 \mathrm{~nm}$ film of Al deposited on the glass or sapphire substrate prior to the evaporation of the $\mathrm{Sn}$ caused the power spectrum to flatten at Low frequencies. At $10 \mathrm{~Hz}$ the power spectrum was reduced by a factor of 20 or more. Thus if a comparable flattening of the noise power spectrum occurs with Al film on sapphire, $\left(\right.$ NEP) ${ }_{1 / f}$ is expected to be comparable with (NEP) ${ }_{\text {Th }}$ It is difficult to make a priori estimates of the low frequency noise in this regime, and one should not assume from the outset that this contribution is negligible.

The Johnson noise contribution is found by equating the signal voltage $V$ from Eq. (4.1) with $\left(16 k_{B} T R\right)^{1 / 2}$ (the extra factor of 2 arises from the demodulation scheme):

$$
(N E P)_{J}=G_{e}\left(8 k_{B} T R\right)^{1 / 2} / I(\partial R / \partial T)
$$

(NEP) $J$ decreases as $I$ is increased. We can operate the bolometer at a current amplitude (say $2 \mu \mathrm{A}$ ) at which the Johnson noise is negligible compared with the thermal noise, and at which $I \ll[G /(\partial R / \partial T)]^{1 / 2}$. Thus self-heating effects are negligible, and $G_{e} \approx G$.

Finally we consider the contribution of the preamplifier noise. At frequencies above the $1 /$ f noise region, the rms noise of our preamplifier with a source impedance of $10^{5} \Omega$ is about $2 \mathrm{nVHz}^{-1 / 2}$. This value corresponds to an rms voltage $V_{T}$ of about $6 \times 10^{-12} \mathrm{VHz}^{-1 / 2}$ referred to the input of the transformer (the cooled transformer does not contribute significantly to the preamplifier noise). Equating $\sqrt{2} V_{T}$ (the factor of $\sqrt{2}$ arises from the demodulation scheme) to $\mathrm{V}$ 
in Eq. (4.1), we find

$$
(N E P)_{A}=V_{T} G_{e} / I(\partial R / \partial T)
$$

Choosing $I=2 \mu \mathrm{A}$, we find $(\mathrm{NEP})_{\mathrm{A}} \approx 2 \times 10^{-16} \mathrm{WHz}^{-1 / 2}$, a value that is well below (NEP) Th $^{\circ}$

Provided that the I/f noise in the Al film is reduced sufficiently by strong coupling of the film to the substxate, it is evident that the electrical NEP should be Iimited by the intrinsic themal fluctuations of the bolomter for the values of the parameters we have chosen. It is, of course, necessary to make the contributions of the miscellaneous noise sources negligible. It seems impractical to make reliable a priori estimates of these contributions.

\section{B. EXPERIMENTAL MEASUREMENTS}

The values of $G, \tau$, and $C$ were estimated with the feedback circuit disconnected and with $I \ll[G /(\partial R / \partial T)]$, so that $G_{e} \approx G$. First, we determined the resistance of the Al strip as a function of temperature. Second, the value of $G$ was found by dissipating a known amount of power in the $B i$ heater and measuring the change in temperature. Third, an ac current of irequency $(\omega / 2)$ was applied to the $B i$ heater, so that the bolometer temperature oscillated at frequency $\omega$. By measuring the response of the bolometer as a function of frequency, we determined $\tau$, and hence $C=T G$. The responsivity was then determined with the bolometer in the feedback mode. A current at a low frequency $\omega / 2$ was passed chrough the $B 1$ heater, and the output of the closed loop was lock-in detected. From this measurement we determined the responsivity 
of the bolometer referred to the output of the cooled transformer. The spectral density of the voltage nolse at the output of the closed 100 was measured using an on-1ine PDP-11 computer, ${ }^{22}$ and its value, referred to the output of the transformer, was calculated. From these two measurements the NEP was detemined.

We evaluated the performance of five bolometers in some detail. Their important parameters are listed in Table II. The large range of values of $\mathrm{dR} / \mathrm{d} \mathrm{T}$ is evident. (Notice that the Al films on bolometers 1 and 2 did not have cut edges.) However, good values of the NEP could be obtained in each case by adjusting the bias current so that IdR/dT had the same value. Large bias currents are undesirable for low background operation because the high dissipation produced raises the temperature of the bolometer significantly above the bath temperature. Consequently, values of $\mathrm{dR} / \mathrm{dT}$ below about $100 \Omega \mathrm{K}^{-1}$ were considered too low for practical bolometers. When the edges of the Al film were trimmed, values of $\mathrm{dR} / \mathrm{dT}$ of $200 \mathrm{OK}^{-1}$ or greater were always obtained. The bias current listed represent minimum values; higher values (up to a factor of 10 higher for the smaller bias currents) produced essentially the same value of NEP at fxequencies near $5 \mathrm{~Hz}$. This result indicates that the NEP was limited by thermal noise and/or $1 / f$ nolse in the film, rather than by Johnson noise or preamplifier noise.

The parameters for bolometer 5 are given in greater detail in Tables III and IV. Table III lists the calculated heat capacities of the components of bolometer 5, and Table IV 11sts its relevant measured electrical and thermal parameters. The measured heat capacity, $1.2 \times 10^{-9} \mathrm{JK}^{-1}$, is in good agreement with the calculated value, 
$1.15 \times 10^{-9} \mathrm{JK}^{-1}$. In Fig. 5 the dashed line represents the responsivity referred to the output of the bridge calculated from Eqs. (3.1) and (4.1) using the appropriate parameters from Table IV. The roll-off below $1 \mathrm{~Hz}$ is due to the effect of the feedback system, and the rol1-off above $1 \mathrm{~Hz}$ is due to the time constant of the bolometer. The dots are the measured responsivity, and are in excellent agreement with the calculated values. In Fig. 6, the dashed line is the sum of the thermal noise, Johnson noise, preamplifier noise, and bath noise power spectra referred to the bridge with the bolometer in the feedback mode. A power spectrum of $1.6 \times 10^{-12}\left(1 \mathrm{~Hz}^{2} / \mathrm{f}^{2}\right) \mathrm{K}^{2} \mathrm{~Hz}^{-1}$ has been fitted to the data for the helium bath temperature fluctuations. Above $1 \mathrm{~Hz}$, it is evident that the measured noise power spectrum is about a factor two greater than the calculated noise. It is likely that the excess noise arises from the intrinsic "1/f noise" of the Al film, which is expected to have a white power spectrum in this frequency range (see Section $A$ ). The excess noise in the range 0.1 to $1 \mathrm{~Hz}$ probably arises from an instability In the feedback system. Fig. 7 shows the calculated and measured NEPs, obtained by taking the square root of the ratio of the data in Figs. 5 and 6. Above about $1 \mathrm{~Hz}$, the measured NEP is somewhat higher than the calculated NEP. For example, at $2 \mathrm{~Hz}$ the measured NEP is about $1.7 \times 10^{-15} \mathrm{WHz}^{-1 / 2}$, compared with a thermal noise value of $1.3 \times 10^{-15} \mathrm{WHz}^{-1 / 2}$. As the frequency increases, the NEP slowly degrades, for example, to $2.0 \times 10^{-15} \mathrm{WHz}^{-1 / 2}$ at $5 \mathrm{~Hz}$. This degradation reflects the fact that the responsivity falls as the frequency increases, while the Johnson noise and preamplifier noise remain constant. A slightly improved responsivity could probably have been 
obtained at the higher frequencies by operating the bolometer with a higher bias current. However, the thermal feedback would then be higher, and the bolometer nearer to thermal runaway.

The dynamic range of the bolometer is approximately $\delta \mathrm{TG} /(\mathrm{NEP})$, where $\delta T$ is the half-width of the transition and (NEP)/G is the temperature fluctuation per $\mathrm{Hz}^{1 / 2}$. Taking $\delta \mathrm{T} \sim 2 \mathrm{mK}$ and $(\mathrm{NEP}) / \mathrm{G} \sim 10^{-7} \mathrm{KHz}^{-1 / 2}$, we find a dynamic range of about $10^{4}$ in a $1 \mathrm{~Hz}$ bandwidth. The maximum long-term change in temperature (due to a change in the background radiation or a change in the temperature of the helium bath) that can be cancelled by the feedback system is $\sim \alpha \delta \mathrm{T} \sim 0.1 \mathrm{~K}$. This value corresponds to a change in background radiation of about $1 \mathrm{nW}$ 
V. FAR INERARED ABSORBER

\section{A. THEORY}

The portions of the bolometer that can absorb a significant amount of infraxed radiation axe the dielectric substrate and the conducting film Both sapphire and (most types of) diamond have absorption bands in the near infrared. At far infrared wavelengths where these composite structures are expected to be most useful, however, the cold substrate acts as an essentially lossless dielectric with an index of refraction which is only weakly dependent on frequency. The substrate serves to impedance match free space radiation into a thin metal film deposited on the back side of the bolometer. The surface impedance of the film is selected for optimum absorption. We calculate the absorptivity under the following assumptions: The absorbing layers comprise an infinite plane; the sapphire has a thickness d, a refractive index $n$ (we neglect birefringence), and a permeability equal to that of free space; the conducting layer has an infrared frequency resistivity per square, $R_{\square}$, that is the same as at zero Erequency, and a thickness that is much smaller than the skin depth and the wavelength of the radiation; the radiation is unpolarized; the radiation makes only a single pass at the bolometer (i.e. there is no reflecting surface under the bolometer); and the radiation is incident normally on the surfaces.

The transmission and reflection coefficients at the upper (uncoated surface) are 23

$$
T_{1}=4 n /(n+1)^{2}
$$


and

$$
R_{1}=(n-1)^{2} /(n+1)^{2}
$$

The transmission, reflection, and absorption coefficients at the lower (coated) surface are

$$
\begin{gathered}
T_{2}=4 n /\left(n+1+z_{0} / R_{D}\right)^{2} \\
R_{2}=\left(1-n+z_{0} / R_{\square}\right)^{2} /\left(n+1+z_{0} / R_{0}\right)^{2}
\end{gathered}
$$

and

$$
A_{2}=\left(4 n z_{0} / R_{0}\right) /\left(n+1+z_{0} / R_{0}\right)^{2}
$$

where $Z_{0}(\approx 377 \Omega / \square)$ is the impedance of free space. Although Eqs. (5.1) to (5.5) are derived for normal incidence, they remain accurate to within a few percent for angles of incidence up to $60^{\circ}$. In general, there will be multiple reflections between the two surfaces of the substrate, giving rise to Fabry-perot fringes in the observed values of the transmissivity, $T_{b}$, reflectivity, $R_{b}$, and absorptivity, $A_{b}$, of the bolometer. It can readily be shown that

$$
\begin{aligned}
& T_{b}=\frac{T_{1} T_{S} T_{2}}{1+T_{S}^{2} R_{1} R_{2} \pm 2 T_{S} \sqrt{R_{1} R_{2}} \cos \delta}, \\
& R_{b}=\frac{R_{1}+T_{S}^{2} R_{2}-2 T S \sqrt{R_{1} R_{2}} \cos \delta}{1+T R_{1}^{2} R_{1} R_{2} \pm 2 T_{S} \sqrt{R_{1} R_{2}} \cos \delta} \\
& \text { and } \\
& A_{b}=\frac{T_{1}\left(1-T_{S} T_{2}-T R_{2}\right)}{1+T_{S}^{2} R_{1} R_{2} \pm 2 T_{S} \sqrt{R_{1} R_{2}} \cos \delta} \text {. }
\end{aligned}
$$

The plus sign applies when $z_{0} / R_{0}>n-1$, and the minus sign applies 
when $Z_{0} / R_{D}<n-1$. In these equation, $T_{S}$ is the transmissivity of a thickness d of sapphire, $\delta=4 \pi n d \bar{v}$, and $\bar{v}$ is the free-space wavenumber of the radiation. In practice, all three expressions will be complicated by the fact that sapphire is birefringent.

A particular simple case arises when the reflection from the metal film $R_{2}=0$, that is when $Z_{0} / R_{\square}=n-1$ [from Eq. (5.4)]. There are no multiple reflections within the sapphire, and the absorptivity of the bolometer is Independent of frequency. With $\mathrm{T}_{\mathrm{S}}=1$, we find

$$
A_{b}\left(Z_{0} / R_{\square}=n-1\right)=4(n-1) /(n+1)^{2} .
$$

If we take $n \approx 3$, as is appropriate for sapphire, we find $A_{b}\left(z_{0} / R_{\square}=n-1\right) \approx 0.5$. Any other value of $n$ produces a smaller value of the frequency-independent absorptivity. If the bolometer is to be used over a wide range of frequencies, it is usually desirable to choose $R_{Q}=z_{0} /(n-1) \approx z_{0} / 2$, so that the absorption is frequency-independent. On the other hand, if the bolometer is to be used over a relatively narrow frequency band, one can take advantage of the Fabry-Perot fxinges to obtain a higher absorptivity. As an example, take $\delta=2 \pi$, corresponding to the case $\bar{\nu} \approx 1 / 2 \mathrm{nd}$, and $T_{S}=1$. If $d=0.05 \mathrm{~mm}$, and $\mathrm{n}=3$, the absorptivity has a peak at about $33 \mathrm{~cm}^{-1}$, and has minima at about $17 \mathrm{~cm}^{-1}$ and $50 \mathrm{~cm}^{-1}$. The peak absorptivity is found by setting $\cos \delta=1$ in $E q$. (5.8), and maximizing $A_{b}$ with respect to $Z_{0} / R_{0}$ (with $T_{S}=1$ ). If we assume $n=3$, we find that the maximum value of $A_{b}$ is 0.90 , with $Z_{0} / R_{\square}=10$. In practice, the Fabry-Pérot pattern for sapphire will be complicated by the presence of the extraordinary ray (for which $n \approx 3.3$ ), and will depend on the cut of the sapphire crystal. 
No such complications arise for diamond, which is optically isotropic.

\section{B. EXPERIMENTAL CHARACTERTZATTON OF COATED SUBSTRATES*}

Bismuth films varying in thickness from $30 \mathrm{~nm}$ to $480 \mathrm{~nm}$ were deposited on $8 \times 4 \times 0.135 \mathrm{~mm}$ sapphire substrates. Bismuth was used for the absorbing film because the required resistivity range could be achieved with a convenient range of thicknesses. Other materials, for example, chromium or nickel-chromium alloys, would presumably work equally well. These substrates are optically polished on one side and are rough on the reverse side. The rough side has irregularities large compared with the thickness of the films, but small compared with far infrared wavelengths. For each film thickness, a sample was prepared on both smooth and rough surfaces. The resistivities of these films measured at $300 \mathrm{~K}$ and $4.2 \mathrm{~K}$ are show in Fig. 8 . The resistivities at $300 \mathrm{~K}$ are greater than the bulk value and increase as the films are cooled to $4.2 \mathrm{~K}$. These results suggest that the structure of the films is somewhat non-crystalline. Nevertheless, the resistivities were quite reproducible, and the desired resistance could be obtained readily.

The far infrared transmission of the coated substrates was measured at $1.3 \mathrm{~K}$ using a Fourier-transform spectrometer and a germanium bolometer. Transmissivity spectra were obtained for an empty sample holder, for an uncoated substrate, and for four substrates with varying film thicknesses. Measurements were made over three overlapping spectral ranges, from 3 to 25,10 to 120 , and 15 to $300 \mathrm{~cm}^{-1}$ with resolutions

\footnotetext{
* Experiments in this section were done by G.I. Hoffer.
} 
of $0.25,1.2$, and $3.0 \mathrm{~cm}^{-1}$, respectively. Transmissivicy spectra were obtained by dividing the transmission spectrum of each sample by the transmission spectrum of the empty sample holder. A series of transmissivity spectra is shown in Fig. 9. The Fabry-Pérot fringes are very pronounced for the uncoated substrate, and have a period of about $12 \mathrm{~cm}^{-1}$ as expected for $d=0.135 \mathrm{~mm}$ and $\mathrm{n} \approx 3$. The beat pattern is due to the difference $\left({ }^{\wedge} 0.3\right)$ in the refractive indices for the ordinary and extraordinary rays. The coated substrates show reducttions in the overall transmission and in the amplitude of the Fabry-Pérot fringes. The latter effect is due to the reduced reflection at the back surface when it is coated with a conducting layer. According to Eq. (5.4), the reflectivity goes to zero (and hence the fringes vanish) when $R_{\square}=z_{0} /(n-1)\left(\approx z_{0} / 2 \approx 1888 / 0\right.$ for sapphire $)$. In Fig. 9, the minimum in the fringe amplitude occurs between $R_{\square}=149$ and $235 \Omega / \square$. This is consistent with the theoretical predictions.

\section{OPTICAL CALIBRATION OF INFRARED SOURCES}

A blackbody at fixed temperature $T$ is a standard infrared source. The emitred radiation can be calculated from Planck's formula. To conduct the radiation to the detector at the bottom of the dewar, we have to use optical components such as a light pipe, mitered right angles, or condensing cones. Appropriate fllters have to be inserted into the path to cut off the unnecessary radiation. All these elements need to be calibrated, but the difficulty is that none of these elements obeys a linear superposition law. By putting two of the elements in 
series, the total transmissivity is not necessarily the product of the individual transmissivities measured separately. For example, in a light pipe the rays which make a large angle $\theta$ with the axis of the light pipe will be bounced back and forth more times by the wall than those with a small angle $\theta$. Thus the rays with larger $\theta$ will be attenuated more. The relation between incident and transmitted power is

$$
\int W_{L}(\theta) d \theta=\int T(\theta) W_{i}(\theta) d \theta
$$

where $w_{i}(\theta)$ and $w_{t}(\theta)$ are the angular power distributions for incident and transmitted radiation, respectively. $T(\theta)$ is the angular transmissivity of the light pipe. If we measure the total incident and transmitted power, the ratio $\int w_{t}(\theta) d \theta / \int w_{j}(\theta) d \theta$ cannot be generalized as is apparent from Eq. (5.10). Theoretical calculations on the transmission of light pipe have been made by Richards 24 and Loewenstein. 25 The uncertainty in estimating the reflectance on the inner wall makes it difficult to apply to real situations.

For mitered right angles, the situation is even more complicated. The deflection of meridional rays (rays whose paths lie in the plane of symmetry of the pipe under consideration) is illustrated in Fig. 10 . The skew rays will be scattered more by the side walls. After passing through the mitered corner, the beam no longer has a well-defined solid angle distribution. This problem should be avolded in the infrared calibration. Other components such as incident cones and condensing cones also add confusion to the calibration, although they may increase the optical efficiency. 
To measure the optical absorptivity of the bolometer, we adopted the simplest design we could devise. Two polished aluminum mirrors and a light pipe were used to feed the radiation to the detector. The configuration is shown in Fig. 11. The incident radiation used in the calibration has solid angles less than $7 \times 10^{-3} \mathrm{sr}$. In this case the transmissivities of the various filters are independent of the solid angles. The overall transmissivity of the filters is the product of the transmissivities for each filter at a given wavelength.

\section{FAR INERARED EFTICIENCY OF BOLOMETER}

The far infrared absorptivity of the bolometer was measured by chopping the radiation falling on the top of the light pipe between sources at $77 \mathrm{~K}$ and $290 \mathrm{~K}$. The transmissivity of the light pipe and of the various elements in the light pipe were measured separately, so that the power incident on the bolometer could be computed. The ratio of the absorbed power to the incident power was the absorptivity.

The cold source was contalned in a $150 \mathrm{~mm}$ dlameter glass dewar filled with liquid nitrogen. The lower part of the inside of the dewar was covered with $A N-72$ EccosorbTM, and the upper part was covered with aluminum sheet which was in contact with the liquid nitrogen. Dry nitrogen gas was flushed over the top of the dewar to avold condensation of moisture on the inner wall. The cold radiation traveling upward fron the dewar was deflected downward into the light pipe by two polished aluminum mirrors. A circular aperture $29 \mathrm{~mm}$ in diameter limited the solid angle viewed by the light plpe. The transverse dimension of the aluminum mirrors is much larger than the 
aperture. The geometrical circle on the mirror seen by the bolometer with an angular field of view less than $7 \times 10^{-3} \mathrm{sr}$ has a diameter not more than $5 \mathrm{~cm}$, while the aluminum mirror is $15 \mathrm{~cm} \times 20 \mathrm{~cm}$. The diffraction of the room temperature radiation by the mixror edge into the bolometer is negligible. The separation between the aperture and the light pipe could be varied to change this solid angle. An $11 \mathrm{~Hz}$ reflecting chopper with its blades at $45^{\circ}$ to the horizontal was located below the aperture so that the signal reaching the light pipe arrived alternately from the aperture and from an ambient temperature slate blackboard. The slate was a convenient reference because of its high absorptivity and long thermal time constant. The calibration was accomplished by measuring the difference between the lock-in detected signal from the cold source with the aperture open and the signal measured when an ambient temperature $(290 \mathrm{~K})$ piece of Eccosorb was placed on top of the horizontal metal plate which contained the aperture.

The stainless steel light pipe (760 mm long and $11.7 \mathrm{~mm} \mathrm{i.d.)} \mathrm{was}$ sealed at the top with a $0.2 \mathrm{~mm}$ thick black polyethylene sheet. The vacuum can at the bottom was sealed with a wedge-shaped sapphire window that tapered in thickness from $1.52 \mathrm{~mm}$ to $1.02 \mathrm{~mm}$. A $2.54 \mathrm{~mm}$ thick Fluorogold ${ }^{T M}$ low-pass filter was placed in the liquid helium above the sapphire window to define the spectral bandpass and to reduce the background loading on the bolometer. The lower side of the bolometer substrate $(4 \times 4 \times 0.05 \mathrm{~mm})$ was coated with a $80 \mathrm{~nm}$ bismuth film with a resistance of approximately $190 \mathrm{~S} / \mathrm{D}$ at $1.5 \mathrm{~K}$. Thus the absorption was approximately independent of frequency. A slab of Eccosorb TM was 
placed behind the bolometer to absorb the radiation which by-passed the bolometer or was transmitted through it. Consequently, the radiation made only a single pass at the bolometer.

The transmissivity of the FluorogoldTM filter at $1.5 \mathrm{~K}$ was measured using a Fourier transform spectrometer, and is plotted in Fig. 12(a). Figure 12(b) shows the calculated difference between the blackbody sources at $290 \mathrm{~K}$ and $77 \mathrm{~K}$ as viewed through the FluorogoldTM filter. This curve represents the $\sim 5$ to $45 \mathrm{~cm}^{-1}$ pass-band of the calibration. We estimate an exror of $\pm 3 \%$ in the power calibration.

The transmission of the light pipe was measured as shown in Fig. 13. The detector used in this experiment was an InSb detector. A black polyethylene sheet and a Fluorogold TM filter constituted the window of the detector. The Fourier spectrum of the mercury arc using this detector showed a passband between approximately 5 and $45 \mathrm{~cm}^{-1}$. A piece of brass light pipe about $50 \mathrm{~mm}$ long and $11.7 \mathrm{~mm}$ i.d. was connected to the detector input. The light pipe could be evacuated through a small tube in the wall. A $0.2 \mathrm{~mm}$ thick mylar sheet sealed the front end. The transmissivity of the brass light pipe was over 0.98 (much higher than that of the S.S. 1ight pipe to be measured). The chopper again chopped the blackbody radiation between $77 \mathrm{~K}$ and $290 \mathrm{~K}$. The responses of the detector at both position I and II were measured. At position II the mylar sheet was moved to the front of the S.S. Iight pipe so that the whole light pipe was evacuated. The solid angle of the input radiation could be changed by varying the distance between position I and the aperture. The measured transmissivity was $0.61 \pm 0.03$ independent of the solid angles $\left(<7 \times 10^{-3} \mathrm{sr}\right)$. The light pipe was also cooled 
down to liquid nitrogen temperature by enclosing the light pipe with a plastic bag and feeding liquid nitrogen into it. The front end of the light pipe was kept warm to avoid the condensation of water vapor on the mylar window. The measured transmissivity was unchanged to within $2 \%$. This value for $\mathrm{T}_{1 \mathrm{p}}$ can be brought into agreement with the calculated transmissivity from a light pipe theory which ignores paraxial rays, 24 if we assume a resistivity which is three times largex than the measured low temperature value of approximately $70 \mu \Omega \mathrm{cm}$. Other experimenters have found a similar discrepancy. 24

The transmissivity of the sapphire window at $1.5 \mathrm{~K}$ was measured to be $0.57 \pm 0.02$. This value is in excellent agreement with the calculated value of 0.57 obtained assuming refractive indices of 3.0 and 3.4 and assuming negligible absorption. The transmissivity of the black polyethylene window at room temperature was measured to be $0.86 \pm 0.02$. The absorbing area of the bolometer was reduced from $16 \mathrm{~mm}^{2}$ to $A_{e f f}=14.6 \mathrm{~mm}^{2}$ by the superconducting In and Al films, which have negligible absorption in the far infrared. We assume that the fraction of the radiation leaving the light pipe which is incident on the detector is equal to the ratio of $\mathrm{A}_{\text {eff }}$ to the area of the light pipe.

The far infrared responsivity of the bolometer was measured over a range of solid angles from 2.0 to $6.9 \times 10^{-3} \mathrm{sr}$. As expected, the data show no significant dependence on solid angle. The average value of the absorptivity is $0.52 \pm 0.05$ (see Fig. 14). The aggregate error limit of \pm 0.05 is obtained by combining errors of $\pm 5 \%$ in the measurements of the electrical NEP and the far infrared responsivity with 
values given above for the errors in the measurements of the transmissivity of the black polyethylene, the light pipe, the FluorogoldT, and the sapphire. This value is in excellent agreement with the theoretical value of 0.5 . The absorptivity is expected to be the same up to the plasma frequency of $B 1^{26}\left(\approx 160 \mathrm{~cm}^{-1}\right)$ where the resistivity will deviate from the dc value.

The effective absorptivity, $\varepsilon_{e}$ (relative to the area of the entire bolometer), is $0.47 \pm 0.05$. Using our best value of electrical NEP, $(1.7 \pm 0.1) \times 10^{-15} \mathrm{WHz}^{-1 / 2}$, we find $D^{*}=\varepsilon \mathrm{e}^{\mathrm{A}^{1 / 2} / \mathrm{NEP}}=(1.1 \pm 0.1) \times 10^{14} \mathrm{~cm} \mathrm{~W}^{-1} \mathrm{~Hz}^{1 / 2}$. We also tried to measure the optical efficiency of the bolometer in the absence of the light pipe. But, unfortunately, the uncertainty in the scattering of the diffracted radiation by the wall of the dewar, which was covered with Eccosorb ${ }^{T M}$, made the calibration very confusing. 


\section{POSSIBLE IMPROVEMENTS}

Some improvements in the sensitivity of the transition edge bolometer appear possible. The heat capacity of the bolometer could be reduced by eliminating the bismuth heater and using only two indium contacts with much smaller areas. The substrate could be supported by uncoated nylon threads of negligible thermal inductance. By means of these modifications, the heat capacity would be just that of the substrate and the absorbing film. Table 5 shows the heat capacity of several materials. At $1.27 \mathrm{~K}$, a diamond substrate and chromium absorber would give a heat capacity of $1.2 \times 10^{-10} \mathrm{JK}^{-1}$, about one oxder of magnitude smaller than the present bolometer. For the same value of thermal conductance, the time constant of the bolometer would be reduced to about $6 \mathrm{~ms}$. Alternatively, the thermal conductance could be reduced to about $2 \times 10^{-9} \mathrm{WK}^{-1}$, keeping the time constant at its present value, $60 \mathrm{~ms}$. Provided that the bolometer was still essentially thermal noise limited, an improvement in the NEP by a factor of $\sqrt{10}$ could be achieved.

A further improvement in sensitivity could be obtained by cooling the bolometer to liquid ${ }^{3}$ He temperature, and operating at about $0.4 \mathrm{~K}$ using a titanium film as the thermometer. We estimate that a bolometer using a diamond substrate and bismuth absorber would have a heat capacity of $8 \times 10^{-12} \mathrm{JK}^{-1}$. The thermal conductance could be reduced to $1.3 \times 10^{-10} w^{-1}$ for a time constant $60 \mathrm{~ms}$. The thermal noise limited NEP $\left(\alpha \mathrm{TG}^{1 / 2}\right)$ would then approach $3 \times 10^{-17}$. WHz ${ }^{-1 / 2}$. In order to achieve this limit, a preamplifier with a nolse temperature of less than $0.4 \mathrm{~K}$ would be required. 
The optical efficiency of the bolometer could be increased by the use of focusing and partially recollimating cones. The analysis shows the absorptivity of the bolometer is essentially a constant up to $60^{\circ}$ from normal incidence. An optimized design could easily be obtained by recollimating the incident radiation to within $60^{\circ}$. We could even put the bolometer into an integrating cavity so that the radiation would make multiple passes through the absorbing area. For low frequency applications the noise from He bath fluctuation could be reduced greatly by inserting a second thermal sink between the copper block and the He bath. The temperature of the second thermal sink would be regulated by a separate thermometer and a heater. The low frequency roll-off knee in the bolometer feedback circuit could then be shifted to a much lower frequency to obtain a wider region of flat response. 
VIT. THEORY OF THERMAL FLUCTUATION NOISE IN METAL THIN FILMS

\section{A. INTRODUCTION}

Clarke and Voss ${ }^{22}$ have developed a model that used equilibrium temperature fluctuations to predict the voltage noise observed at room temperature in metal thin films deposited on glass substrates. The temperature fluctuations produce resistance fluctuations through the temperature coefficient of resistance, $\beta=(1 / R)(d R / d T)$. When a constant dc bias current is applied to the thin film, the resistance fluctuations give rise to voltage fluctuations. In the frequency range from $0.1 \mathrm{~Hz}$ to $1000 \mathrm{~Hz}$, this model predicts a $1 /$ f noise power spectrum which quantitatively agrees with the experimental measurements.

Subsequently, Clarke and $\mathrm{Hsiang}^{3}$ measured the noise power spectrum of $\mathrm{Sn}$ and $\mathrm{Pb}$ thin films in the middle of the superconducting transition. They found $1 /$ f noise when the film was poorly coupled to the substrate (Type A). The noise level was again consistent with the equilibrium temperature fluctuation model. But, when the film was strongly coupled to the substrate (Type B), the low frequency noise tended to be flattened off. Due to the complication of the boundary impedance and the mismatch of thermal phonons at the metal-insulator interface, the clarke-Voss model could not give any quantitative predictions for type B samples. Ketchen and Clarke ${ }^{27}$ measured the noise from freely suspended Sn films at the superconducting transition. They had a well-defined 1dimensional system with no substrate. Each film was freely suspended between two pairs of clamps that thermally grounded the ends of the film. The boundary conditions could be easily applied. The equilibrium 
temperature fluctuation model predicted a white nolse reglon followed by a roll-off above a low frequency knee. This was in good agreement with the nolse observed in their experiments.

The equilibrium temperature fluctuation model was successful in predicting the $1 / 5$ noise at room temperature and the noise at the superconducting transition. However, recent experiments ${ }^{28}$ showed that the $1 /$ f noise in films on substrates near room temperature had a stronger temperature dependence than the model predicted.

\section{B. EQUILIBRIUM TEMPERATURE FLUCTUATIONS}

In a homogeneous medium with specific heat $C_{v}$ and thermal diffusivity $D$, the temperature $T(\vec{x}, t)$ obeys the diffusion equation

$$
\frac{\partial T}{\partial t}=D \nabla^{2} T
$$

A random driving term can be added to $\mathrm{Eq}$. (7.1) to generate noise. The actual temperature fluctuation is governed by the diffusion equation and the corresponding boundary conditions. Clarke and Voss ${ }^{22}$ suggested two different kinds of random driving terms. When added to the diffusion equation, they become

$$
\begin{aligned}
& \frac{\partial T}{\partial t}=D \nabla^{2} T+C_{v}^{-1} \nabla \cdot \vec{F}(\vec{x} \cdot t) \\
& \frac{\partial T}{\partial t}=D \nabla^{2} T+C_{v}^{-1} P(\vec{x} \cdot t)
\end{aligned}
$$

where $\overrightarrow{\vec{F}}(\overrightarrow{\mathrm{x}}, t)$ and $P(\vec{x}, t)$ obey the relation

$$
\langle\vec{F}(\vec{x}, t) \cdot \vec{F}(\vec{x}+\vec{s}, t+\tau)\rangle=(2 \pi)^{3} F_{0}^{2} \delta(\vec{s}) \delta(\tau),
$$

and

$$
\langle\mathrm{P}(\vec{x}, t) \cdot \overrightarrow{\mathrm{P}}(\overrightarrow{\mathrm{x}}+\overrightarrow{\mathrm{s}}, t+\tau)\rangle=(2 \pi)^{3} \mathrm{p}_{\mathrm{o}}^{2} \delta(\vec{s}) \delta(\tau) .
$$


In $\mathrm{Eq} \cdot(7,2), \overrightarrow{\mathrm{F}}(\mathrm{x}, \mathrm{t})$ represents a random themal current flowing inside the system. The randon current is uncorrelated in space or time. The space-time correlation function of the temperature fluctuation $C_{\mathrm{T}}(\overrightarrow{\mathrm{s}}, \tau) \equiv\langle\Delta \mathrm{T}(\overrightarrow{\mathrm{x}}+\overrightarrow{\mathrm{s}}, \tau+\tau) \Delta \mathrm{T}(\overrightarrow{\mathrm{x}}, t)\rangle$, is given by

$$
C_{T}(\vec{s}, \tau)=\left[k_{B} T^{2} / C_{v}(4 \pi D \tau)^{1 / 2}\right] \exp \left(-s^{2} / 4 D \tau\right)
$$

It can be seen that the temperature fluctuation is spatially uncorrelated as $\tau+0$. Therefore, this model is called the uncorrelated model.

The second kind of dxiving term $P(\vec{x}, t)$ in $E q .(7,3)$ represents a random thermal source or sink, which is still spatially and cemporally uncorrelated. Detailed calculations show a correlation function

$$
C_{T}(\vec{s}, w)=\frac{p_{0}^{2} e^{-|\vec{s}| / \lambda}}{8 \sqrt{2} \pi^{2} D^{3 / 2} w_{w}^{1 / 2}} \frac{\sin (|\vec{s}| / \lambda)}{|\vec{s}| / \lambda},
$$

where $\lambda(w)=(2 \mathrm{D} / \mathrm{w})^{1 / 2}$. For a particular harmonic component in the fluctuation with frequency $w$, there is a spatial region with Iinear dimension $\lambda(\mathrm{s})$ which has correlated temperature fluctuations. This model is called the correlated model.

For a homogeneous medium, the temperature fluctuation of a small box with dimensions $\ell_{1} \times l_{2} \times \ell_{3}$ can be analyzed. The total temperature fluctuation should be normalized to $\mathrm{kT}^{2} / \mathrm{C}_{\mathrm{v}}$. For the uncorrelated mode1, the predicted spectrum shows four frequency regions with Exequency dependence given by $f^{0}, \ln (1 / \xi), f^{-1 / 2}$, and $f^{-3 / 2}$, respectively. There are three knees separating these regions. The knee frequencies are $f_{1}=0 / 4 \pi l_{1}^{2}(1=1,2,3)$. The correlated model has four Exequencyodependent regions given by $f^{-1 / 2}, f^{-1}, f^{-3 / 2}$, and 
$f^{-2}$. The knees still appear at the same frequencles as the uncorrelated model. All these analyses become wuch more complicated in a composite system made up of a thin film on the substrate. Though the noise spectrum cannot be analyzed explicity, it is still possible to find out the nature of the random driving term.

\section{AUTOCORRELATION FUNCTION AND DECAY MEASUREMENTS}

According to the Wiener-Khintchine relations, the noise power spectrum $S(w)$ of a stochastic process $A(t)$ can be related to the autocorrelation function $C(\tau)$ :

$$
S(w)=(2 \pi)^{-1} \int_{0}^{\infty} C(\tau) \cos w \tau d \tau
$$

and

$$
C(\tau)=\langle A(t) A(t+\tau)\rangle
$$

The total noise power is the integral of the spectrum over the whole frequency range, that comes out to be $\mathrm{C}(0)$. Therefore, if we can find the autocorrelation function and properly normalize the first point to the total noise power, the noise power spectrum can be calculated from Eq. (7.8). Since the equilibrium temperature fluctuations on the average obey the same decay law as small macroscopic perturbations of the system, the autocorrelation function of temperature fluctuations can be generated by uniformly perturbing the system and looking at the decay of the average temperature in the system. It can be shown ${ }^{22}$ that the autocorrelation function for the uncorrelated model corresponds to the $\delta$-function decay curve, that is, the thermal decay curve in time generated by a spatially uniform and short-duration heat pulse. On the other hand, the 
autocorrelation function for the correlated model corresponds to the step-function decay curve, which is the integral of the $\delta$-function decay. The cosine transform of these decay curves can be compared with the measured noise power spectrum. The importance of this result is that it gives an experimental method of determining the shape of the noise power spectrum in an arbitrary volume with arbitrary coupling once the nature of the random noise generating term is known.

At room temperature, the composite system made up of a metal thin film on a glass substrate shows $1 /$ f noise from $0.1 \mathrm{~Hz}$ to $1000 \mathrm{~Hz}$. The spectrum obtained from the cosine transform of a step-function decay has the same frequency dependence. Therefore, the correlated model is more appropriate in describing this system. For a freely suspended Sn fiber at the superconducting transition, the spectrum from the cosine transform of the $\delta$-function response is in excellent agreement with the measured noise power spectrum. The uncorrelated model should be used to describe the freely suspended fiber at the superconducting transition. It will be interesting and important to find out the random noise generating term for a Sn film on a substrate at the superconducting transition: Whether the correlated model or the uncorrelated model more accurately describes this particular system. We used a SQUID 29 (Superconducting QUantum Interference Device) voltmeter to measure the noise from $\mathrm{Sn}$ films evaporated on sapphire substrates. The measured noise power spectrum was compared with the cosine transforms of both f-function and step-function decay curves. We found that the uncorrelated model was the better choice. 
VIII. EXPERIMENTAL PROCEDURES

\section{A. SAMPLE PREPARATION}

Single crystal sapphixe substrates with dimensions $8 \mathrm{~mm} \times 4 \mathrm{~mm} \times 0.135 \mathrm{~mm}$ were used in the experiment. One surface of the substrate was polished for film deposition. The substrate was first cleaned with Labtone and then degreased in trichloroethylene and methanol. Finally it was rinsed with distilled water and blown dry with pressurized nitrogen gas. A Sn film about $1000 \AA$ thick was evaporated on the substrate at room temperature at a pressure of approximately $3 \times 10^{-5}$ torr. The film was then cut with a diamond knife into the shape shown in Fig. 15. The central region with dimensions $500 \mu \times 12 \mu \times 1000 \AA$ is the sample for the experiment. Four indium contacts were pressed onto the film For external electrical connections.

The slowly evaporated tin film $(7 \AA / \mathrm{sec})$ and the rapidly evaporated tin film ( $100 \mathrm{~A} / \mathrm{sec})$ look quite different with naked eyes. The latter is highly reflecting while the former is not.

The scanning electronmicroscope studies showed different structures. Fig. 16a is a picture of a slowly evaporated film. There are many submicron grains. For a rapidly evaporated film, no visible grain is observed with a magnification of $10^{4}$ (see Fig. 16b). The relation between the grain size and the evaporation rate can be understood from the processes in the formation of the thin films.

The condensation of a vapor atom ${ }^{30}$ is determined by the interaction with the surface of the substrate. Once the atom is adsorbed to the surface, it may or may not be in thermal equilibrium. The adatoms 
move over the surface by thermal excitation or their own kinetic energy parallel to the surface until chemically adsorbed onto the surface to form a stable cluster. These clusters constitute the nucleation centers and then grow to form islands. As islands increase their size by further deposition and come closer to each other, the larger ones appear to grow by coalescence of the smaller ones. The tendency to form larger islands is the result of higher surface mobility of the adatoms and clusters. Higher mobility is obtained 31,32 for films of low-melting-point materials, smooth substrate surface, low deposition rate, and high substrate temperature. The single-crystal substrate has a dominant influence on the oriented growth of the thin film. Epitaxy can occur between two substances of completely different crystal structures and of different types of chemical bonds. ${ }^{33}$ There exists a critical temperature, ${ }^{34}$ called the epitaxial temperature, above which epitaxy is perfect and below which it is imperfect. The epitaxial temperature is usually above room temperature. Even above the epitaxial temperature, the examination of the epitaxial thin film may reveal a variety of dislocation lines, stacking faults, grain boundaries, and minor defects from aggregation of point defects. 35

Due to the low melting point of tin ( $=505 \mathrm{~K})$, the adsorbed tin atoms have a fairly high surface mobility. The fine grains in fig. $16 \mathrm{~b}$ are due to the high deposition rate, which results in strong interactions of the adatoms with each other. They are, therefore, chemisorbed with little suxface migration. The sharp corners of the grains In Fig. 16a may be attributed to the imperfect epitaxial growth of the film. These explanations are further verified by using glass substrate 
instead of sapphire. Fig. 17a and 17b show the electronmicroscope pictures of slowly $(7 \AA / \mathrm{sec})$ and rapidly $(100 \AA / \mathrm{sec})$ evaporated tin films on glass substrate. A higher deposition rate still results in a smaller grain size. In the absence of epitaxial growth, the grains in Fig. 17 do not have sharp corners. For the same deposition rate (100 $\mathrm{A} / \mathrm{sec}$ ), the grains on sapphire substrate (Fig. 16b) are much finer than those on glass substrate (Fig. 17b). This might be due to the difference in the thermal conductivity of the substrate. At room temperature, the thermal conductivity of sapphire is about one order of magnitude higher than that of glass. The effective heating of the sapphire substrate by the thermal radiation from the evaporation boat is less, in which case the actual temperature of the substrate during the evaporation is lower.

In spite of the difference in microstructure for rapidly and slowly evaporated tin films on sapphire substrate, we still observed the same noise power spectrum and the same responses to $\delta$-function and step-function perturbations.

\section{B. SQUID ELECTRONICS AND CRYOSTAT}

We measured the noise from the tin sample by four terminal method to eliminate contact noises. A constant dc current was applied to the tin film. The SQUID circuit was designed as a nearly infinite input impedance voltmeter to read out the voltage across the tin film. The circuit diagram is shown in Fig. 18. $I_{1}$ is the bias current for the sample. $I_{2}$ is a second current source to maintain zero cixculating current around the loop $R-R_{S T D}-L_{i}$. When this condition is satisfied, 
then

$$
I_{1} R=I_{2} R_{S T D}
$$

The resistance of the sample can be calculated immediately. The SQUID signal was fed back to the standard resistor so that the SQUID served as a null detector. Any small change in the sample resistance would show up in the feedback current. From simple circuit theory, it can be shown that the overa11 voltage gain in the SQUID system is $R_{f} / R_{S T D}$. The voltage fluctuation across the feedback resistor $R_{f}$ is related to the temperature fluctuation of the sample by:

$$
\Delta V_{f}^{2}=\left(\frac{R_{f}}{R_{S T D}}\right)^{2} I_{1}^{2}\left(\frac{d R}{d T}\right)^{2} \Delta T^{2}
$$

In our experiment, we used a standard resistor $R_{\mathrm{STD}}=1.35 \Omega$ The input coil had 85 turns with a measured mutual inductance of $40 \mathrm{nH}$. Mercury cells were used in the current sources and ten-turn wire wound potentiometers were needed to vary the current from 0 to $20 \mu \mathrm{A}$. The tin samples with dimensions $500 \mu \mathrm{m} \times 12 \mu \mathrm{m} \times 1000 \AA$ usually had a room temperature resistance approximately $50 \Omega$. At $3.8 \mathrm{~K}$, just above the superconducting transition, the resistance was about 2.558 . A resistance ratio of 20 was observed.

The frequency response of the SQUID system depends on the standard resistor $R_{S T D}$ and the sample resistance $R_{\text {. In the circuit analysis }}$ in this section, we have assumed zero impedance for the input coil $L_{1}$. This is true for a frequency well below the characteristic ixequency of the 1oop $R-R_{S T D}-L_{i}$, which is given by $\left(R+R_{S T D}\right) /\left(2 \pi L_{1}\right) \approx 10^{5} \mathrm{~Hz}$. The intrinsic noise calculations of the SQUTD system are made more complicated 
by the interactions between the modulation coil, input coil, and the SQUID. A numerical calculation can be found in the work by Clarke and Tesche. 36

A schematic diagram of the SQUID insert is shown in Fig. 19. The SQUID system and the sample are mounted inside a vacuum can. The SQUID and the standard resistor are on copper block 1 , which has a good thermal coupling to the $\mathrm{He}^{4}$ bath. The sample is on copper block 2, which is supported by two thin wall stainless steel tubes to block 1. A thin copper wire links the two blocks so that the measured time constant for block 2 at $\mathrm{He}^{4}$ temperature is approximately $70 \mathrm{sec}$. The relatively long time constant greatly stabilizes the temperature on block 2 . The electrical connections to the sample are through four copper brackets which are thermally in contact but electrically insulated from the block. The leads connecting the four copper brackets to block 1 are niobium wires. The contribution to thermal conductance by these superconducting leads is negligible.

The sapphire substrate is glued onto block 2 with GE 7031 varnish to get a good thermal coupling. The vacuum can is sealed with indium 0-ring and evacuated. Hydrogen exchange gas is added into the can to reach a pressure about 7 Torr. After the can is immersed in liquid nitrogen, it takes about one hour for block 2 to reach thermal equilibrium. After the temperature reaches $77 \mathrm{~K}$, the liquid nitrogen is poured out of the dewar, and the liquid helium is transferred. The helium transfer in the beginning should be slow enough not to let the exchange gas condense immediately. The transfer time is usually half an hour. The SQUID is still hot after the helium is transferred. It takes one more 
hour for the SQUID to become supexconducting, and three more hours for block 2 to reach $4.2 \mathrm{~K}$. We pump down the helium bath to $3.2 \mathrm{~K}$. The temperature is regulated by a Rochlin bridge ${ }^{37}$ to within \pm 50 uk. The heater on block 2 raises the temperature to the superconducting transition of the tin film. The current supply for the heater was made up of two dc current sources in paralle1. These current sources are referenced to standard mercury cells. One has a ten-turn pot with continuously adjustable current range from 0 to $1 \mathrm{~mA}$. The other has an adjustable range from 0 to $10 \mu \mathrm{A}$. The temperature of block 2 is measured with a carbon resistance thermometer. The exact transition point of the sample is found as follows: when the film is superconducting, I is shorted by the sample and produces no SQUTD response. We maintain a constant $I_{1}$, say $2 \mu \mathrm{A}$, and keep $I_{2}=0$. Then we increase the heater current gradually. The SQUID output has a sudden change as the temperature of the tin film reaches the transition region where $I_{1}$ is divided between the two branches $R$ and $R_{S T D}$, resulting in a net current increase in the. SQUTD input coil $L_{1}$. In the feedback mode, the current change in $L_{i}$ is further cancelled by the feedback current. The fine adjustment knob in the heater current supply is used to bias the temperature of the tin film in the middle of the superconducting transition. The transition width of the tin film is typically $4 \mathrm{mK}$. The equilibrium temperature fluctuation in the tin film is of the order of $10 \mu \mathrm{K}$. This is why the temperature stabilization is so important in the noise measurement. With the long time constant in block 2, one has to be patient in adjusting the heater current. After reaching the steady state, the temperature of the tin film can stay there for at least 10 minutes without any 
by the interactions between the modulation coil, input coil, and the SQUID. A numerical calculation can be found in the work by Clarke and Tesche. 36

A schematic diagram of the SQUID insert is shown in Fig. 19. The SQUID system and the sample are mounted inside a vacuum can. The SQUID and the standard resistor are on copper block 1, which has a good thermal coupling to the $\mathrm{He}^{4}$ bath. The sample is on copper block 2 , which is supported by two thin wall stainless steel tubes to block 1 . A thin copper wire links the two blocks so that the measured time constant for block 2 at $\mathrm{He}^{4}$ temperature is approximately $70 \mathrm{sec}$. The relatively long time constant greatly stabilizes the temperature on block 2 . The electrical connections to the sample are through four copper brackets which are thermally in contact but electrically insulated from the block. The leads connecting the four copper brackets to block 1 are niobium wires. The contribution to thermal conductance by these superconducting leads is negligible.

The sapphire substrate is glued onto block 2 with GE 7031 varnish to get a good thermal coupling. The vacuum can is sealed with indium 0-ring and evacuated. Hydrogen exchange gas is added into the can to reach a pressure about 7 Tor $x$. After the can is immersed in liquid nitrogen, it takes about one hour for block 2 to reach thermal equilibrium. After the temperature reaches $77 \mathrm{~K}$, the liquid nitrogen is poured out of the dewar, and the liquid helium is transferred. The helium transfer in the beginning should be slow enough not to let the exchange gas condense immediately. The transfer time is usually half an hour. The SQUID is still hot after the helium is transferred. It takes one more 
hour for the SQUID to become superconducting, and three more hours for block 2 to reach $4.2 \mathrm{~K}$. We pump down the helium bath to $3.2 \mathrm{~K}$. The temperature is regulated by a Rochlin bridge ${ }^{37}$ to within $\pm 50 \mu \mathrm{K}$. The heater on block 2 raises the temperature to the superconducting transition of the tin film. The current supply for the heater was made up of two dc current sources in parallel. These current sources are referenced to standard mercury ce11s. One has a ren-turn pot with continuous ly adjustable current range from 0 to $1 \mathrm{~mA}$. The other has an adjustable range from 0 to $10 \mu \mathrm{A}$. The temperature of block 2 is measured with a carbon resistance thermometer. The exact transition point of the sample is found as follows: when the film is superconducting, $I_{1}$ is shorted by the sample and produces no SQUID response. We maintain a constant $I_{1}$, say $2 \mu \mathrm{A}$, and keep $I_{2}=0$. Then we increase the heater current gradually. The SQUID output has a sudden change as the temperature of the tin film reaches the transition region where $I_{1}$ is divided between the two branches $R$ and $R_{S T D}$, resulting in a net current increase in the. SQUID input coil $L_{1}$. In the feedback mode, the current change in $L_{i}$ is further cancelled by the feedback current. The fine adjustment knob in the heater current supply is used to bias the temperature of the tin film in the middle of the superconducting transition. The transition width of the tin film is typically $\&$ m. The equilibrium temperature fluctuation in the tin film is of the order of $10 \mu \mathrm{k}$. This is why the temperature stabilization is so important in the noise measurement. With the long time constant in block 2, one has to be patient in adjusting the heater current. After reaching the steady state, the temperature of the tin film can stay there for at least 10 minutes without any 
noticeable drift at the SQUID output.

\section{MEASUREMENT OF NOISE POWER SPECTRUM}

The noise power spectrum of the tin film at the superconducting transition was measured with a PDP-11 computer. The output signal from the SQUID system was connected to the input of the computer. The computer was programmed to sample the data and Fourier transform them. The noise power spectrum was obtained by taking the square of the Fouriertransformed data and dividing them by the sampling time. The ensemble average of many runs reduced the standard deviation and obtained a smooth spectrum. It can be shown that the noise power spectrum obtained in this way is the same as measuring the autocorrelation function directly and taking the cosine transform. Of course, a low-pass filter had to be used before taking FFT to avoid aliasing.

The measured noise power spectrum is shown in Fig。 20. The solid line is the calculated Johnson noise level of the standard resistor and the sample. Apparently, the lower spectrun with $I_{1}=0$ is dominated by the Johnson noise. The upper spectrum is the current dependent noise, which is white in the frequency range $1 \mathrm{~Hz}$ to $300 \mathrm{~Hz}$. The rolloff beyond $300 \mathrm{~Hz}$ is not intrinsic but due to the electronics as can be seen from the Johnson noise spectrum. The magnitude of the white noise spectrum is: $\mathrm{S}_{\mathrm{v}}(\xi) / \mathrm{V}^{2}=1.3 \times 10^{-9} \mathrm{~Hz}^{-1}$. The corresponding temperature fluctuation $\mathrm{S}_{\mathrm{T}}(f)=9.4 \times 10^{-15} \mathrm{~K}^{2} \mathrm{~Hz}-1$. From the measured noise power spectrum, we know that the tin film should be strongly coupled to the substrate. ${ }^{3}$ A quantitarive prediction of the spectrum from the remperature fluctuation model is not avallable because of the 
uncertainty introduced by the boundary impedance between the fllm and the substrate.

The resistance of the film was measured using $\mathrm{Eq} \cdot(8.1)$ for different bias current and different block temperatures. The result is plotted in Fig. 21. When the bias current is increased, the $R$ vs. Tblock curve tends to shift to the left and become sharper. This is due to the combination of self-heating and the magnetic field generated by the bias current. If we neglected the magnetic field effect, the thermal conductance calculated from $R$ vs. $I_{1}$ for a fixed block temperature would be underestimated by considering the self-heating alone. At $T_{\text {block }}=3.7204 \mathrm{~K}$, the thermal conductance (neglecting the magnetic field effect) varies from $1.8 \times 10^{-8} \mathrm{WK}^{-1}$ to $5.0 \times 10^{-7} \mathrm{WK}^{-1}$ as the bias current is increased from $1 \mu \mathrm{A}$ to $10 \mu \mathrm{A}$. If we assume a constant thermal conductance, the shift of $\mathrm{R}$ VS. $\mathrm{T}_{\mathrm{block}}$ curve due to the magnetic field produced by the bias current is less than linear as the bias current is increased.

The data given in Fig. 21 have been replotted in Fig. 22 with $\mathrm{T}_{\text {block }}$ as the parameter. The nonlinearity in the $I$ vs. $V$ curve is obvious. We attribute it to the self-heating and the decrease of transition temperature with increasing bias current (magnetic field effect). However, it is very difficult to find out the fractional contribution for each of these two effects.

According to the temperature fluctuation model, the voltage noise power spectrum is proportional to $\mathrm{I}^{2}$ if $\mathrm{dR} / \mathrm{dT}$ is a constant. The measured voltage noise power spectrum for a fixed block temperature rises faster than $I^{2}$ as shown in Fig. 23. This is due to the 
self-heating effect which increases $d R / d T$.

\section{MEASUREMENT OF AUTOCORRELATION FUNCTION FROM MACROSCOPIC DECAY}

The response of the film to a step-function thermal perturbation was measured using the circuit shown in Fig. 24. The bias current of the film was controlled by the switch. The time constant in flipping the switch is about $100 \mathrm{~ns}$, which is due to the discharge of the lead shunt capacitance through the sample. The power dissipation in the film is $I_{1}^{2} R$. After the flipping of the switch, the thermal power generated in the film by the bias current was changed. The resistance of the film would relaxate to the new value gradually. This resistance change as a function of time can be read out through the SQUID. The slewing rate of the SQUID is approximately $10^{4} \phi_{0} / \mathrm{sec}$. If the current change is less than $10 \mu \mathrm{A}$, the SQUID can follow the whole decay curve without any flux jumping.

The output signal from the SQUID was fed to the computer through PAR TM 113 amplifier and Krohn-Hite 3320 filter. The PAR TM 113 was dc coupled with a high Erequency roll-off of $3 \mathrm{k} \mathrm{Hz}$. The de off-set, polarity, and the gain were adjusted so that the output voltage before Elipping the switch was between $0.5 \mathrm{~V}$ and $1 \mathrm{~V}$, and the steady state voltage after flipping the switch was around $6 \mathrm{~V}$. The Krohn-Hite 3320 had a gain of unity and a low pass Eour pole roll-off fxequency equal ro one half of the sampling frequency to avoid allasing in FFT。

The input signal was digitized with a voltage-to-frequency converter and frequency counter. The computer was programmed such that the intercuption signal (with Erequency equal to the sampling frequency) 
would stop the counter, read the number of counts, and reset the counter. There were 1024 channels in the program. The number of counts would not fill the first channel until the input voltage was greater than $1 \mathrm{~V}$. At the initial steady state, the input voltage was less than $1 \mathrm{~V}$. The computer would be in the idle mode. After the switch was flipped, the input voltage would rise to $6 \mathrm{~V}$. The information above $1 \mathrm{~V}$ was recorded into the 1024 channels. With a sampling frequency of $1 \mathrm{kHz}$, it took about $1 \mathrm{sec}$ to record the data. The recorded curve was then inverted and rescaled such that the first channel was equal to $\mathrm{kT}^{2} / \mathrm{C}_{\mathrm{y}}$ and the last channel was zero. The whole autocorrelation function was stored in the 1024 channels in the computer. We recorded the normalized decay curve several times and took the ensemble average to increase the signal-to-noise ratio. The error in the normalization of the response curve introduced by dropping the voltage below $1 \mathrm{~V}$ was less than $10 \%$. The FFT program then took the Fourier transform of these sampled data points. The real part corresponded to the cosine transform while the imaginary part corresponded to the sine transform.

The $\delta$-function response could be measured by applying a short duration heat pulse to the film and observing the decay of the resistance. But, unfortunately, the initial rise of the resistance was so fast that the SQUID electronics could not follow it. An alternative way to measure it was to take the time derivative of the step-function response curve. Since a $\delta$-function is the derlvative of a step-function, the response curve should hold the same relation. Mathematically it can be shown that the cosine transform of the derivative of a decay curve is related to the sine transform of the decay curve itself. 
Assuming $C(T)$ is the steperunction reponse, we get

$$
\int_{0}^{\infty} C^{\gamma}(\tau) \cos w T d \tau=-C(0)+\omega \int_{0}^{\infty} C(\tau) \sin \alpha \tau d \tau \text {. }
$$

A complete Fourier-transform of $C(\tau)$, which is the step-function response, gives information on the noise power spectra obtalned from both stepfunction and $\delta$ function pertuxbations.

In the FFT program, 1024 real points and 1024 imaginary points will generate 512 real and 512 Imaginary points in the absence of aliasing. When using FFT, we let the 1024 channels digitized from $C(\tau)$ be the real part. The imaginary part of input is zero. After Frt, we get 512 points from the cosine transform (real part) and 512 poincs from the sine transform (imaginaxy paxt). The resolution of the cosine transform can be improved by sacrificing the information in sine transform. If we assume $C(\tau)$ to be a symmertic function, that is, $C(-\tau)$ a $C(\tau)$, we can get 2048 digitized channels for $C(\mathrm{~T})$ by mapping it to the negative time axis, though we measured only 1024 channels. The cosine transform of these 2048 channels will give 102 h new chanels inctead of 512. But the sine transform gives sero because $C(\tau)$ is even while sin(wi) is odd. The resolution of the cosine transform has been increased by a factor of 2. But the information on the sine transform has been lost completely. We adopted the formex method since it produces the stepIunction and $\delta$-function responses simultaneously.

The decay program was checked with an RC discharge circuit. We know that the decay of $R C$ discharge is an exponential function of time. The Fourier transform can be expressed explicitly, 


$$
\int_{0}^{\infty} e^{-\tau / \tau_{0}} \cos w \tau d \tau=\frac{\tau_{0}}{1+\omega^{2} \tau_{0}^{2}}
$$

and

$$
\int_{0}^{\infty} e^{-\tau / \tau} 0 \sin w \tau d \tau=\frac{w \tau_{0}^{2}}{1+w^{2} \tau_{0}^{2}},
$$

If one chooses a proper sampling frequency, the measured points are in excellent agreement with the theoretical curve (see Fig. 25)。

We measured the response of the tin film to a step-functon perturbation. The decay curve as a function of time is plotted in Fig. 26. The cosine transforms of the step-function response and the $\delta$-function response (obtained from sine transform of step-function response) are plotted in Fig. 27. These two spectra can be approximately expressed by:

$$
\mathrm{S}_{\text {step }}(\mathrm{f})=\frac{3.5 \times 10^{-11}}{\mathrm{f}} \mathrm{K}^{2} \mathrm{~Hz}^{-1}
$$

and

$$
\mathrm{S}_{\delta}(f)=4 \times 10^{-13} \mathrm{k}^{2} \mathrm{~Hz}^{-1}
$$

The directly measured noise power spectrum is:

$S_{\mathrm{T}}(\mathrm{f})=9.4 \times 10^{-15} \mathrm{~K}^{2} \mathrm{~Hz}^{-1}$. It is obvious that the shape of the directly measured noise power spectrum is closer to the spectrum obtained from $\delta$-function perturbation. The magnitude is off by a factor of 40 . But this is due to the defect in finite channel FFT program. We will give a more detailed discussion on this topic in Sec. B of Chapter IX. 


\section{DISCUSSION OF THE NOISE EXPERIMENT}

\section{A. THERMAL COUPLING BETWEEN FILM AND SUBSTRATE}

There are two mechanisms which account for the thermal conductance between a thin metal film and a dielectric substrate: (i) A direct excitation of phonons in the substrate by electrons in the film. (ii) The thermal phonon transmission through the metal-insulator interface.

Cheeke ${ }^{38}$ has found that the electronic contribution is not important compared with the transmission due to the acoustic mismatch of thermal phonons. The transmission of phonons across a solid-solid interface has been investigated by several people。 $39,40,41$ The experiments show that. the acoustic-mismatch model is reasonably accurate. The assumptions made in this model include: the metal film is isotropic; the thermal phonons in the thin film are in thermal equilibrium at a certain temperature $\mathrm{T}_{1}$; the density of states of the phonons can be described by Debye model with the same cut-off wavevector $\left|q_{\max }\right|$ for both transverse and longitudinal phonons; the continuity of particle displacement and stress is assumed at the interface, giving the relation between the transmitted wave and the incident wave analogous to Snell's Law in optics.

By similar calculations leading to the Stefan-Boltzman law for photons, an equivalent blackbody radiation formula for phonons can be derived. The thermal radiation power transferred pex unit area from a film at temperature $T_{1}$ to a substrate at cemperature $T_{0}$ is ${ }^{40}$

$$
\frac{P\left(T_{1}, T_{0}\right)}{A}=\frac{\pi^{2}}{120 \hbar^{3}}\left\{\frac{e_{l}^{(10)}}{C_{l}^{(1) 2}}+\frac{e_{t_{1}}^{(10)}+e_{t_{2}}^{(10)}}{C_{t}^{(1) 2}}\right\} k_{B}^{\left(T_{1}\right.}\left(T_{1}^{4}-T_{0}^{4}\right)
$$


where $c$ is the phonon phase velocity; $e$ is the emisstvity of phonons from the film into the substrate due to acoustic-mismatch; 1 and $t$ refer to longitudinal and transverse phonons. The emissivity e can be computed numerically from the boundary conditions introduced at the interface. The sound velocity $c$ has already been well known.

For a tin film on a saphire substrate, the calculated values for emissivities $\operatorname{are}^{40} e^{(10)}=0.0809$ and $e_{t}(10)=e^{(10)} t_{t 2}=0.0702$. The sound velocities in tin are $c_{\ell}(1)=3.32 \times 10^{3} \mathrm{~m} / \mathrm{sec}$ and $c_{\mathrm{t}}^{(1)}=$ $1.67 \times 10^{3} \mathrm{~m} / \mathrm{sec}$. The thermal conductance per unit $\mathrm{cm}^{2}$ can be calculated for a sma11 temperature difference $\left(\mathrm{T}_{1}-\mathrm{T}_{0} \ll \mathrm{T}_{1}, \mathrm{~T}_{0}\right)$ at the superconducting transition temperature of tin. By substituting the numerical values into $\mathrm{Eq} .(9.1)$, we get

$$
\frac{P\left(T_{1} T_{0}\right)}{A}=3.16\left(T_{1}-T_{0}\right)
$$

The thermal conductance due to acoustic mismatch is

$$
G_{\text {acoustic mismatch }}=\frac{P\left(T_{1}, T_{0}\right)}{\left(T_{1}-T_{0}\right)}=3.16 \mathrm{~A} \mathrm{WR}^{-1} \text {. }
$$

For a film with dimensions $500 \mu \times 12 \mu \times 1000 \AA$, the thermal conductance from acoustic mismatch is $G_{\text {acoustic mismatch }}=1.9 \times 10^{-4} \mathrm{WK}^{-1}$. The actual thermal conductance could be less because the coupling at the interface may not be idea1. However, the calculated thermal conductance is an upper limit. No thermal coupling can be better than the result derived from the acoustic mismatch model. Now we are going to consider the other extreme case, the chermal conductance of a freely suspended film. Assume we measure the resistance 
of the film by the four-terminal method. When the bias current is increased, the resistance will increase more rapidly than linearly due to the self-heating. From the R vs. T curve, we can find the temperature change of the film. The ratio of the power dissipated in the film to the temperature change is the thermal conductance. This thermal conductance can be calculated from the diffusion equation. Since the temperature inside the film is not uniform, that is, the resistivity is a function of position, the power dissipated inside the film is

$$
P(x)=\alpha_{1}\left(T(x)-T_{0}\right)+\alpha_{2}
$$

where

$$
\alpha_{1}=\frac{I^{2}}{(w t)^{2}} \frac{d \rho}{d t} .
$$

and

$$
\alpha_{2}=\frac{I^{2} \rho_{0}}{(w t)^{2}}
$$

Here $T_{0}$ is the temperature at both ends of the film: $w$, $t$ are the width and thickness of the film; $\rho, \rho_{0}$ are the resistivities of the film at temperature $\mathrm{T}_{8} \mathrm{~T}_{0}$; I is the dc bias current, We have assumed a temperature gradient only along the longest dimension of the film. In the steady state, the thermal diffusion equation becomes

$$
-C_{v} D \frac{d^{2} T}{d x^{2}}=p=\alpha_{1}\left(T-T_{0}\right)+\alpha_{2},
$$

where $C_{v}$ is the specific heat, and $D$ is the diffusion constant. By solving Eq. (9.5) and matching the boundary conditions $\mathrm{T}(-1 / 2 \ell)=$ $\mathrm{T}(1 / 2 \ell)=\mathrm{T}_{0}$, we get

$$
T_{(x)}=\frac{\alpha_{2} \cos (\beta x)}{\alpha_{1} \cos (\beta l / 2)}-\frac{\alpha_{2}}{\alpha_{1}}+T_{0} .
$$


where

$$
\beta=\sqrt{\frac{\alpha_{1}}{C_{v} D}}
$$

the average temperature of the film is

$$
\bar{T}=\frac{1}{l} \int_{-\frac{l}{2}}^{\frac{l}{2}} T(x) d x=\frac{2 \alpha_{2}}{l \beta \alpha_{1}} \tan \frac{\beta \ell}{2}-\frac{\alpha_{2}}{\alpha_{1}}+T_{0} .
$$

The total power dissipation is

$$
\mathrm{p}_{\text {tot }}=w t \int_{-\frac{\ell}{2}}^{\frac{\ell}{2}} \mathrm{P}(\mathrm{x}) \mathrm{dx}=\operatorname{lwt}\left(\alpha_{1} \overline{\mathrm{T}}-\alpha_{1} \mathrm{~T}_{0}+\alpha_{2}\right) \text {. }
$$

From Eq. (9.7) and Eq. $(9.8)$, we can eliminate $\alpha_{2}$ to obtain

$$
P_{\text {tot }}=\frac{\frac{2 \alpha_{1} w t}{\beta} \tan \frac{\beta l}{2}}{\frac{2}{\beta l} \tan \frac{\beta l}{2}-1}\left(\bar{T}-T_{0}\right)=G_{\text {free }}\left(\bar{T}-T_{0}\right) \text {, }
$$

and

$$
G_{\text {free }}=\frac{\frac{2 \alpha_{1} w t}{\beta} \tan \frac{\beta l}{2}}{\frac{2}{\beta l} \tan \frac{\beta l}{2}-1} .
$$

When $B l / 2$ is sma11, we can expand tan $\beta l / 2$ using the formula

$\tan x \approx x+x^{3} / 3$. The error is less than $10 \%$ as long as $x<0.9$. We find

$$
G_{\text {free }} \approx \frac{12 C_{v} D w t}{l}=\frac{12 \mathrm{~K} w t}{l}
$$

where $K$ is the thermal conductivity。 The Wiedemann-Franz law states that for metals at not too low temperatures the ratio of thermal conductivity to electrical conductivity is directly proportional to the temperature. The constant of proportionality is called the Lorenz number, and is independent of the metal studied:

$$
\frac{K}{\sigma T}=\frac{\pi^{2}}{3}\left(\frac{k_{B}}{e}\right)^{2}=L=2.45 \times 10^{-8} \mathrm{Wr} / \mathrm{K}^{2} .
$$

At low temperature $(T \ll \theta)$, the value of $L$ tends to decrease. This 
is due to the different relaxation times involved in $K$ and $\sigma$. The Lorenz number $L$ should be modified as follows:

$$
L=\frac{\pi^{2}}{3}\left(\frac{k_{B}}{e}\right)^{2} \frac{\tau_{t h}}{\tau_{e l}}
$$

The ratio ${ }^{\tau} \mathrm{th} / \tau_{\mathrm{e}}$ decreases at low temperature. For pure copper ${ }^{42}$ near $15 \mathrm{~K}$, the value $\tau_{t h} / T_{\text {el }}$ is about 0.1 .

By combining $\mathrm{Eq} \cdot(9.11)$, Eq. $(9.12)$, and $\mathrm{Eq} \cdot(9.13)$, we get:

$$
G_{\text {Eree }} \approx \frac{12 w t}{l} \frac{\pi^{2}}{3}\left(\frac{k_{B}}{e}\right)^{2} \text { or } \frac{\tau_{t h}}{\tau_{e 1}} \text {. }
$$

For tin film with dimensions $500 \mu \times 12 \mu \times 1000 \AA$, the resistance just above the superconducting transition tempexature is $2.55 \AA$. The electrical conductivity is $1.63 \times 10^{6} \mathrm{rom}^{-1} \mathrm{~cm}^{-1}$. At $3.7 \mathrm{~K}, \mathrm{Eq} \cdot(9.14)$ becomes

$$
G_{\text {free }}=4.3 \times 10^{-7^{\tau} \mathrm{th}} \frac{\mathrm{WK}^{-1}}{\tau_{\mathrm{e}}} .
$$

The measured thermal conductance as discussed in Chapter VIII sec. $C$ varies from $1.8 \times 10^{-8}$ WK $^{-1}$ to $5.0 \times 10^{-7} \mathrm{WK}^{-1}$ as the bias current increases from $1 \mathrm{HA}$ to $10 \mathrm{HA}$. These measurements have neglected the shift of the transition temperature by the magnetic field. The actual thermal conductance should be considerably bigger than $5.0 \times 10^{-7} \mathrm{WK}^{-1}$. The calculated thermal conductance along the film is $4.3 \times 10^{-7} \mathrm{WK}^{-1}$ in the normal state with $\tau_{t h} / \tau_{e 1}=1$. In the middle of the transition, part of these electrons will be condensed to Cooper pairs and $\tau^{t} h^{/ \tau}$ el should be less than 1 . The thermal conductance along the f1lm is considerably less than $4.3 \times 10^{-7} \mathrm{WK}^{-1}$. Therefore, the total thermal 
conductance of the film is more than the heat flow along the film can account for. The extra thermal conductance is from the coupling between the film and the substrate. In our sample the thermal conductance is between $5.0 \times 10^{-7} \mathrm{WK}^{-1}$ and $1.9 \times 10^{-4} \mathrm{WK}^{-1}$. Most of the heat generated inside the sample is conducted into the substrate rather than along the film. These samples have good thermal coupling to the substrate. According to Clarke and Hsiang's experiment, ${ }^{3}$ a film which is strongly coupled to the substrate shows a white noise power spectrum at low Exequency. This is consistent with our measurement.

\section{B. NORMALIZATION OF THE DECAY CURVE}

The Fourier transform of a function $C(\tau)$ can be calculated by sampling the function and then using FFT program. The sampled data $\mathrm{C}_{\mathrm{S}}(\tau)$ is related to the continuous function $\mathrm{C}(\tau)$ through the shah function $\operatorname{III}(x): 43$

$$
C_{S}(\tau) \equiv \operatorname{III}(\tau / \Delta \tau) C(\tau)
$$

where

$$
\operatorname{III}(x) \equiv \sum_{n=\infty}^{\infty} \delta(x-n) .
$$

Here $\Delta \tau$ is the sampling interval. The function $C_{S}(\tau)$ is zero except at these points when $\tau=0, \pm \Delta \tau, \pm 2 \Delta \tau, \ldots$. The Fourier transform of a shah function is still a shah function

$$
\operatorname{F}\{\operatorname{II}(a x)\}=(1 /|a|) \operatorname{III}(\sigma / a)
$$

Assume $S_{S}(f)$ and $S(f)$ are the Fourier transforms of $C_{S}(\tau)$ and $C(\tau)$, respectivelye Since $C_{S}(\tau)$ is the product of $C(\tau)$ and III $(\tau / \Delta \tau)$, 
$S_{S}(f)$ should be the convolution of $S(f)$ and $F\{I I I(\tau / \Delta \tau)\}$ 。

$$
\begin{aligned}
S_{S}(f) & =F\{I I I(\tau / \Delta \tau)\} \& C(\tau) \\
& =\sum_{n=-\infty}^{\infty} S[f-n(\Delta f)],
\end{aligned}
$$

where $\Delta f=1 / \Delta \tau$. If $\Delta f \geq 2 f_{\text {max }}$ (no aliasing), $S_{S}(f)$ represents $S(f)$ everytime $f$ equals $n(\Delta f)$ for all integers $n$. Theoretically, the data sampling will not cause any errors for an infinitely long sampling series. But in the real case, there are only a finite number of channels to record the data.

The FFT program pexforms Fourier transform on $N$ channels. The mathematical formula is

$$
\begin{aligned}
H(k) & =\sum_{n=0}^{N-1}\left[h_{\Upsilon}(n)+j h_{i}(n)\right] e^{-j 2 \pi n k / N,}, \\
\text { or } \quad H_{x}(k) & =\sum_{n=0}^{N-1}\left[h_{\Upsilon}(n) \cos (2 \pi n k / N)+h_{i}(n) \sin (2 \pi n k / N)\right], \\
\text { and } \quad H_{i}(k) & =\sum_{n=0}^{N-1}\left[h_{i}(n) \cos (2 \pi n k / N)-h_{\Upsilon}(n) \sin (2 \pi n k / N)\right],
\end{aligned}
$$

where the subscripts $r$ and $i$ refer to the real and imaginary part of the function. When $h_{1}(n)=0, H_{x}(k)$ corresponds to the cosine transform of $h_{Y}(n)$ and $H_{i}(k)$ corresponds to the sine transform of $h_{r}(n)$. If $h_{r}$ represents the autocorrelation function, $H_{r}$ is the noise power spectrum. The total noise power in the Erequency band covered by FET is the integral of $H_{r}$ over the frequency band, or, in digitized form,

$$
\sum_{k=0}^{N=1} H_{r}(k)=\sum_{k=0}^{N-1} \sum_{n=0}^{N-1} h_{Y}(n) \cos (2 \pi n k / N)=N h_{Y}(0)
$$


which is the first channel in the autocorrelation function.

The finite channel FFT program can only give the spectrum information in a limited frequency range. In the equilibrium temperature fluctuation model we can get accurate noise power spectrum by normalizing the first channel of the autocorrelation function to $\mathrm{kT}^{2} / \mathrm{C}_{\mathrm{v}}$ and then using FFT program provided most of the noise falls in the frequency range of FrT. For a 1024 channel FFT with sampling frequency $f_{s}$, it covers a frequency range about three decades, from $f_{\mathrm{S}} / 1024$ to $\xi_{\mathrm{S}} / 2$. If we assume an $1 / f$ noise power spectrum is observed, the total noise Within this frequency band is

$$
\int_{f_{S} / 1024}^{f_{S} / 2} \frac{a}{f} d f=a \ln 512
$$

We know the total noise cannot diverge in the equilibxium temperature fluctuation model. The $1 / f$ spectrum must roll off at both high and low frequencies. Even if the $1 /$ f region extends another three decades, the normalization constant is only off by a factor of two. The exror introduced by the FFT program is not very serious.

For a Lorentzian or Lorentzian-1ike spectrum, that is, one which is flat at low frequencies and then rolled of fastex than $1 / f$ above some knee frequency $f_{0}$, the $\mathbb{N}$ channel FFT with sampling frequency $f_{s}$ can regenerate the exact spectrum from the autocorrelation function only when the spectrum between $\xi_{S} / N$ and $f_{S} / 2$ contributes most of the noise, otherwise the magnitude of the spectrum will be inaccurate. For example, if we choose $f_{S} \gg 2 \hat{f}_{0}$ and $f_{S} / N \ll f_{0}$ in a Lorentzian spectrum, the spectrum well above $f_{0}$ and well below $f_{S} / \mathbb{N}$ will be negligible compared with the total noise due to the fast roll-off $\left(\sim 1 / f^{2}\right)$ at high frequencies 
and narrow bandwidth $\left(\sim f_{S} / N\right)$ at low frequencies. Assume the total noise is normalized to 1:

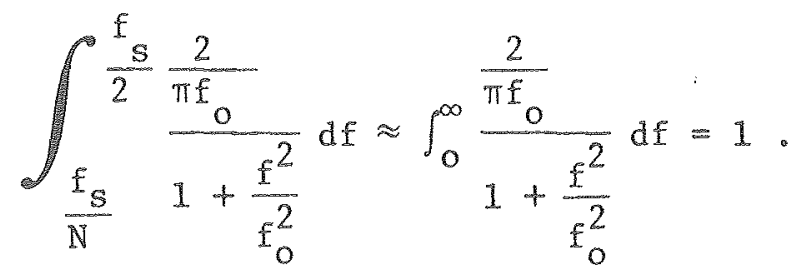

At low frequency $\left(f \ll f_{0}\right)$, the spectrum is white and given by $2 / \pi f_{0}$. But if we choose $f_{S}=f_{0} / 1000$ and $N=1024$, the FFT program will give a white spectrum of magnitude $2000 / f_{0}$ between $f_{0} / 1024000$ and $f_{0} / 2000$. The shape of the spectrum is still the same, but the magnitude is bigger by a factor of $1000 \pi$.

In the decay experiment discussed in Chapter VIII, the noise power spectrum obtained from $\delta$-function perturbation is white in the frequency range 1 to $500 \mathrm{~Hz}$. The magnitude of the spectrum cannot be correct if the rol1-off happens at a frequency considerably higher than $500 \mathrm{~Hz}$. According to the equilibrium temperature fluctuation mode1, the overall temperature fluctuation should be $(\Delta T)_{\text {tot }}^{2}=\mathrm{kT}^{2} / \mathrm{C}_{\mathrm{v}}=2.2 \times 10^{-10^{2}}$. The directly measured temperature fluctuation spectrum from Chapter VIII Sec. $C$ is $(\Delta T)_{\mathrm{S}}^{2} \equiv 9.4 \times 10^{-15} \mathrm{~K}^{2} \mathrm{~Hz}^{-1}$. An effective bandwidth of $23 \mathrm{kHz}$ is anticipated. In the decay experiment, the sampling frequency is $1 \mathrm{kHz}$. The FFT gives a maximum frequency of $500 \mathrm{~Hz}$. The spectrum below $500 \mathrm{~Hz}$ constitutes only $2.2 \%$ of the total noise. It is not surprising that the spectrum obtained from decay experiment is overestimated by a factor of 40 。

We certainly will be able to improve the decay experiment by sampling at much higher frequency $(>23 \mathrm{kHz})$. But, unfortunately, the program does not function with a sampling frequency above $2 \mathrm{KHz}$. The reason is 
that the sampling period in this program has to be long enough such that the computer returns to the waiting position before the next interrupting signal arrives. Even if we use the maximum sampling frequency (2 $\mathrm{KHz}$ ) possible for this program, the spectrum we get may still be off by a factor of 20. In addition, the present SQUTD system has a roll-off frequency at $500 \mathrm{~Hz}$. A transformer should be used instead of the tank circuit in SQUID electronics to increase the bandwidth of the read-out system.

\section{CONCLUSIONS}

The decay experiment for the tin film on sapphire substrate shows that the cosine transform of the $\delta$-function decay corresponds to the noise power spectrum. The noise driving term in this system is a random heat current flowing around inside the sample, and the uncorrelated model is appropriate to describe the fluctuations. This phenomenon is different from that observed at room temperature, in which case the cosine transform of the step-function decay gives the correct noise power spectrum, and the correlated model is applicable. For a freely suspended tin fiber at the superconducting transition, the uncorrelated model is again the correct one.

At low temperature, the substrate plays an important role in determining the shape of the noise power spectrum, but does not affect the nature of the random noise driving term. The existing experiments strongly indicate that the noise driving term in the metal thin film is a function of temperature xather than the boundary conditions introduced to the film. The correlated model applies at room temperature, while the uncorrelated model applies at liquid helium temperature. 
ACKNOWLEDGMENT

I would 1ike to thank my thesis advisor, Professor John Clarke, for his contant guidance, advice, and patience throughout the course of this work. I also would like to thank Professor Paul L. Richards for many helpful discussions on far-infrared physics and techniques, and for the use of his experimental equipment. My other colleagues in Clarke's group and Richards' group are gratefully acknowledged for innumerable useful discussions and the creation of a stimulating atmosphere.

The technical assistance received from the professional shops in the Physics Department, the Lawrence Berkeley Laboratory, and the Space Sciences Laboratory has been of great value. I am also grateful to Gloria C. Pelatowsky and Michele Marram for their careful preparation of this manuscript.

Finally, I wish to thank my wife, Scarlett, for her continued understanding and encouragement.

This work was performed under the auspices of the U.S. Department of Energy。 
APPENDIX

Conslder a bolometer with heat capacity $\mathrm{C}$ at temperature $\mathrm{T}$ linked to a copper mount at temperature $T$ via a thermal conductance G. The copper mount with heat capacity $C_{m}$ is linked to He bath at temperature $T_{H e}$ with a thermal conductance $G_{m}$. The signal from the bolometer is fed back to the heater on the coppex mount to stabilize the temperature. The loop gain in the feedback circuit is $\theta_{\text {. For }}$ external perturbation with thermal power $\Delta \mathrm{P}_{B} \cos \left(w t+\delta_{1}\right)$ incident onto the bolometer and helium bath temperature fluctuation $\Delta \mathrm{T}_{\mathrm{He}} \cos \left(\mathrm{wt}+\delta_{2}\right)$, the equations for conservation of energy become:

$$
C \frac{d T}{d t}=-G\left(T-T_{m}\right)+\varepsilon \Delta P_{B} \cos \left(w t+\delta_{l}\right)
$$

$C_{m} \frac{d T}{d t}=-G_{m}\left(T_{m}-T_{H e}-\Delta T_{H e} \cos \left(w t+\delta_{2}\right)\right)-G_{m} \alpha T+G\left(T-T_{m}\right)$

By eliminating $\mathrm{T}_{\mathrm{m}}$ in $\mathrm{Eq} \cdot\left(\mathrm{A}_{\cdot} 1\right)$ and $\mathrm{Eq} \cdot\left(\mathrm{A}_{.} 2\right)$, we get:

$$
\begin{aligned}
& \frac{\mathrm{d}^{2} \mathrm{~T}}{\mathrm{~d} t^{2}}+\left(\frac{1}{\tau}+\frac{1}{\tau_{\mathrm{m}}}+\frac{\mathrm{G}}{\mathrm{C}_{\mathrm{m}}}\right) \frac{\mathrm{dT}}{\mathrm{dt}}+\frac{\alpha+1}{\tau \tau_{\mathrm{m}}} \mathrm{T}=-\frac{\varepsilon \Delta \mathrm{P}_{\mathrm{B}}}{\mathrm{C}} \mathrm{w} \sin \left(\mathrm{wt}+\delta_{1}\right)+ \\
& \left(\frac{1}{\tau_{\mathrm{m}}}+\frac{\mathrm{G}}{\mathrm{C}_{\mathrm{m}}}\right) \frac{\Delta \mathrm{P}_{\mathrm{B}}}{\mathrm{C}} \cos \left(w t+\delta_{1}\right)+\frac{1}{\tau \tau_{\mathrm{m}}} \Delta \mathrm{T}_{\mathrm{He}} \cos \left(w t+\delta_{2}\right)+\frac{1+\alpha}{\tau \tau_{\mathrm{m}}} \mathrm{T}_{\mathrm{He}},
\end{aligned}
$$

where $\tau=C / G$ and $\tau_{m}=C_{m} / G_{m}$. When $C_{m} \gg C, G_{m} \gg G, \tau_{m} \gg \tau$ and $a \gg 1, \mathrm{Eq} \cdot(\mathrm{A} .3)$ can be simplified:

$$
\begin{aligned}
& \frac{\mathrm{d}^{2} \mathrm{~T}}{\mathrm{~d} t^{2}}+\frac{1}{\tau} \frac{\mathrm{dT}}{\mathrm{d} t}+\frac{\alpha}{\tau \tau_{\mathrm{m}}} \mathrm{T}=\frac{\varepsilon \Delta \mathrm{P}_{\mathrm{B}}}{\mathrm{C}} \sqrt{\mathrm{w}^{2}+\frac{1}{\tau_{\mathrm{m}}^{2}}} \cos \left(\mathrm{wt}+\delta_{1}\right) \\
& +\frac{\alpha}{\tau \tau_{\mathrm{m}}} \mathrm{T}_{\mathrm{He}}+\frac{1}{\tau \tau_{\mathrm{m}}}\left(\Delta \mathrm{T}_{\mathrm{He}} \cos \left(\mathrm{wt}+\delta_{2}\right)\right),
\end{aligned}
$$


and

$$
\delta_{1}^{\prime}=\tan ^{-1} \frac{w}{\frac{1}{\tau_{m}}+\frac{G}{C_{m}}}
$$

The solution for $\mathrm{T}$ is

$$
T=\frac{\left(\varepsilon \Delta \mathrm{P}_{\mathrm{B}} / G\right)\left(1+w^{2} \tau_{\mathrm{m}}^{2}\right)^{1 / 2} \cos \left(\omega t+\delta_{1}^{\prime \prime}\right)+\Delta \mathrm{T}_{\mathrm{He}} \cos \left(\omega t+\delta_{2}^{\prime \prime}\right)}{\tau \tau_{\mathrm{m}}\left[\left(w^{2}-w_{0}^{2}\right)^{2}+w^{2} / \tau^{2}\right]^{1 / 2}}+T_{\mathrm{He}}
$$

where $\quad w_{0}^{2}=\frac{\alpha}{\tau \tau_{m}}$

$$
\begin{aligned}
& \delta_{1}^{\prime \prime}=\delta_{1}^{\prime}+\theta \\
& \delta_{2}^{\prime \prime}=\delta_{2}+\theta \\
& \theta=\tan ^{-1} \frac{w}{\left(w^{2}-w_{0}^{2}\right) \tau}
\end{aligned}
$$




\section{REFERENCES}

1. C. Kittel, "Thermal Physics," pp. 245-262, John Wiley and Sons, Inc, New York, (1969).

2. E.H. Putley, J. Sci. Instr. 43, 857 (1966).

3. J。 Clarke and T. -Y. Hsiang, Phys. Rev, B13, 4790 (1976).

4. D.H. Andrews, W.F. Brucksch, JR, W.T. Ziegler, and E.R. Blanchard, Rev. Sci. Instrum. 13, 281 (1942); D.H. Martin and D. Bloor, Cryogenics 1, 159 (1961); D. Bloor, T.J. Dean, G. O. Jones, D.H. Martin, P.A. Mawer, and C.H. Perry, Proc, Roy, Soc, A260, 510 (1961); C.L. Bextin and K. Rose, J.Appl. Phys. 39, 2561 (1968); M.K. Maul, M.W.P. Standberg, and R.L. Kyhl, Phys. Rev. 182, 522 (1969); M.K. Maul and M.W.P. Standberg, J. Appl. Phys. 40, 2822 (1969): I.A. Khrebtov, NoM。 Gropshtein, and GoA. Zaitsev, Prib. i Tek.Eksp. 1, 247 (1971); C.L. Bertin and K. Rose, J. Appl. Phys. 42, 163 (1971); R.M. Katz and K. Rose, Proc。IEEE 61, 55 (1973); N.A. Pankratov, G.A. Zaitsev, and I.A. Khrebtov, Cryogenics 13, 497 (1973); G. Gallinaro and R. Varone, Cryogenics 15, 292 (1975)。

5. Jo Clarke, G.I. Hoffer, B.L. Richards, and $N_{0}-H_{0}$ Yeh, Low temperature physics - LT 14, edited by M. Krusius and M. Vuorio (Elsevier, New York, 1975), Vol. 4, pp. 226-229; J. Clarke, P.L. Richards, and N.-H. Yeh, Appl. Phys, Letr. 30, 664 (1977).

6. J. Clarke, G.I. Hoffer, R.L. Richards, and No-H. Yeh, Jo Appl. Phys. 48, 4865 (1977)。

7. J. Clarke, GoI. Hoffer, and P.L. Richards, Rev, Phys, Appl. 9, 
$69(1974)$.

8. N.S.Nishioka, P.L. Richards, and D.P. Woody (unpublished).

9. E.H. Putly, Phys, Tech. 4, 202 (1973).

10. P.R. Bratt and $N \cdot N$. Lewis, "Development of doped-germanium photoconductors fox astronomical observations at wavelengths from 30 to $120 \mu m, " N A S A=C R-152,046, S B R C$, Nov。 30, 1977.

11. G.E. Stillman, C.M. Wolfe, and J.O. Dimmock, "Semiconductors and Semimetals" (R.K. Willardson and A.C. Beer, eds.) Vol. 12, pp. 169-290, Academic Press, New York, 1977.

12. A.G. Kazanskii, P.L. Richards, and E.E.Haller, Appl: Phys. Lett. $31,496(1977)$.

13. P.L. Richards and M.R. Hueschen (private communication).

14. M.A. Kinch, Appl. Phys, Lett. 12, 78 (1968).

15. B. Paul and H. Welss, Solid St, Electron. 11,979 (1968).

16. H.J. Goldsmid, N. Savvides, and C. Uher, Jo Phys, D 5, 1352 (1972)。

17. E.R. Washwe11, S.R. Hawkins, and K.F. Cuff, Appl. Phys . Lett. $17.164(1970)$.

18. R.A. Smith, F。E. Jones, and R.P. Chasmar, The Detection and Measurements of Infrared Radiation, 2nd ed. (Oxford U.P., Oxford 1968).

19. See, for example, F.N.H. Robinson, Noise and Fluctuations in Electronic Devices an Circuits (Clarendon, Oxford, 1974).

20. Ma Strongin, $0 . F_{*}$ Kammerer, and A. Paskin, Phys, Rev, Lett. 14, $949(1965)$

21. D.E. Prober, Rev, Sci. Inst, 45, 848 (1974).

22. R.F. Voss, and J. Clarke, Phys. Rev, B 13, 556 (1976). 
23. M. Born and E. Wolf, Principles of Optics, 5th ed. (Pergamon, Oxford, 1975), pp. 628-632.

24. R.C. OhImann, P.L. Richards, and Mo TInkham, J. Opt. Soc. Am. $48,531(1958)$.

25. E.V. Loewenstein, D.R. Smith, and R.L.Morgan, Appl. Opt. 12, $398(1973)$.

26. W.S. Boyle and A.D. Brailsford, Phys. Rev, 120, 1943 (1960).

27. M.B. Ketchen and J. Clarke, Phys, Rev。B 17, 114 (1978)。

28. J.W. Eberhard and P.M. Horn, Phys. Reve Lett. 39, 643 (1977)。

29. J. Clarke, W.M. Goubau, and M.B. Ketchen, Jo Low Temp. Phys. 25, $99(1976)$.

30. D.W. Pashley, Advan。 Phys. 14, 361 (1965).

31. K.L. Chopra, "Thin Film Phenomena", pp. 137-254, New York, McGrawHi11 (1969)。

32. L.I. Maisse1 and R. Glang, "Handbook of Thin Film Technology", pp. 8-3-32, New York McGraw-Hil1 (1970).

33. B.W. Sloope and C.0. Tiller, Appl. Phys, Lett, 8, 223 (1966);

J.A. Koutsky, A.G. Walton, and E. Baer, J. Polymer Sci. 4,611 (1966).

34. Lo Brueck, Ann. Physik 26, 233 (1936).

35. H. Holloway and L.C. Bobb, J. Appl. Phys, 38, 2893 (1967).

36. C.D. Tesche and J. Clarke, J. Low Temp. Phys. 29, 301 (1977).

37. G.I. Rochlin, Rev, Sc1. Instrum, 41, 73 (1970)。

38. J.D.N. Cheeke, Jo de Physique 31, Supp1。C3, 129 (1970).

39. P. Herth and 0 , Weis, $Z$. Angew. Phys, 29, 101 (1970).

40. O. Weis, Jo de Phystque 33, Supp1. C4, 48 (1972)。 
41. W.A. Little, Can。 Jo Phys. 37, 334 (1959)。

42. R. Berman and D.K.C. MacDonald, Proc. Roy. Soc. A 209. 368 (1951);

A $211,122(1952)$.

43. R.J. Be11, "Introductory Fourier Transform Spectroscopy", pp. 69-77, New York, Academic Press (1972). 
Table 1. Measurement conditions for detectors given in Fig. 1.

\begin{tabular}{lccc} 
Detector & Area $\left(\mathrm{mm}^{2}\right)$ & $\begin{array}{c}\text { Operating } \\
\text { temperature }(\mathrm{K})\end{array}$ & References \\
\hline $\begin{array}{l}\text { (a) Composite Al } \\
\text { superconducting } \\
\text { boloneter }\end{array}$ & 16 & 1.27 & 5,6 \\
(b) Composite SNS & 32 & 1.4 & 6,7 \\
bolometer & 16 & 1.2 & 8 \\
(c) Composite Ge:Ga & & 1.2 & 14 \\
bolometer & 2 & 4.2 & 11 \\
(d) InSb $\mathrm{GaAs}$ & 4 & 2.0 & 12,13 \\
(f) Ge:Ga (stressed) & 6 & 3 & 10 \\
(g) Ge:Ga & 0.75 & 3 & 10 \\
(h) Ge:Be & 0.75 & &
\end{tabular}

Table 2. Parameters of five transition edge bolometers

\begin{tabular}{|c|c|c|c|c|c|}
\hline Bolometer & 1 & 2 & 3 & 4 & 5 \\
\hline $\begin{array}{l}\text { Dimensions } \\
(\mathrm{mm})\end{array}$ & $2 \times 4 \times 0.135$ & $2 x 4 x 0.135$ & $2 \times 4 \times 0.135$ & $2 \times 4 \times 0.135$ & $4 \times 4 \times 0.050$ \\
\hline $\mathrm{T}(\mathrm{K})$ & 1.30 & 1.28 & 1.40 & 1.28 & 1.27 \\
\hline $\mathrm{dR} / \mathrm{dT}\left(\Omega \mathrm{K}^{-1}\right)$ & $60^{a}$ & $80^{2}$ & 2,000 & 800 & 200 \\
\hline$G\left(10^{-8} W K^{-1}\right)$ & 2.5 & 4 & 3.5 & 3.5 & 2 \\
\hline$\tau(s)$ & 0.1 & 0.05 & 0.05 & 0.05 & 0.08 \\
\hline$I(\mu \mathrm{A} x \mathrm{~ms})$ & 14 & 11 & 0.7 & 0.7 & 5 \\
\hline $\begin{array}{l}\text { NEP }(5, \mathrm{~Hz}) \\
\quad\left(10^{-15} \mathrm{WHz}^{-1 / 2}\right)\end{array}$ & 5 & 3 & 3 & 3 & 2 \\
\hline
\end{tabular}

${ }^{a}$ Al films did not have cut edges. 
Table 3. Calculated heat capacities at $1.27 \mathrm{~K}$ of components of bolometex 5

\begin{tabular}{lcc}
\hline Component & Dimensions $(\mathrm{mm})$ & $\begin{array}{c}\text { Heat capacity } \\
\left(10^{-10} \mathrm{JK}^{-1}\right)\end{array}$ \\
\hline $\begin{array}{l}\text { Sapphire } \\
\text { In films (4) }\end{array}$ & $0.5 \times 4 \times 0.050$ & 6.6 \\
Bi heater & $4 \times 0.5 \times 0.000088$ & 2.8 \\
Al thermometer & $4 \times 0.18 \times 0.000070$ & 0.2 \\
Bi absorbex & $4 \times 4 \times 0.000093$ & 0.1 \\
& 4.8 & 11.5 \\
\hline
\end{tabular}


Table 4. Measured parameters of bolometer 5

\begin{tabular}{|c|c|}
\hline Operating temperature & $1.27 \mathrm{~K}$ \\
\hline G & $2.0 \times 10^{-8} \mathrm{WK}^{-1}$ \\
\hline$\tau$ & $60 \mathrm{~ms}$ \\
\hline $\mathrm{C}$ & $1.2 \times 10^{-9} \mathrm{JK}^{-1}$ \\
\hline $\mathrm{dR} / \mathrm{dT}$ & $2008 K^{-1}$ \\
\hline I & $5.3 \mu \mathrm{A} r \mathrm{~ms}$ \\
\hline$G_{e}$ & $1.44 \times 10^{-8} \mathrm{WK}^{-1}$ \\
\hline$\tau^{\tau} \mathrm{e}$ & $83 \mathrm{~ms}$ \\
\hline$S=(\partial V / \partial T) / G_{e} \quad(2 \mathrm{~Hz})$ & $7.4 \times 10^{4} \mathrm{VW}^{-1}$ \\
\hline$\tau_{m}$ & $25 \mathrm{~s}$ \\
\hline$\alpha$ & 70 \\
\hline NEP measured & $(1.7 \pm 0.1) \times 10^{-15} \mathrm{WHz}^{-1 / 2}$ at $2 \mathrm{H}$ \\
\hline$(\mathrm{NEP})_{\mathrm{Th}}$ & $1.3 \times 10^{-15} \mathrm{WHz}^{-1 / 2}$ \\
\hline Dynamic range in $1 \mathrm{~Hz}$ bandwidth & $10^{4}$ \\
\hline Power spectrum of bath fluctuations & $1.6 \times 10^{-12}\left(1 \mathrm{~Hz}^{2} / \mathrm{f}^{2}\right) \mathrm{K}^{2} \mathrm{~Hz}^{-1}$ \\
\hline Effective absorptivity, $\varepsilon_{e}$ & $0.47 \pm 0.05$ \\
\hline$D^{*}$ & $(1.1 \pm 0.1) \times 10^{14} \mathrm{cmW}^{-1} \mathrm{~Hz}^{1 / 2}$ \\
\hline
\end{tabular}


Table 5. Heat capacity of several materials used for composite bolometer

\begin{tabular}{|c|c|c|c|c|c|c|c|c|c|}
\hline \multicolumn{2}{|c|}{ Material } & \multicolumn{2}{|c|}{ Sapphire } & \multicolumn{2}{|c|}{ Diamond } & \multicolumn{2}{|c|}{ Bismuth } & \multicolumn{2}{|c|}{ Chromium } \\
\hline \multicolumn{2}{|c|}{ Dimension (mm) } & \multicolumn{2}{|c|}{$4 \times 4 \times 0.05$} & \multicolumn{2}{|c|}{$4 \times 4 \times 0.05$} & \multicolumn{2}{|c|}{$4 \times 4 \times 0.000093$} & \multicolumn{2}{|c|}{$4 \times 4 \times 0.00001$} \\
\hline Temper & rature $(K)$ & 1.27 & 0.4 & 1.27 & 0.4 & 1.27 & 0.4 & 1.27 & 0.4 \\
\hline $\begin{array}{ll}\hat{H} & \\
0 & -1\end{array}$ & Electronic & - & - & - & - & 0.007 & 0.002 & 0.39 & 0.12 \\
\hline 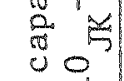 & Phonon & 6.6 & .21 & .84 & .026 & 1.7 & 0.051 & 0.0035 & 0.000 \\
\hline 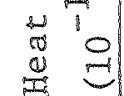 & Total & 6.6 & .21 & .84 & .026 & 1.7 & 0.053 & 0.39 & 0.12 \\
\hline
\end{tabular}


FIGURE CAPTIONS

1. Performance of the far-infrared detectors. D* is plotted vs. $\lambda$ for all the detectors except for (d) and (f) where D is plotted vs. $\lambda$ 。

2. Configuration of superconducting transition edge bolomerer and mount.

3. Schematic of electronics for superconducting transition edge bolometer.

4. Calculated relative response $(\Delta T)^{2}$ of bolometer vs. frequency for perturbations (a) $\left(\Delta \mathrm{P}_{\mathrm{B}}\right)^{2} \cos ^{2}\left(w t+\delta_{1}\right)$ and (b) $\left(\Delta \mathrm{T}_{\mathrm{He}}\right)^{2} \cos ^{2}\left(w t+\delta_{2}\right)$, with $(-\infty)$ and without $(-$ feedback.

5. Measured (-) and calculated (-) responsivity of transition edge bolomter (referred to output of bridge).

6. Measured (0) and calculated (-) noise power spectrum of transition edge bolomter (referred to output of bridge).

7. Measured (-) and calculated (-) NEP of transition edge bolometer.

8. Measured dc resistivity of bismuth films on rough and smooth sapphire surfaces vs。 film thickness.

9. Transmissivity spectra for sapphixe substrates coated with bismuth films of various resistances.

10. Ray traces for a mitered righ angle. Only meridional rays are shown.

11. Configuration for measuring the optical absorptivity of the bolometer.

12. (a) Measured txansmissivity of 2.54-mm-thick Fluorogold ${ }^{\mathrm{TM}}$ Eilter at $1.5 \mathrm{~K}$. (b) Normalized intensity distribution of difference between the blackbody sources at $290 \mathrm{~K}$ and $77 \mathrm{~K}$ viewed through the filter in (a). 
13. Configuration for measuring the optical efficiency of the light pipe.

14. Measured absorptivity vs. input solid angle.

15. Sample configuration for tin noise measurement at the superconducting transition.

16. Scanning electronmicroscope pictures of tin films on sapphire substrates: (a) with evaporation rate $7 \mathrm{~A} / \mathrm{sec}$; (b) with evaporation rate $100 \AA / \mathrm{sec}$.

17. Scanning electronmicroscope pictures of tin films on glass subtrates: (a) with evaporation rate $7 \AA / \mathrm{sec}$; (b) with evaporation rate $100 \AA / \mathrm{sec}$ 。

18. SQUID system used to measure the noise of the tin film. Components within the dashed box are at temepratures below $4.2 \mathrm{~K}$.

19. Schematic diagram of SQUID insert for noise study.

20. Noise power spectrum of the tin film on sapphire substrate. The solid line is the calculated Johnson noise level arising from the standard resistor and the sample.

21. Resistance vs thermal ground temperature for various bias currents.

22. Current voltage characteristics at different thermal ground temperatures.

23. $S_{\mathrm{v}}$ vs. $\mathrm{I}_{1}$ for constant block temperature.

24. Circuit to produce step function changes in the bias current.

25. Test of the computer program used in the decay experiment with an $R-C$ discharge circuit. The solid lines are theoretical curves. The dots (-) and open circles (o) are measured results.

26. Step function response of the tin film on sapphire substrate. The first point of the response curve has been normalized to 
$k \mathrm{r}^{2} / \mathrm{q} \cdot$

27. Cosine transforms of the decay curves obtained from step-function (o) and $\delta$-function (a) perturbations. 


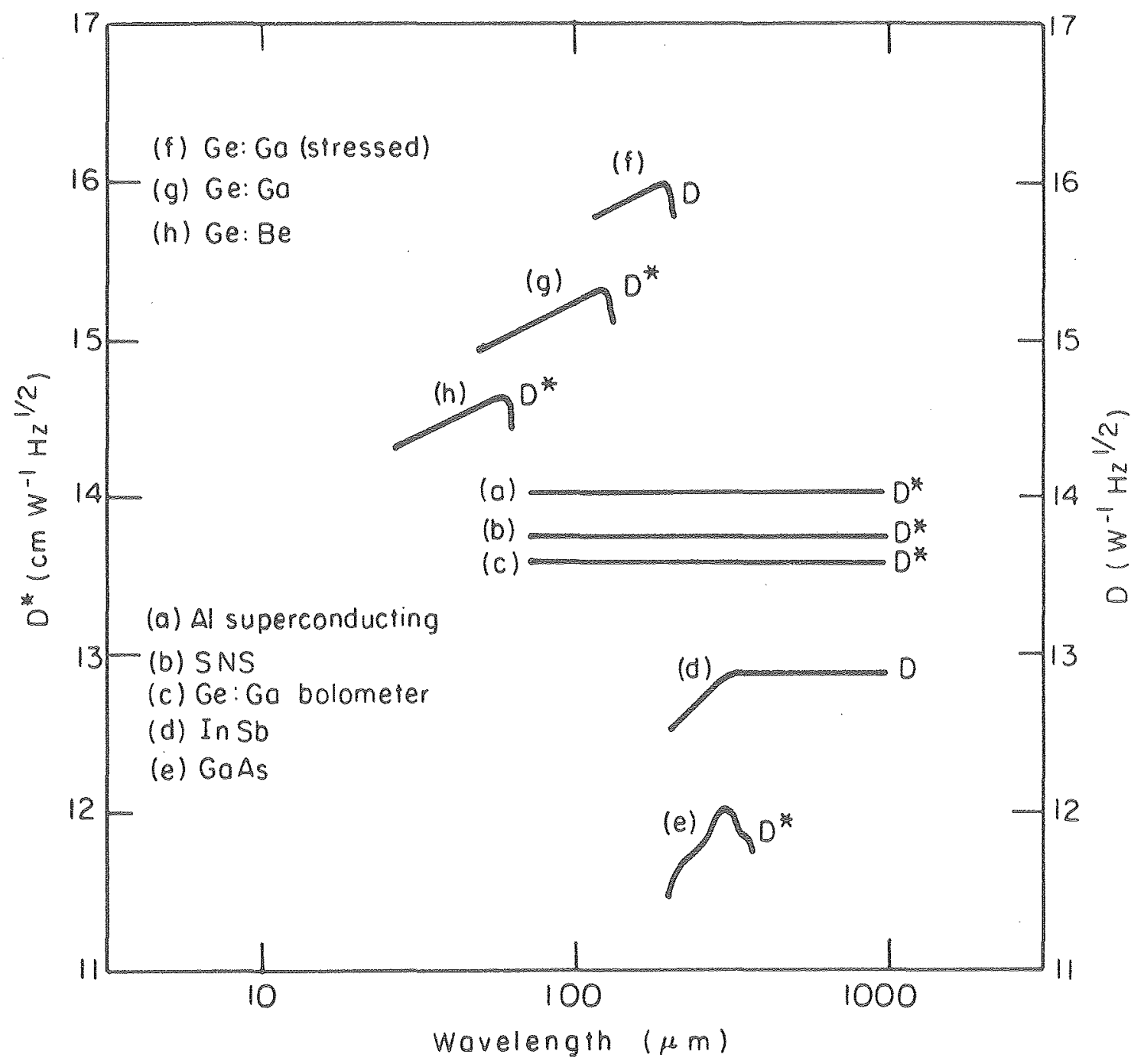

XBL 786-5213

Fig. 1 


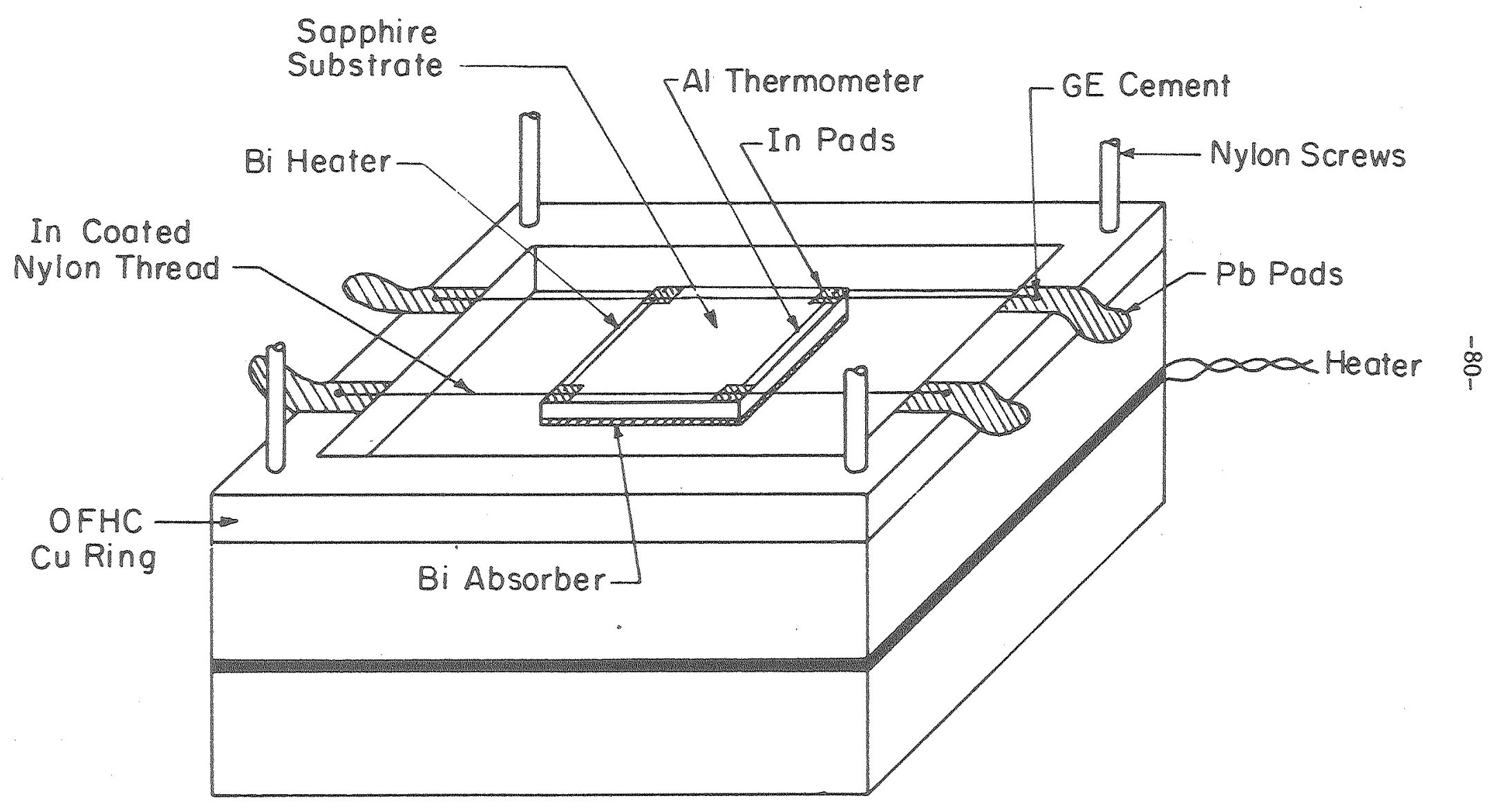

$\times$ BL7612-7932

Fig. 2 


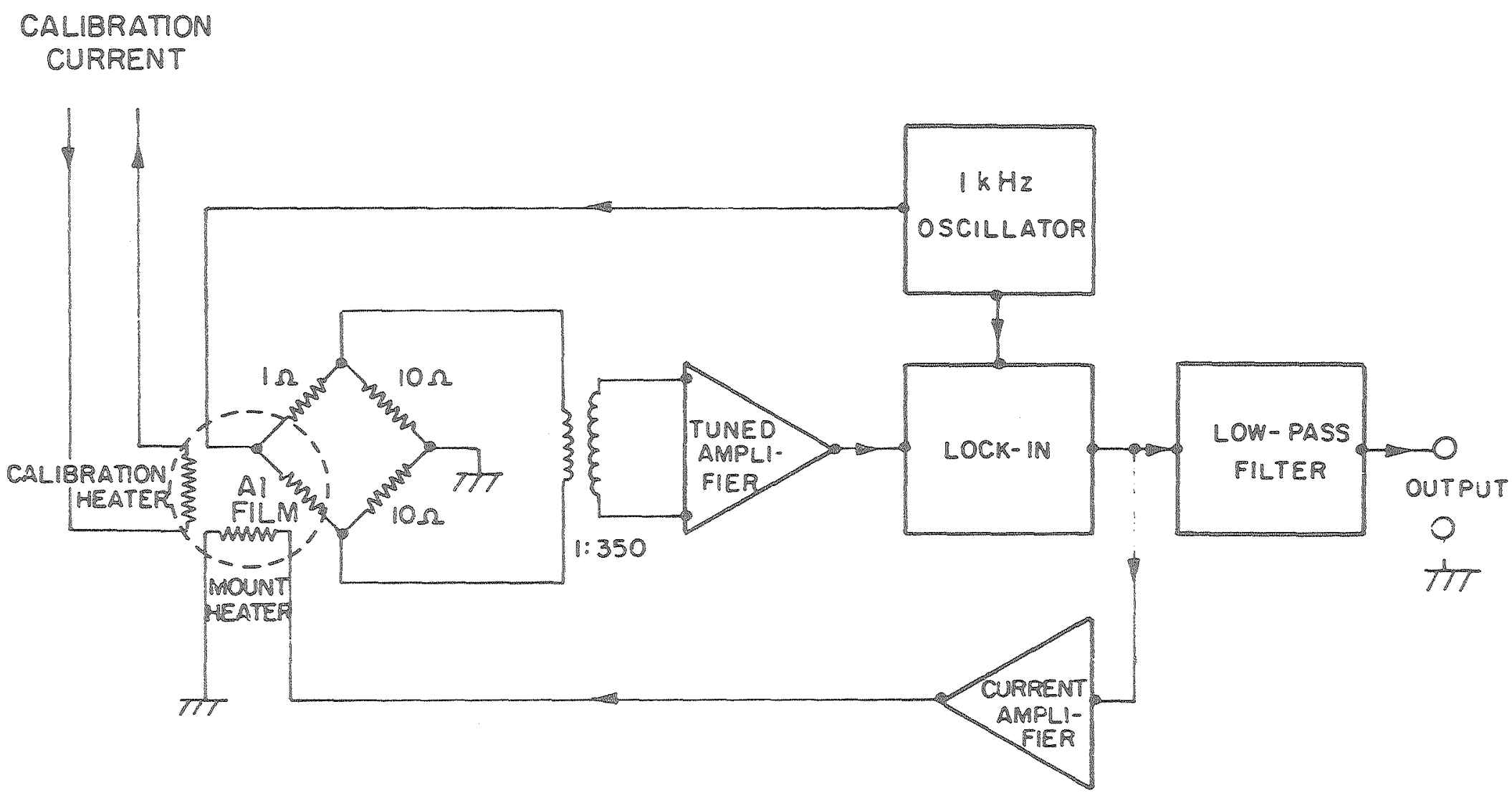




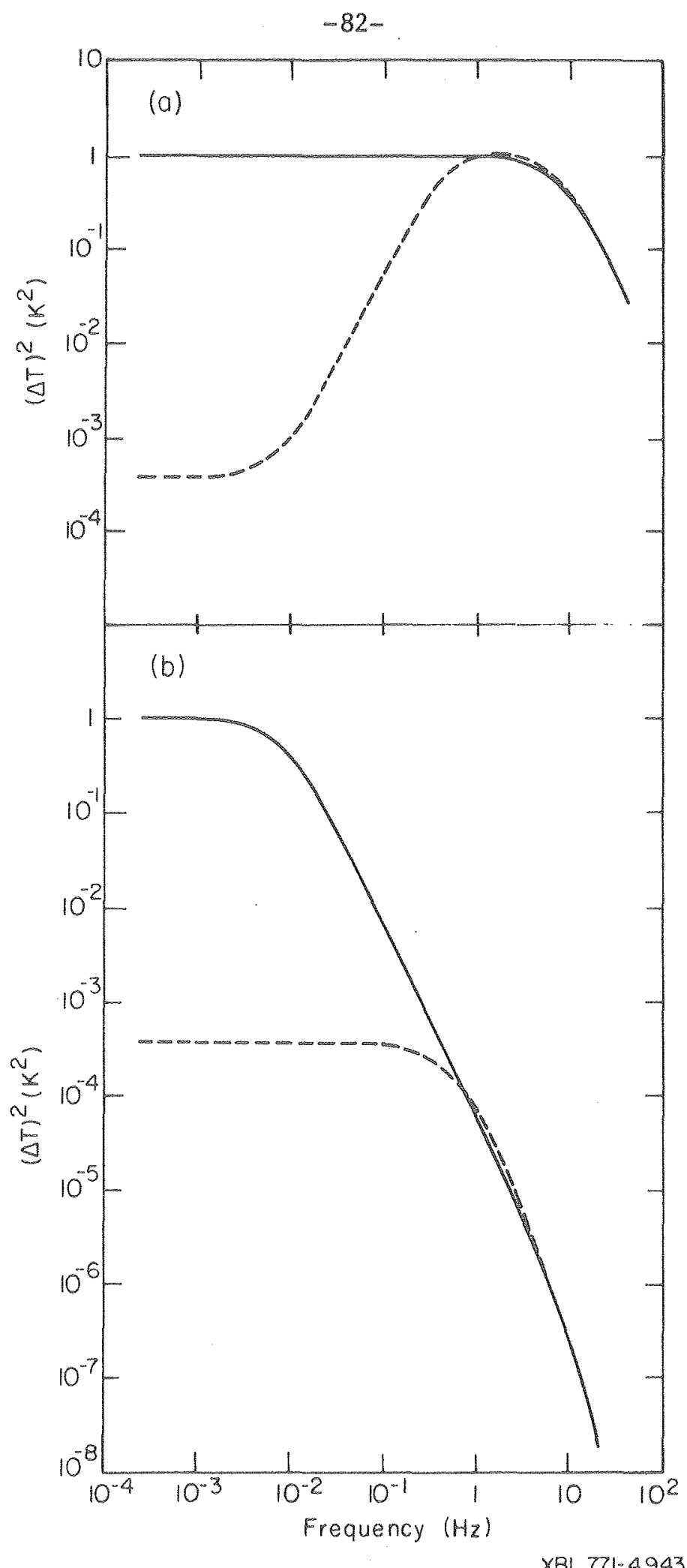

FIg. 4 
$-83-$

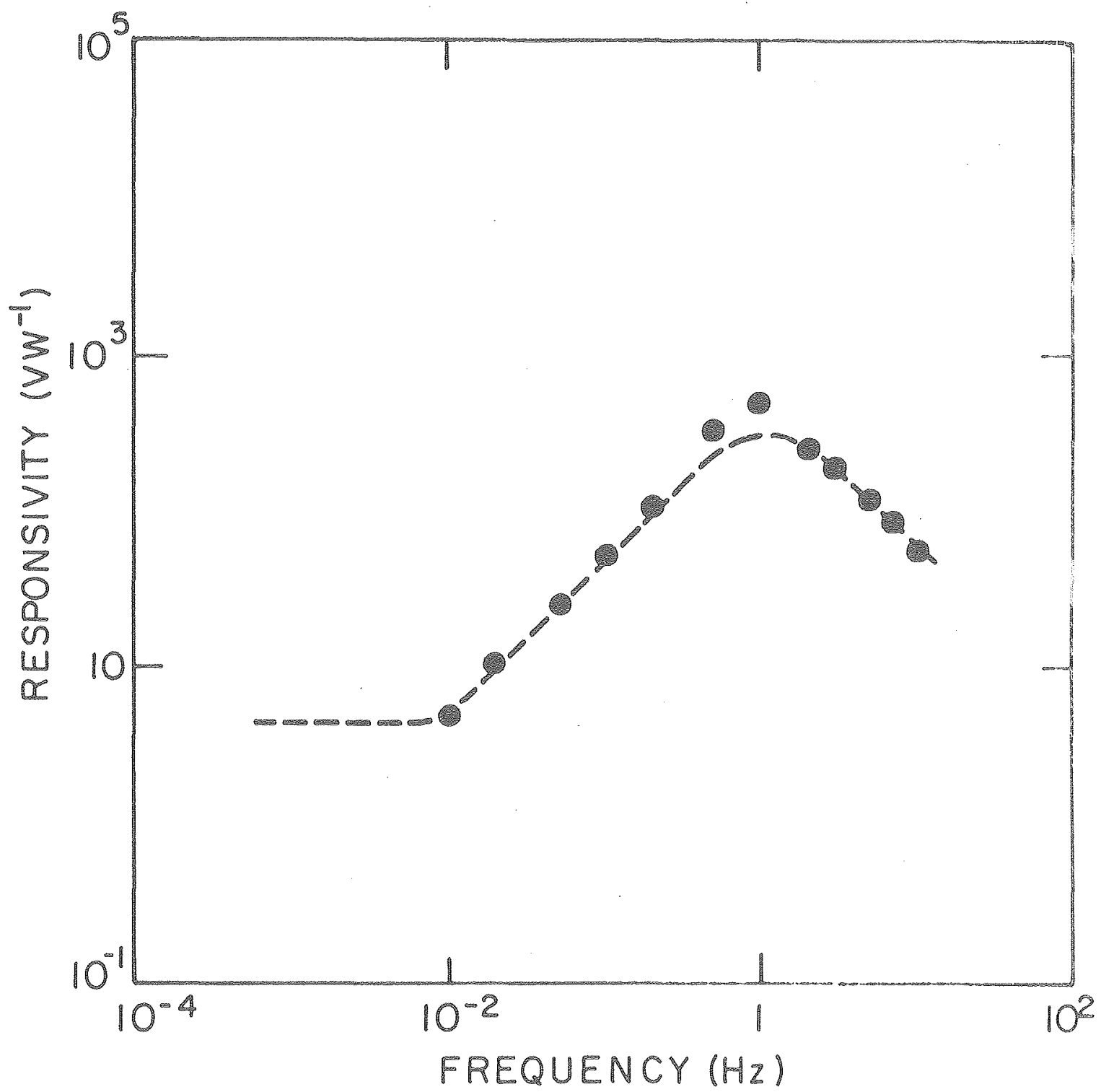

X8L762-7927

Fig. 5 
$-84=$

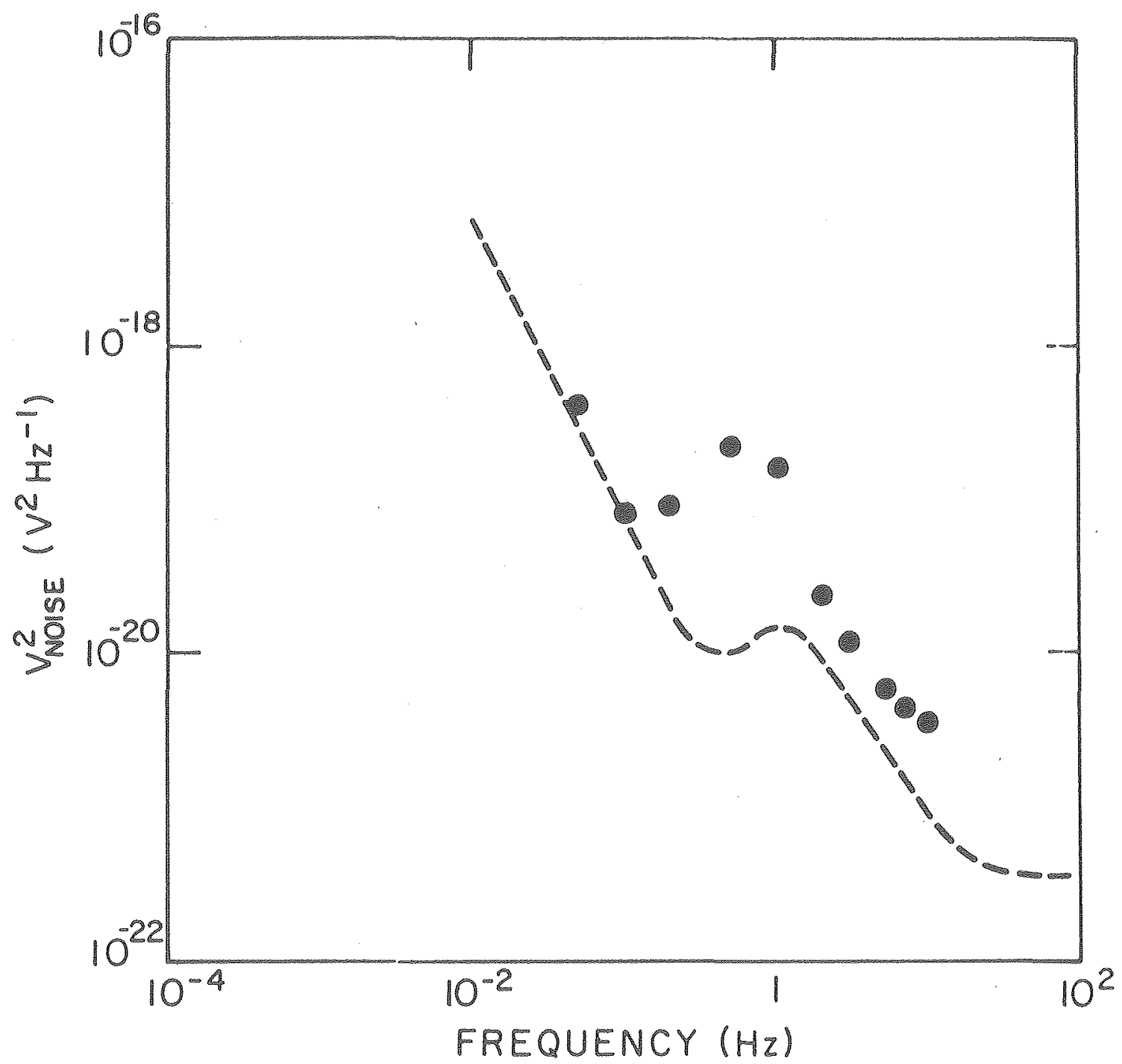

XBL7612-7926

Fig. 6 


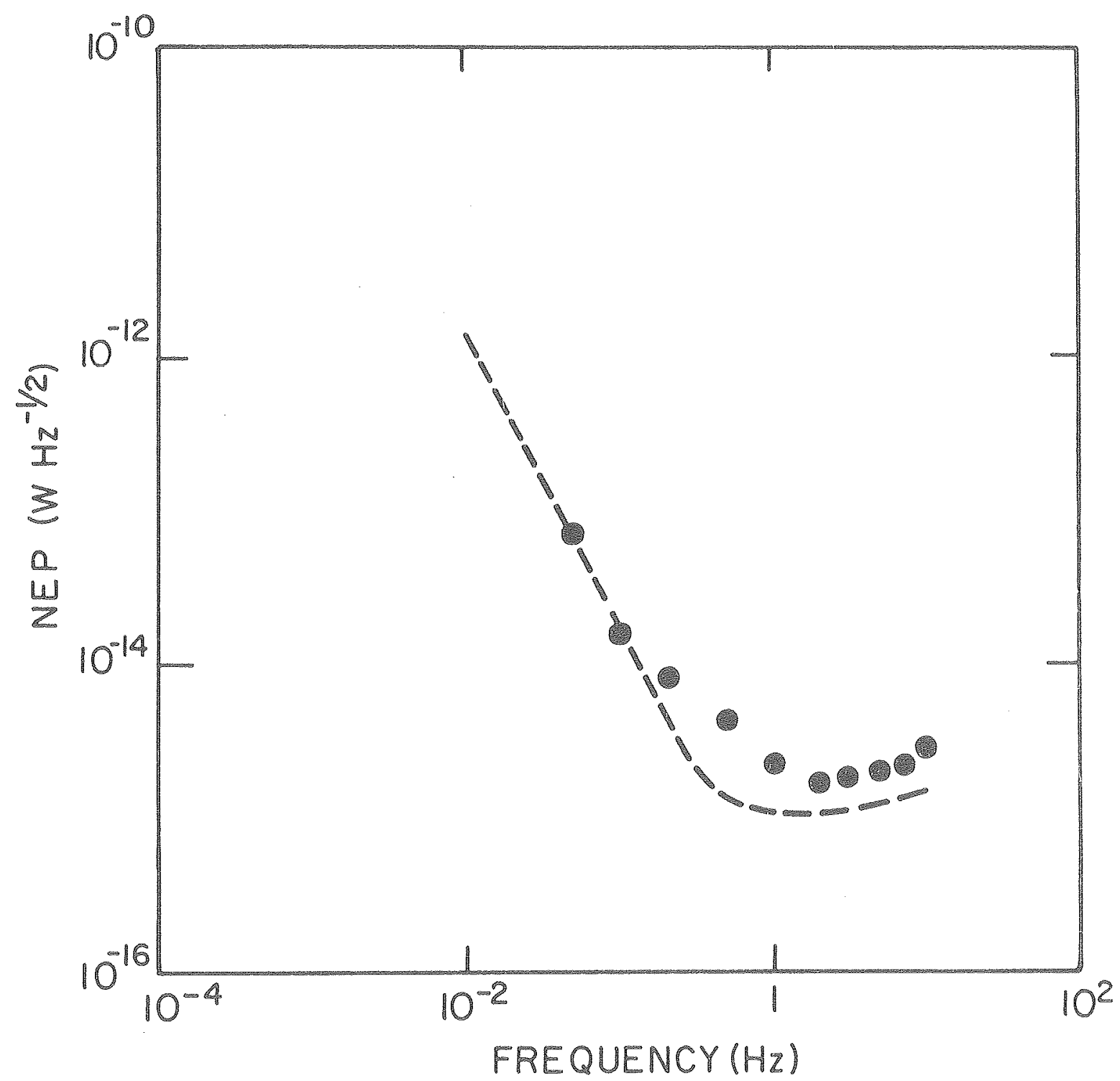

XBL 7612.7925

Fig. 7 
$-86-$

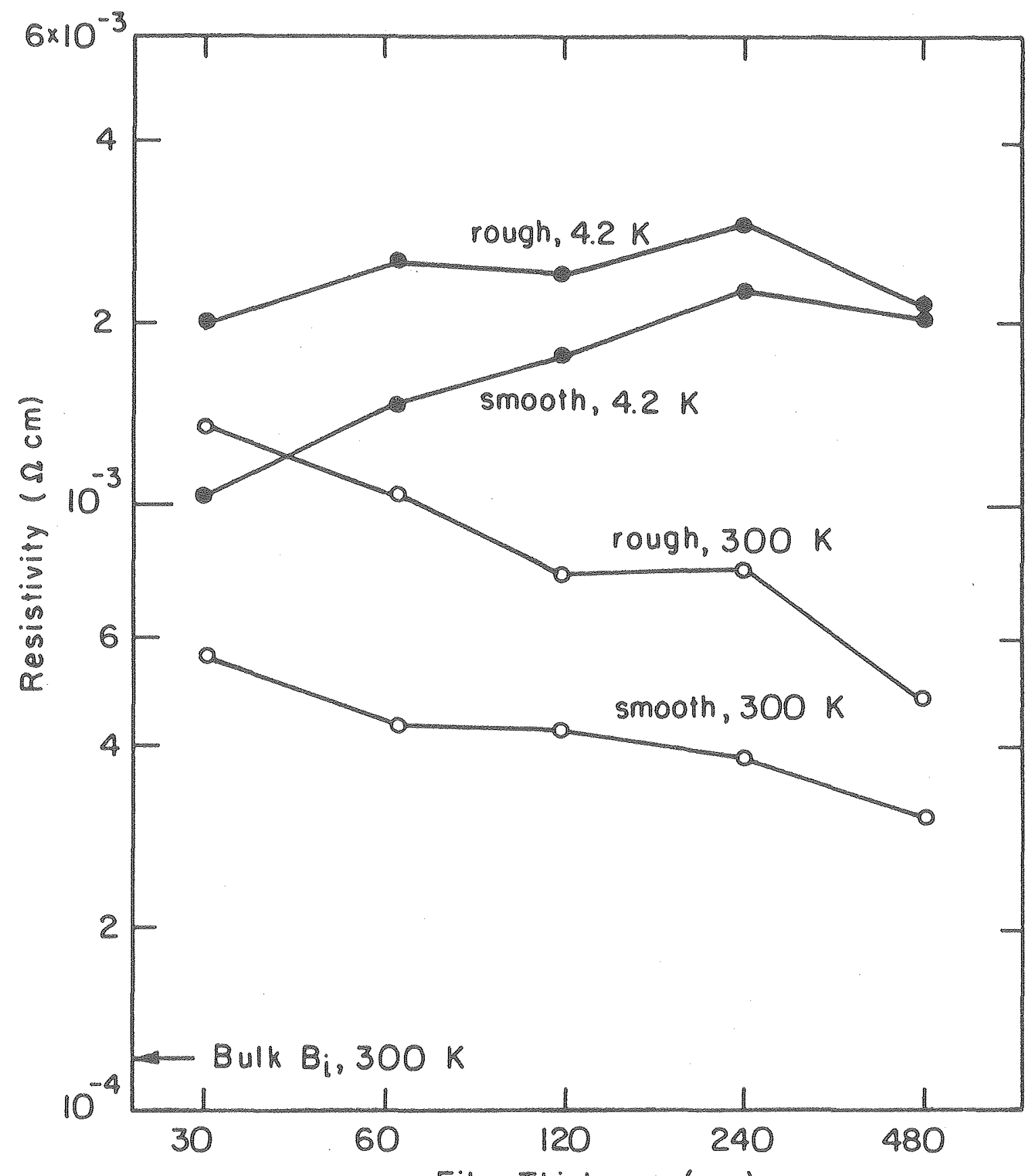

Film Thickness $(\mathrm{nm})$

XBL756-6568A

FIg. 8 


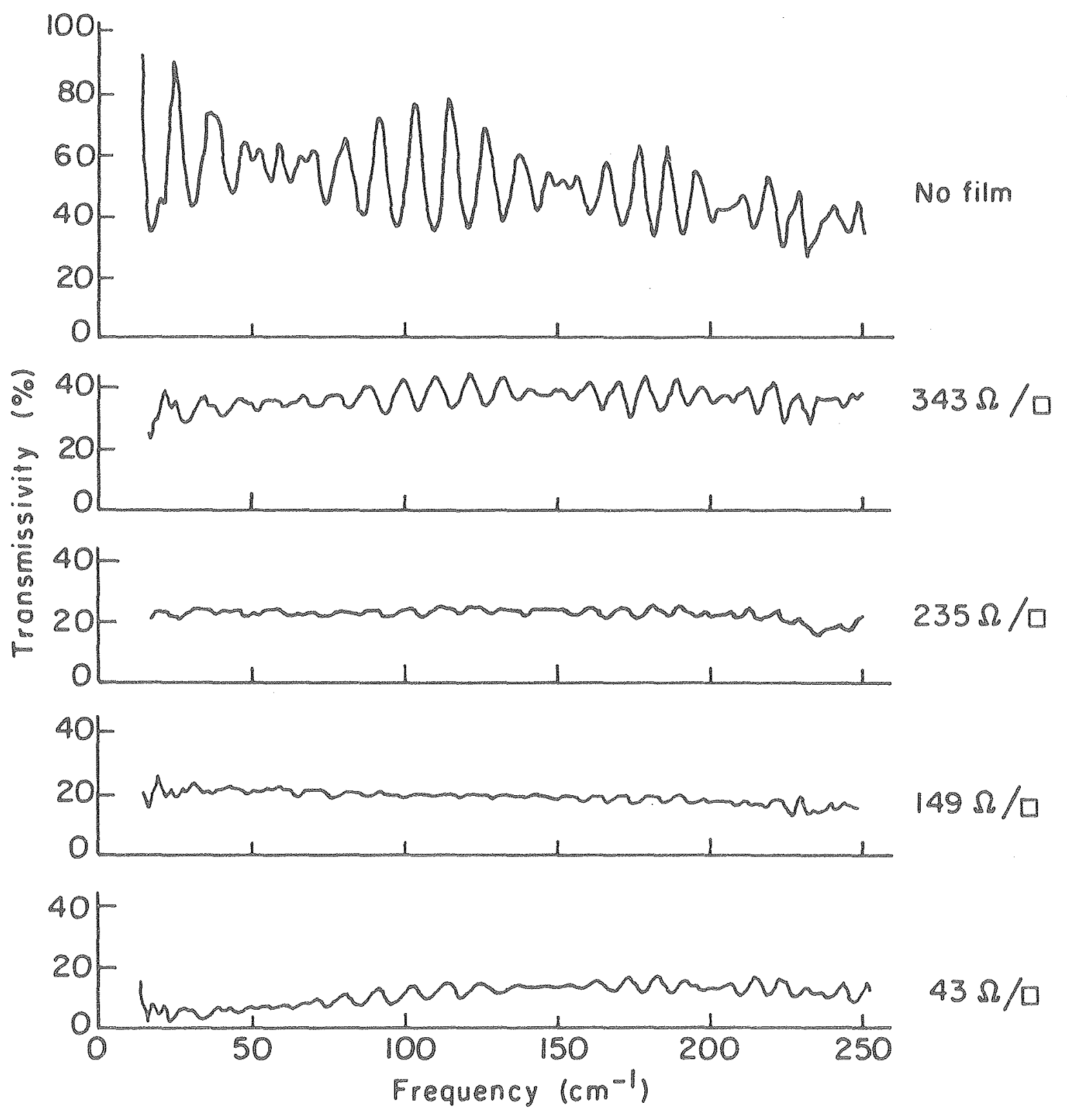

XBL.753-5992

F1g。9 


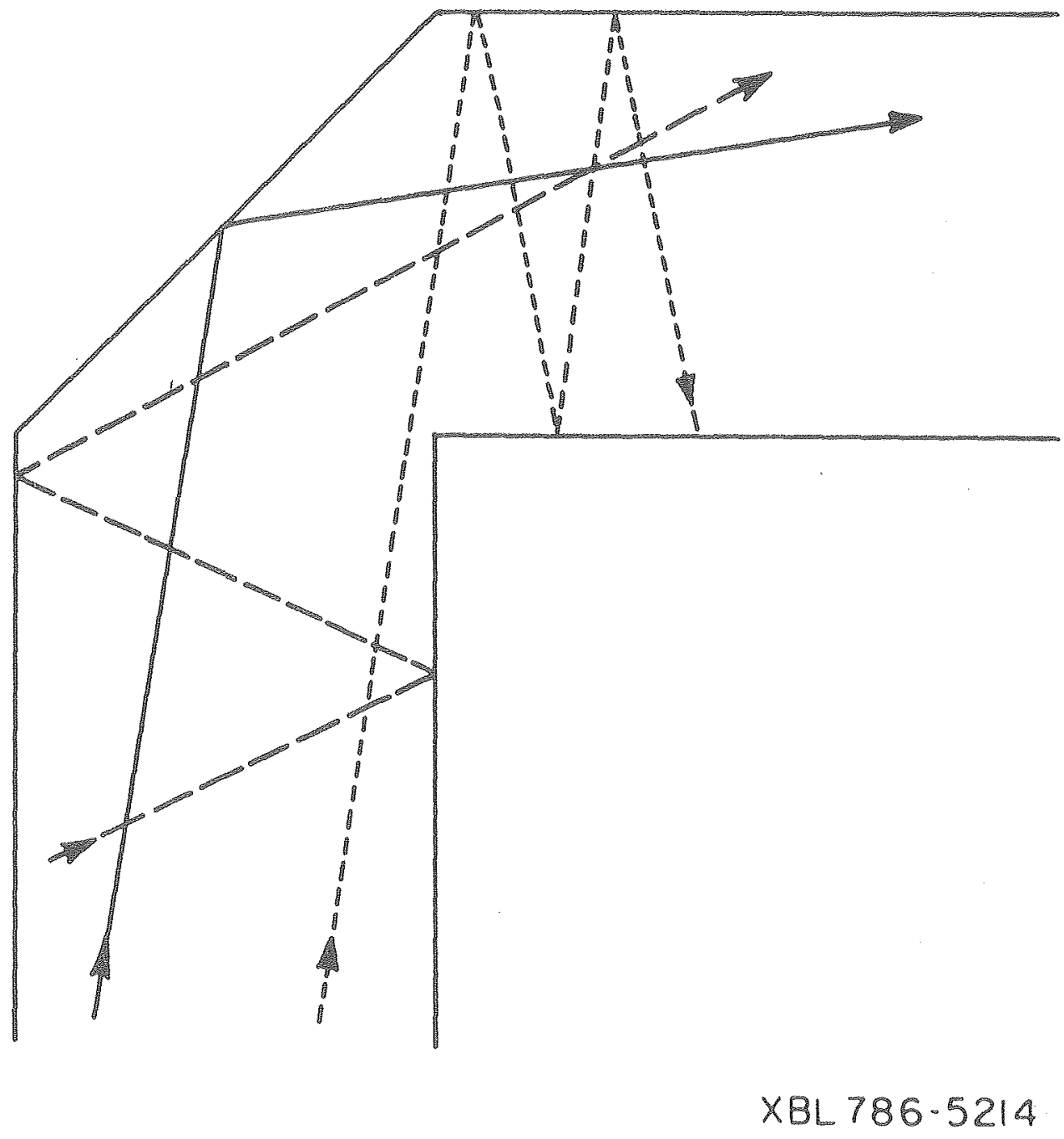

Fig. 10 


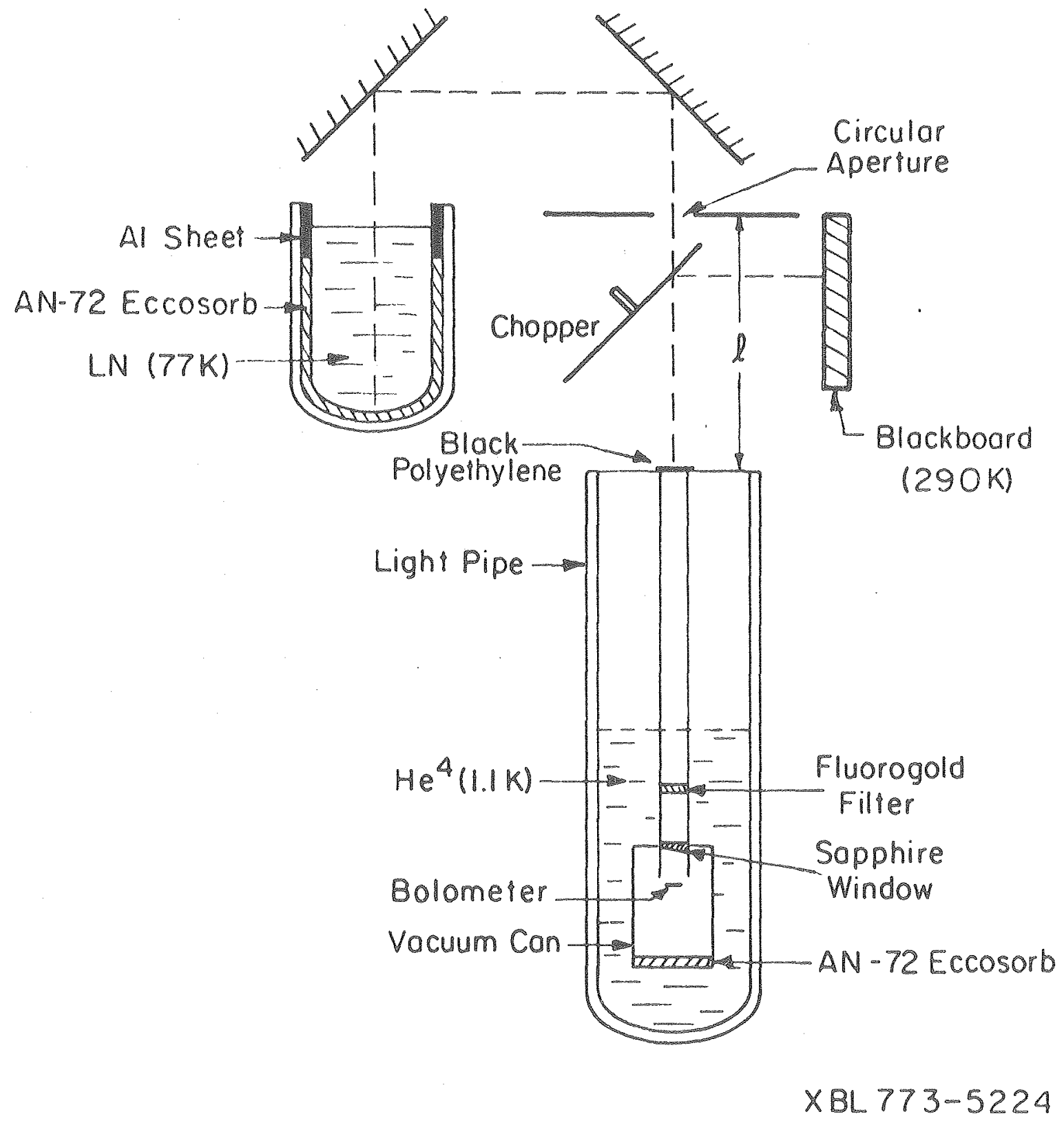

Fig. 11 


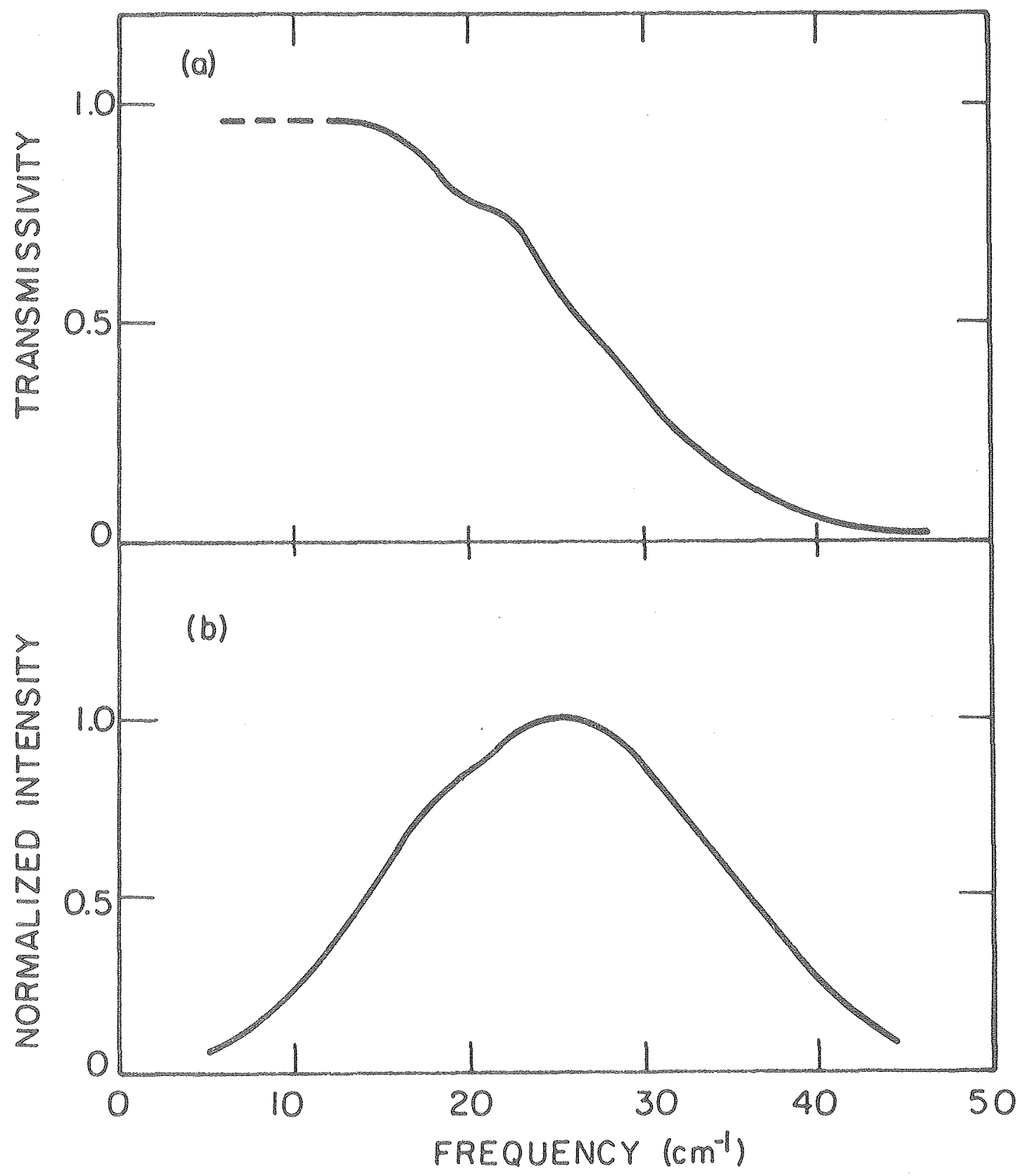

XBL 772-5097

F1g. 12 


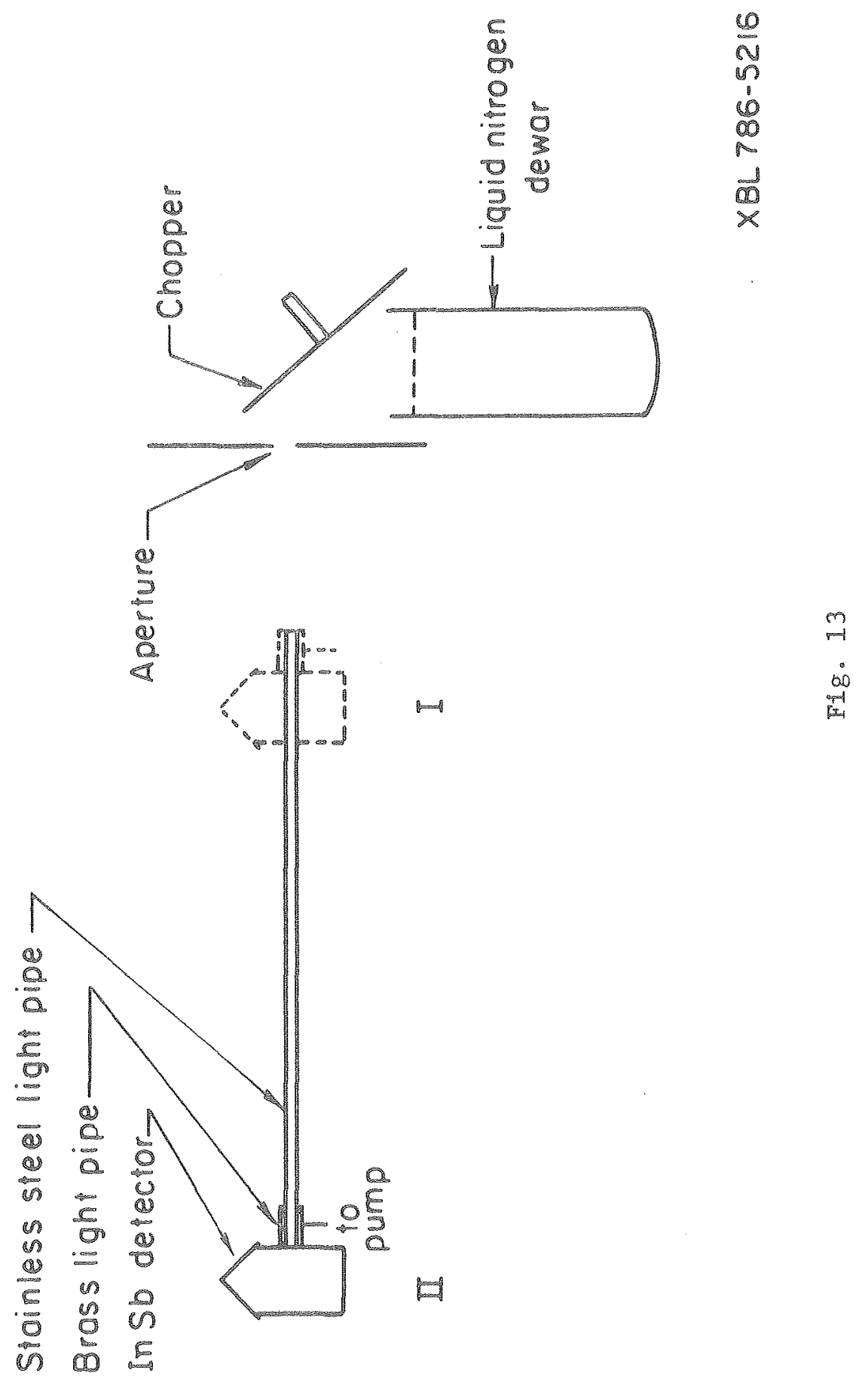









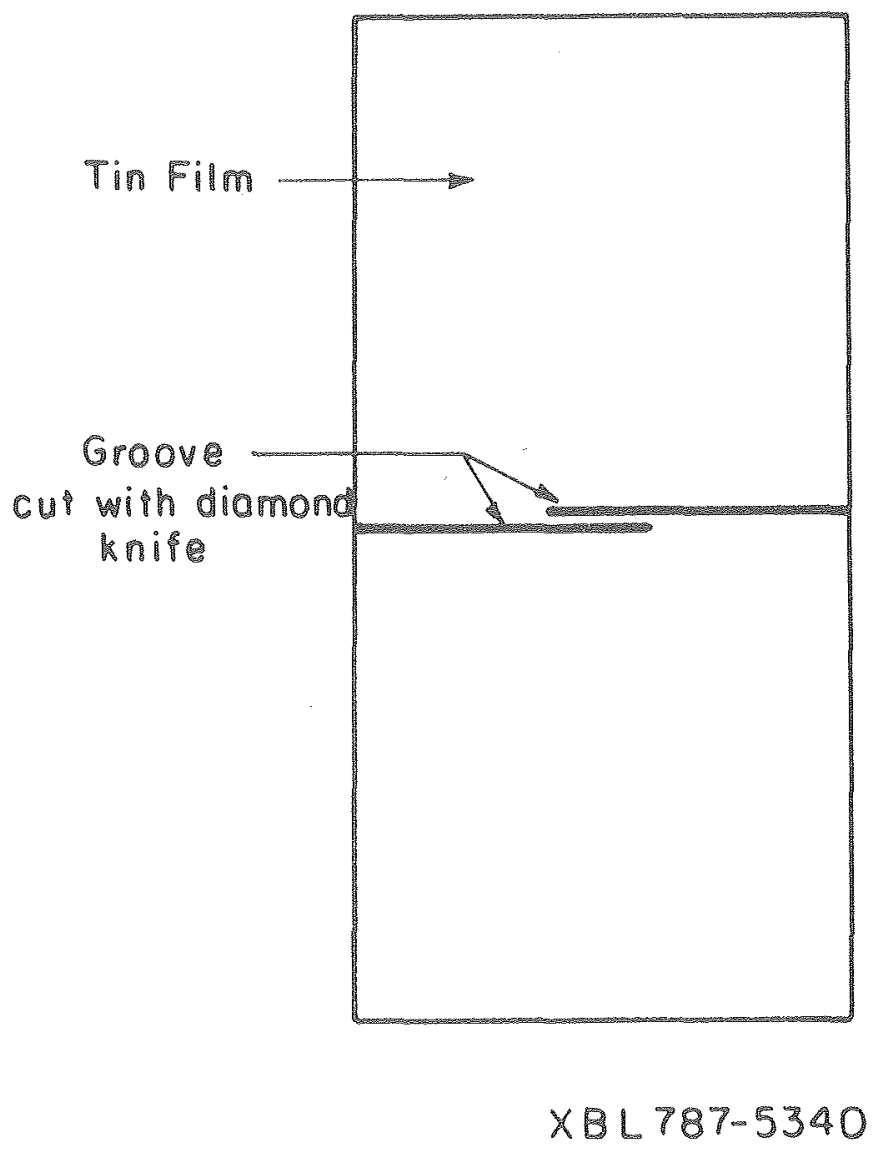

Fig. 15 
(a)

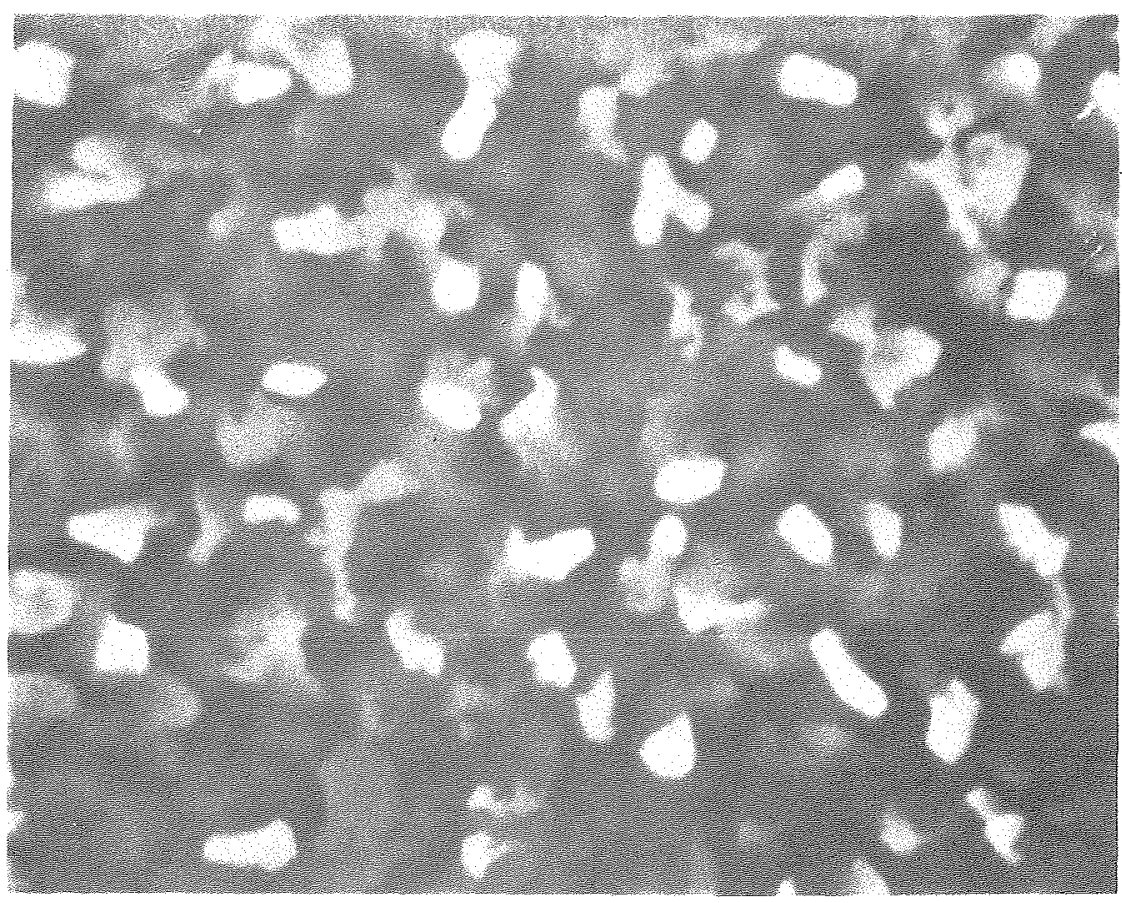

14

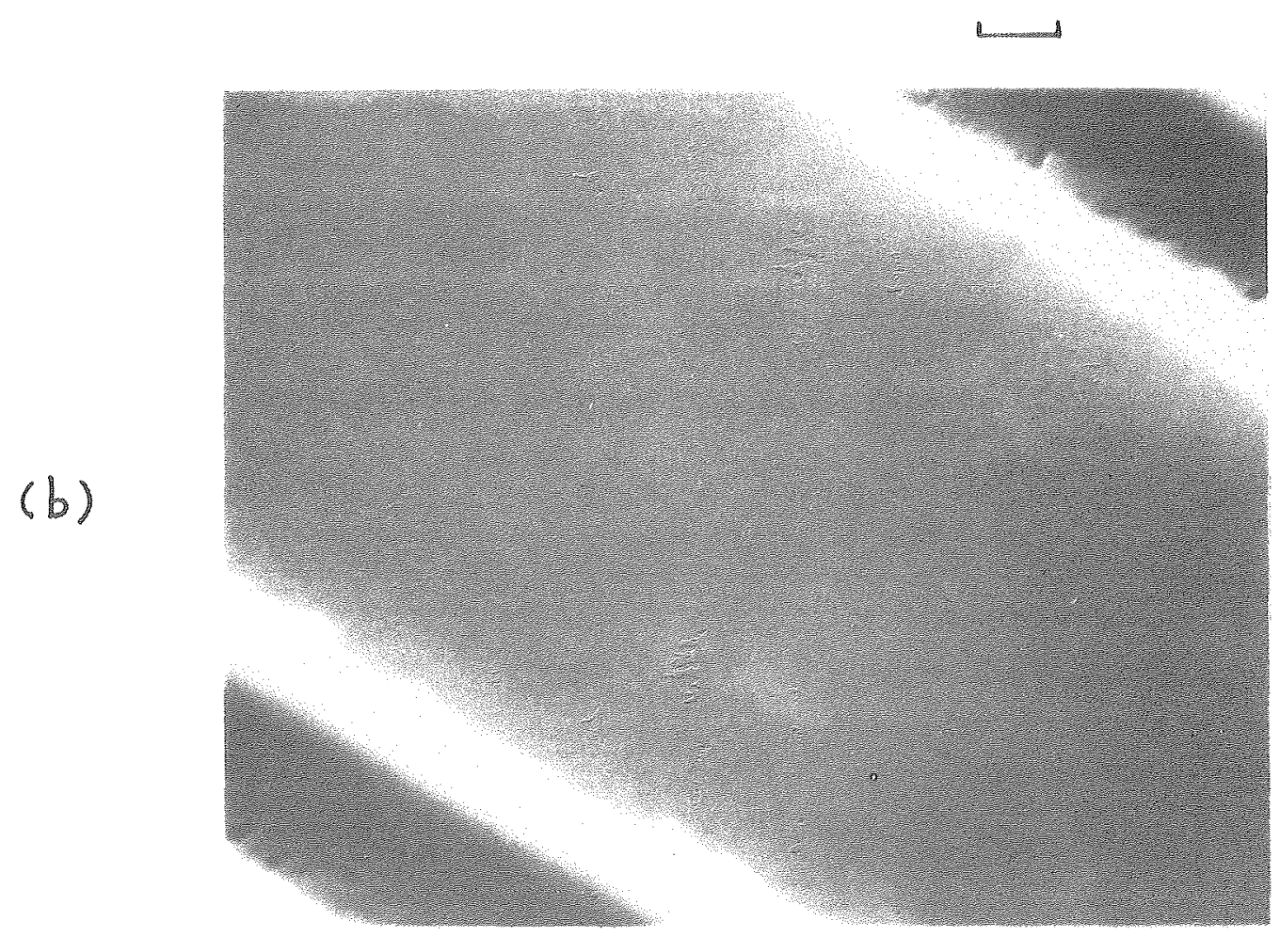

XBB $\quad 787-8346$

Fig. 16 
(a)
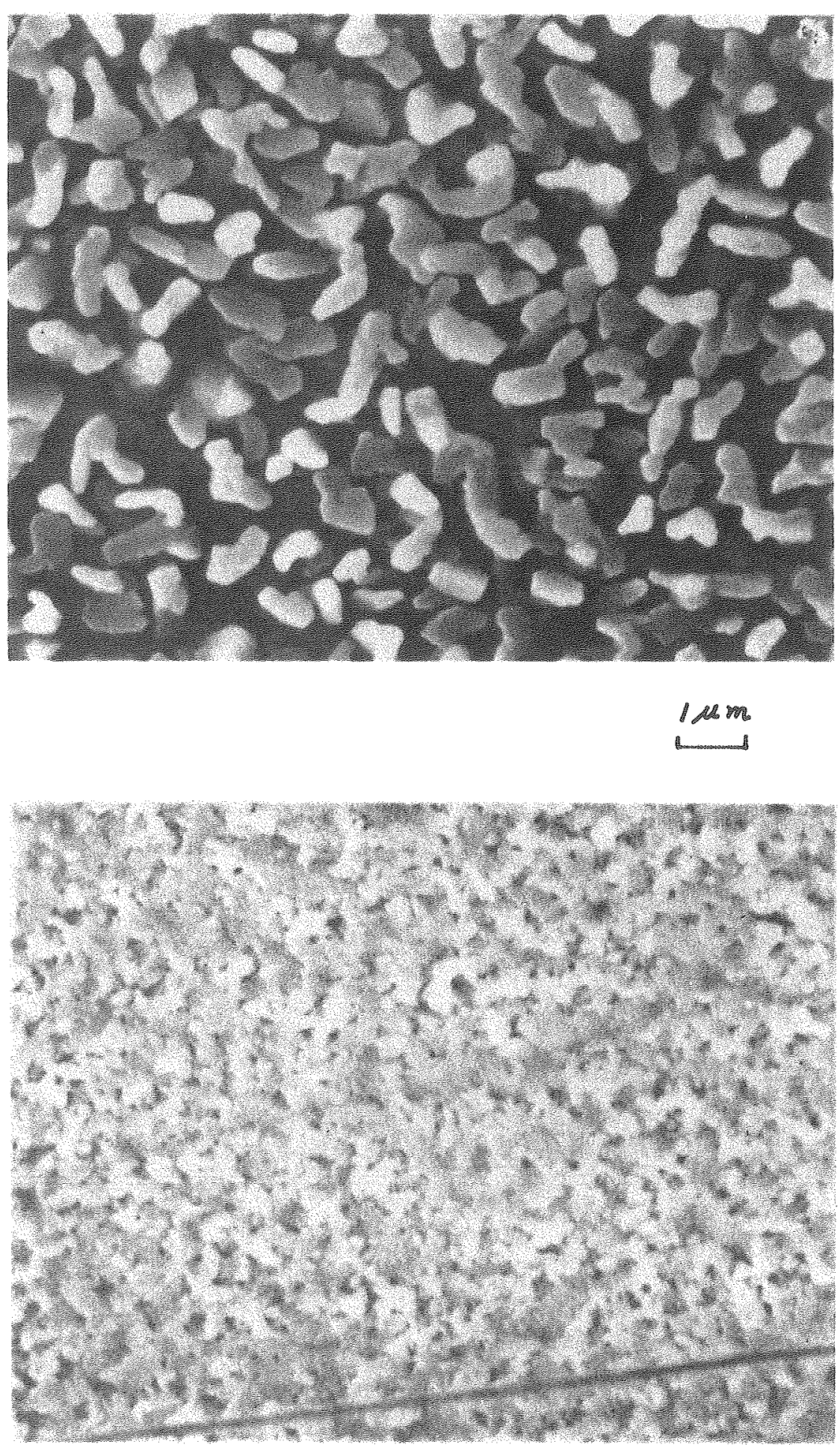

XBB $\quad 787-8347$

Fig. 17 


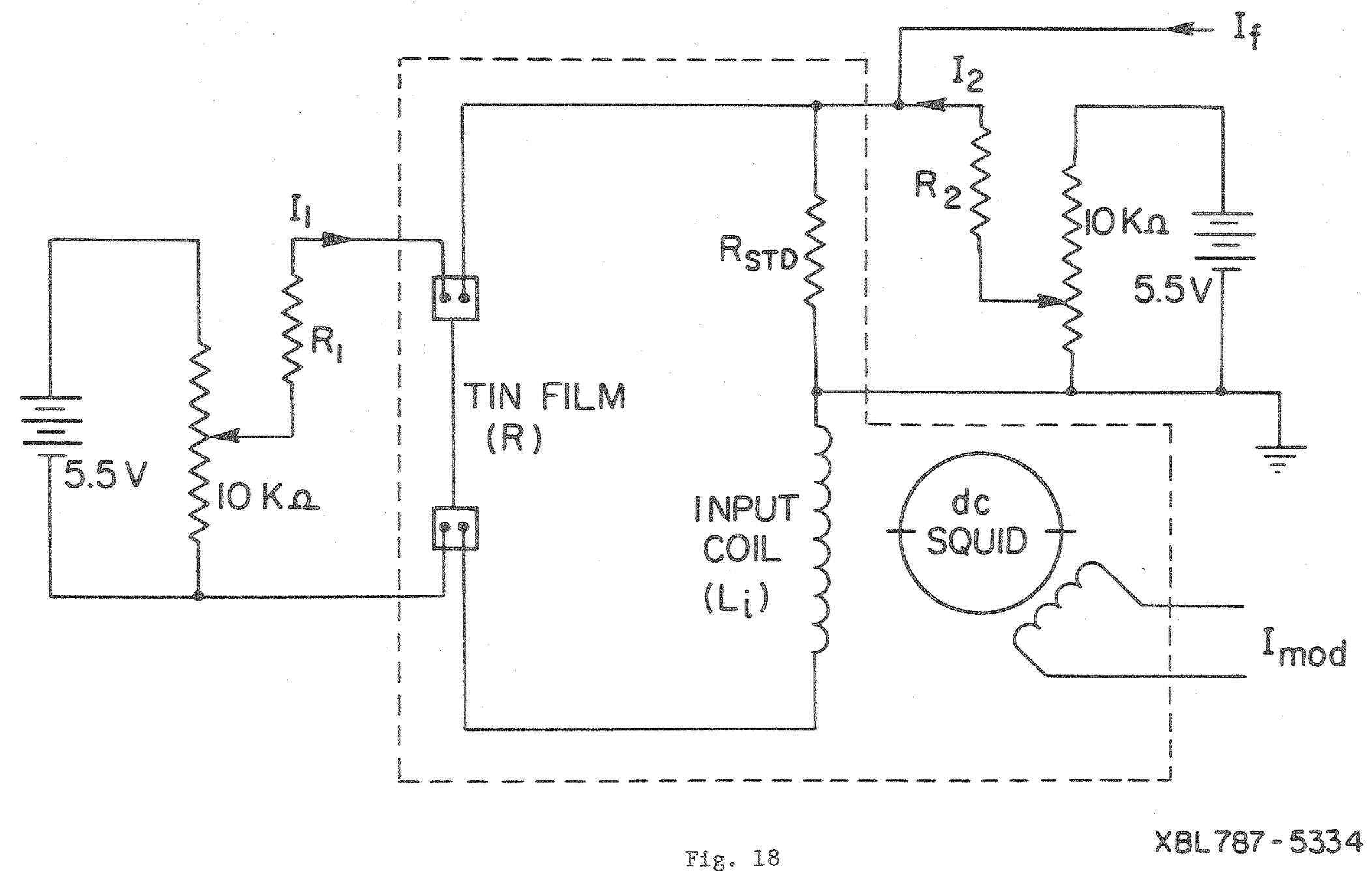



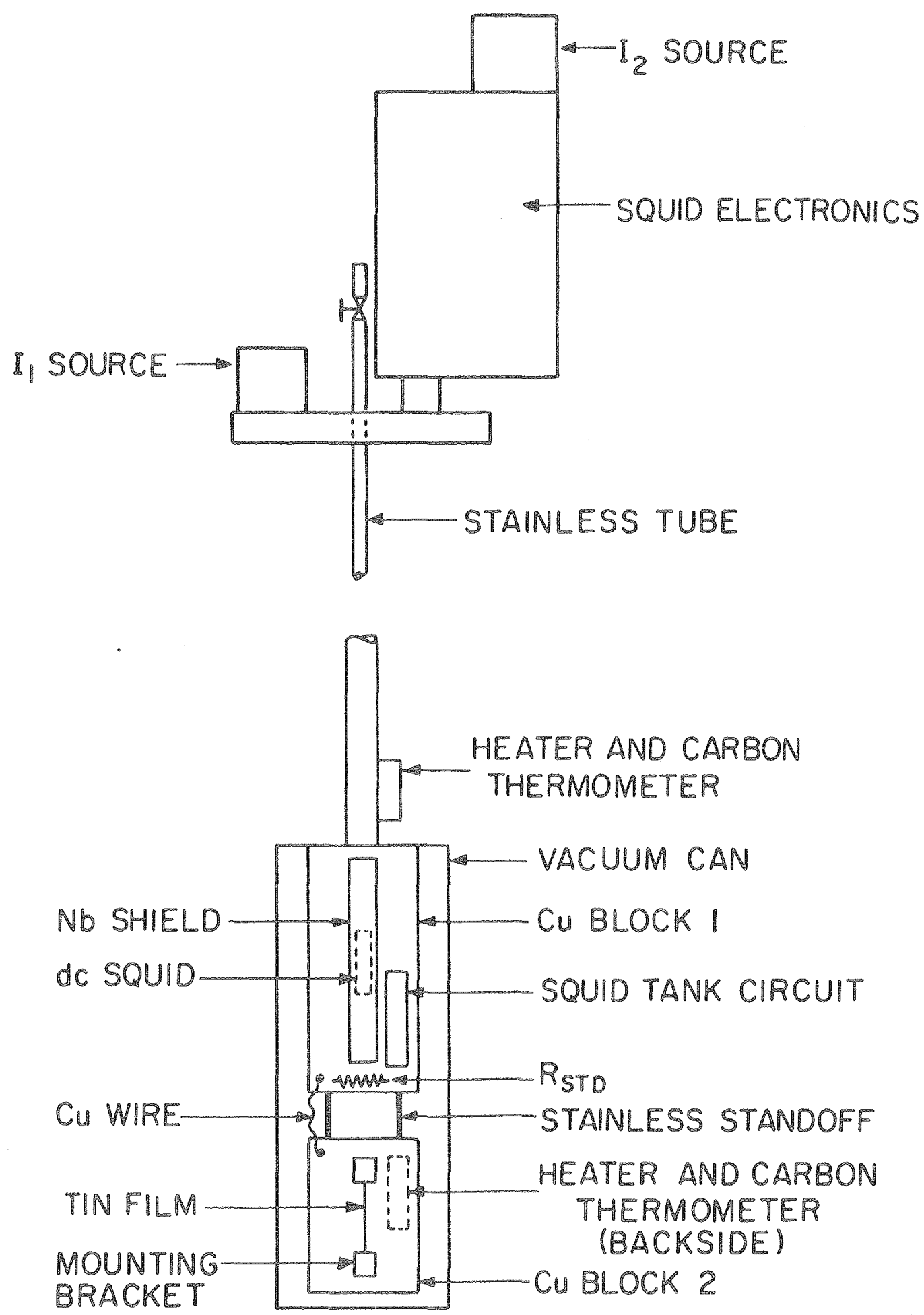

Fig. 19 


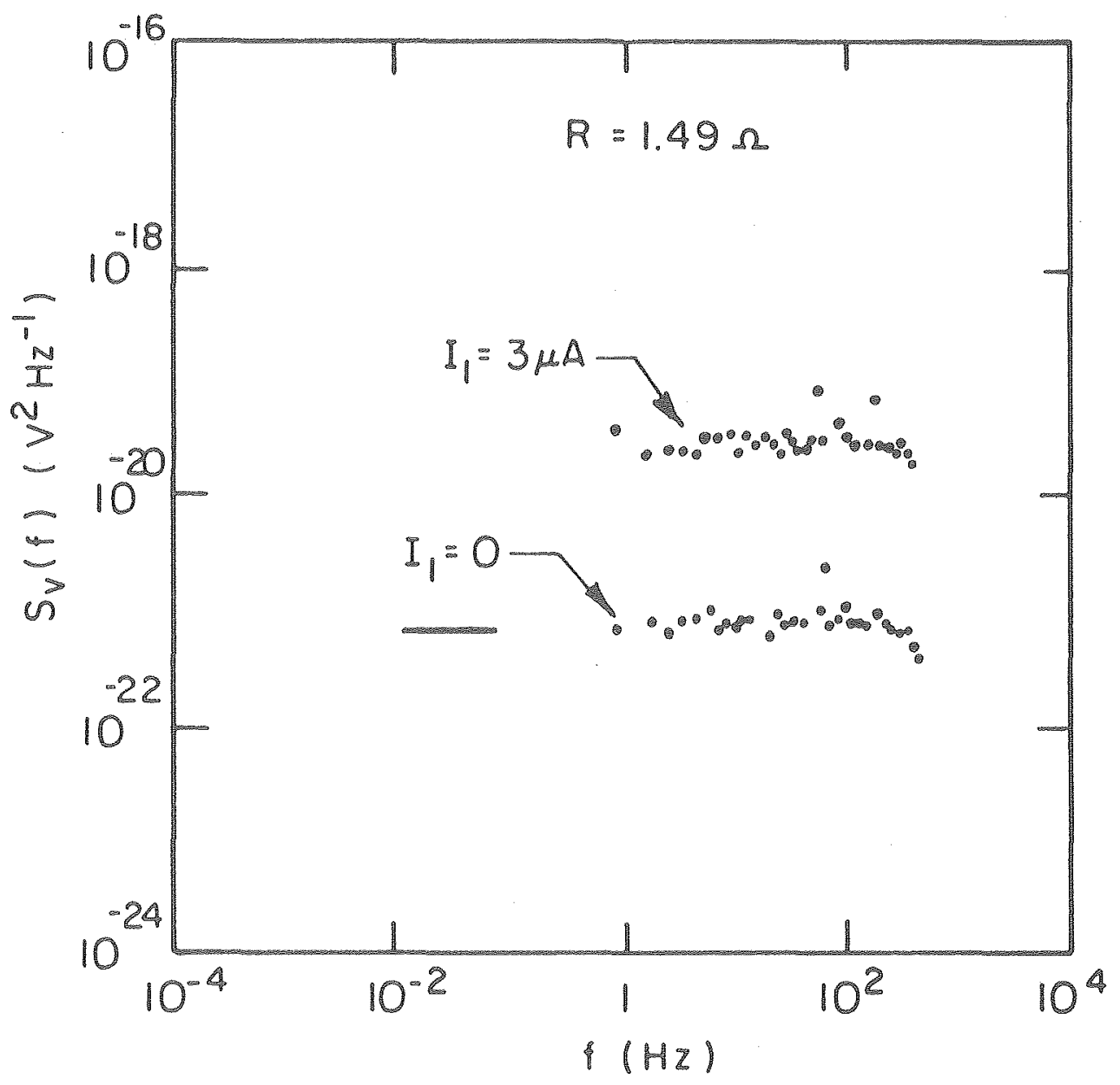

XEL $787-5342$

Fig。 20 


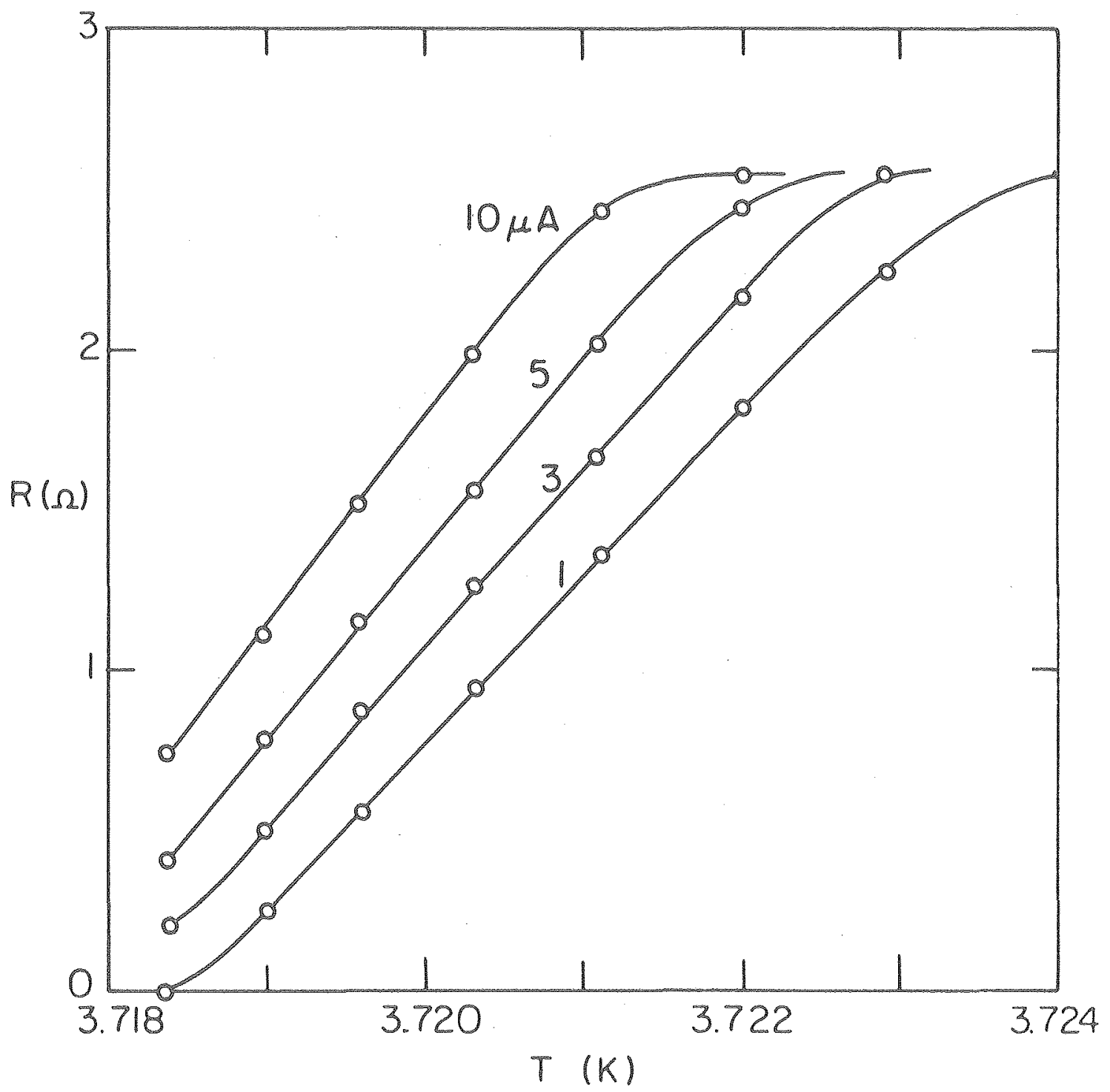

$\times 8 L 787-5341$

Fig. 21 


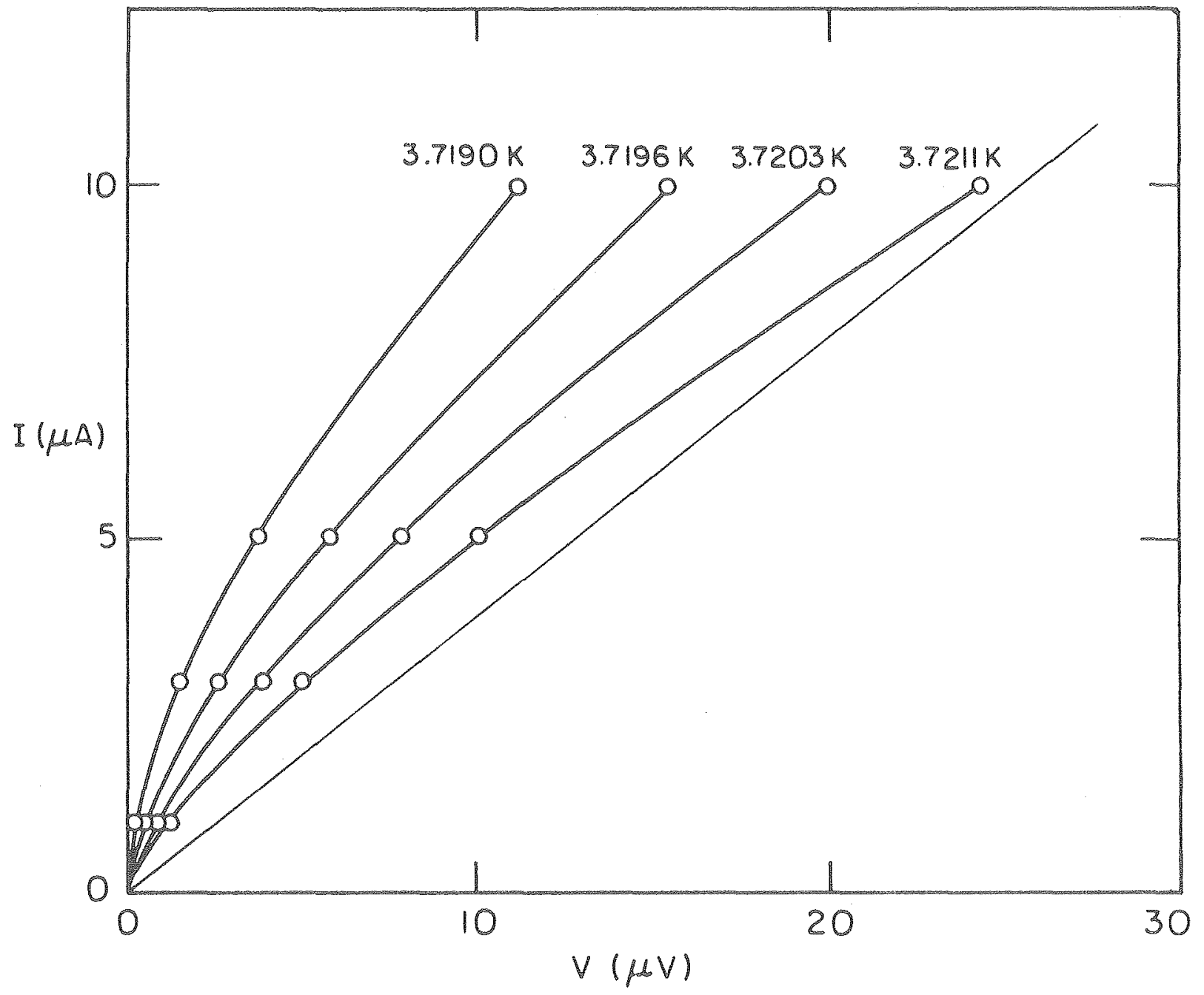

X8L787-5337

Fig. 22 


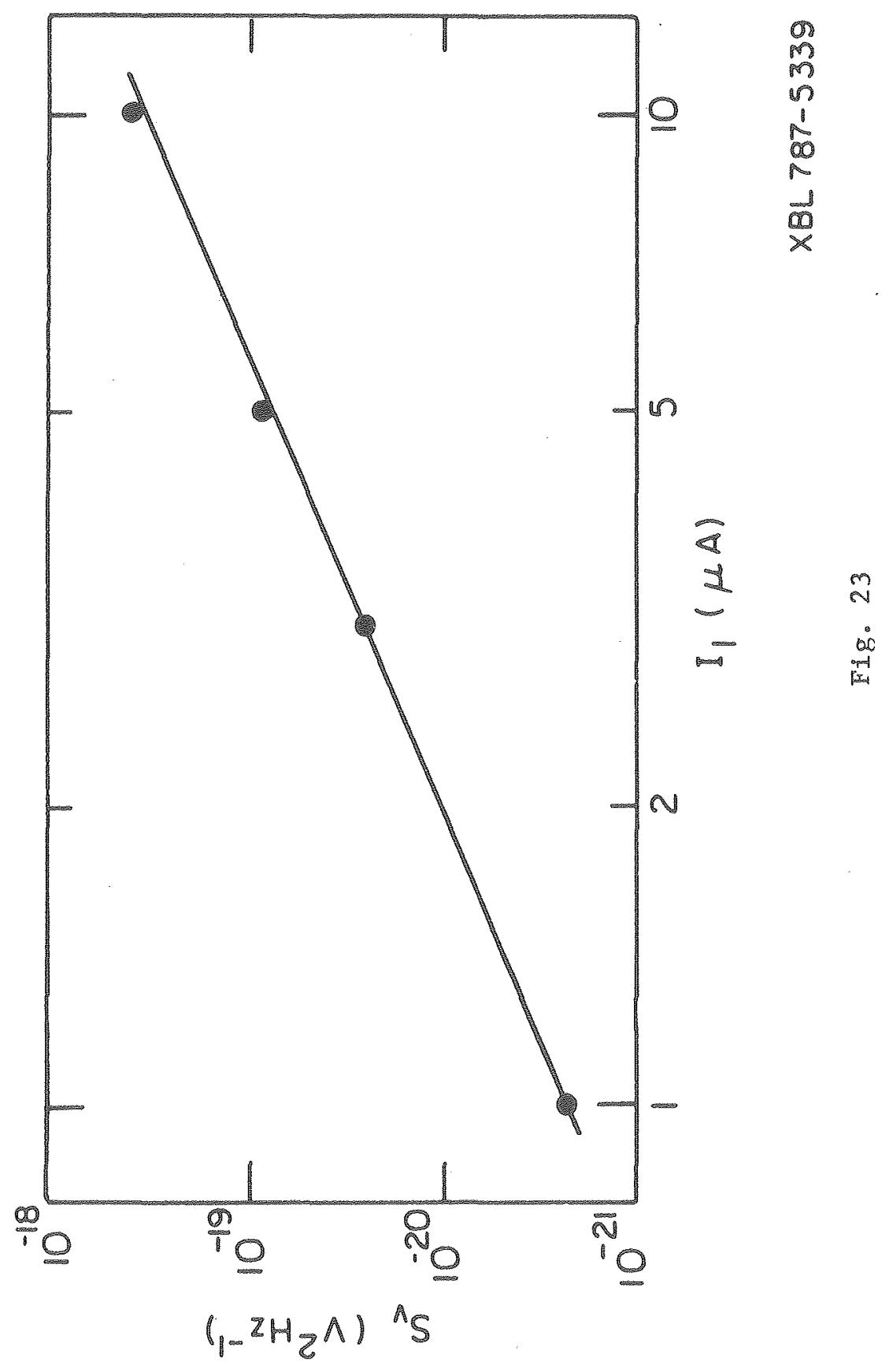


$-102-$

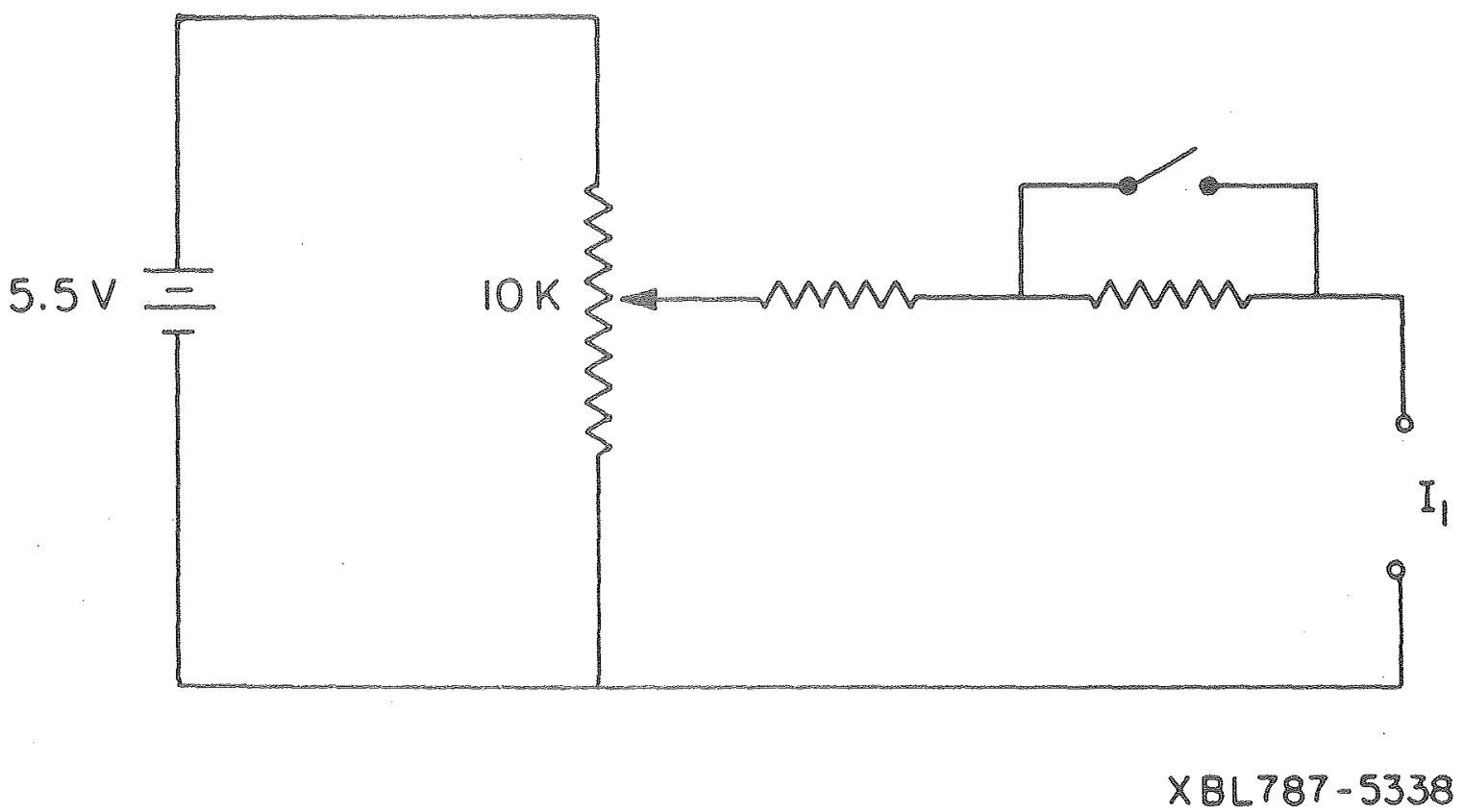

Fig。 24 
$-103-$

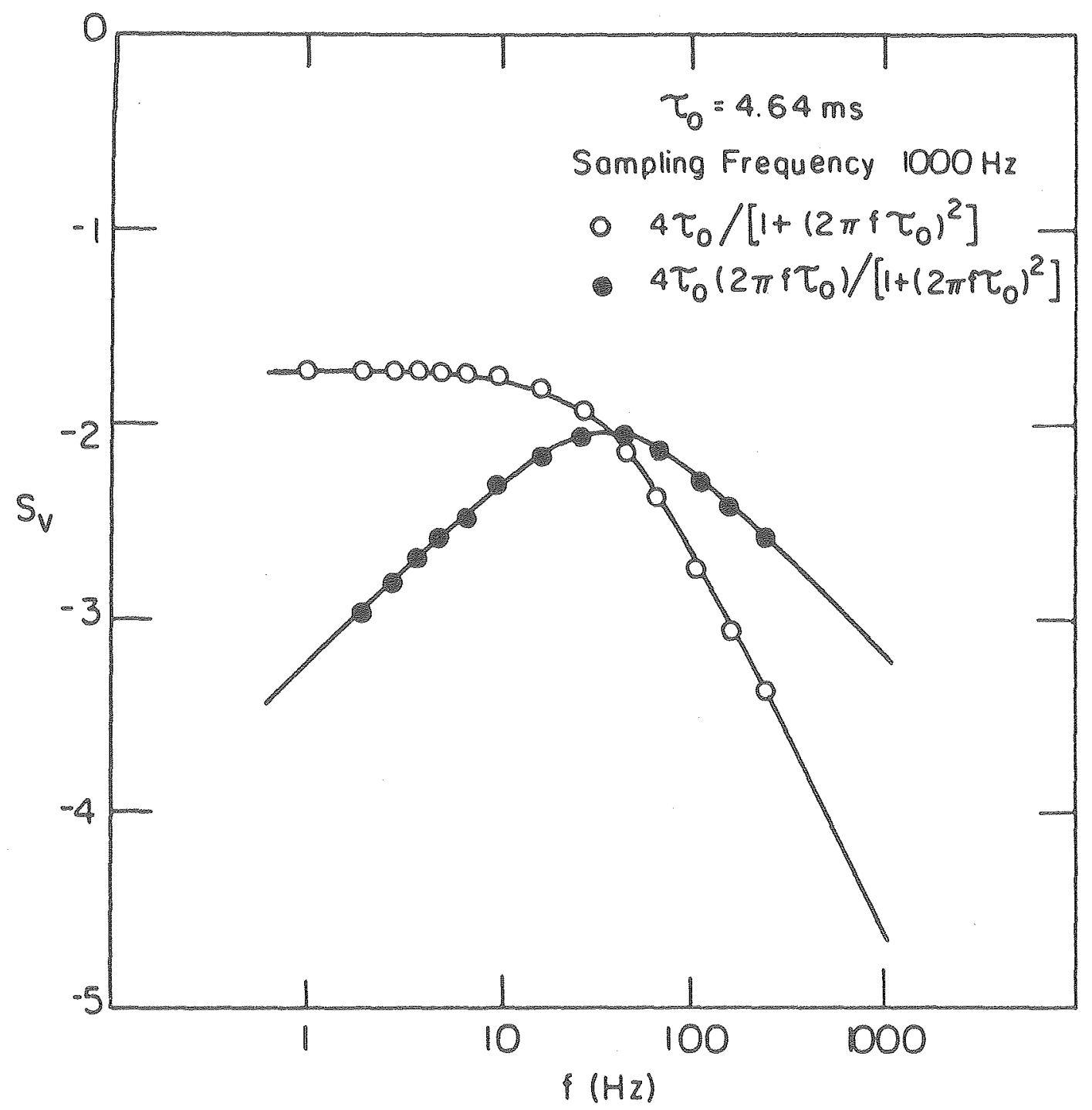

XBL $787-5333$

Fig. 25 


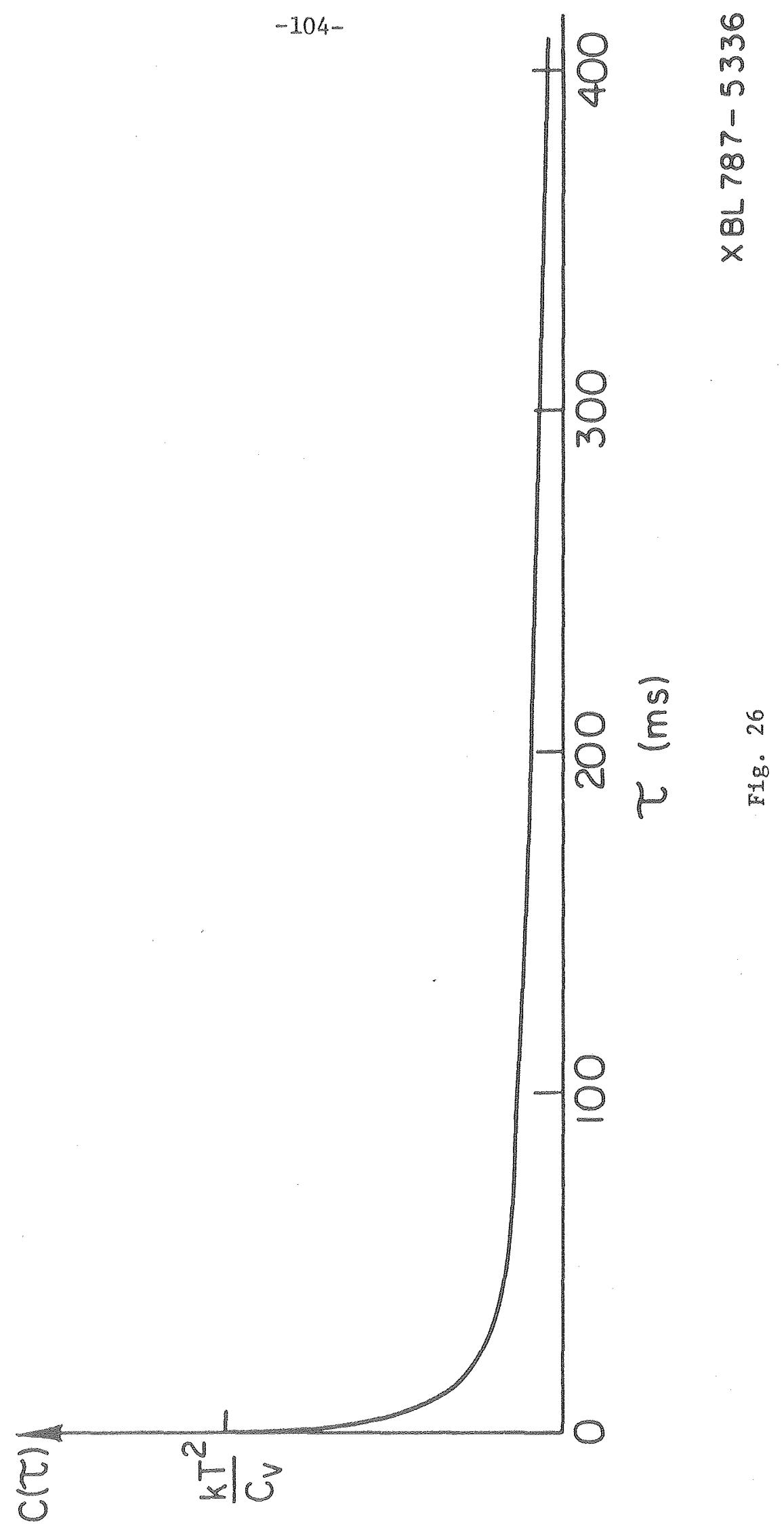




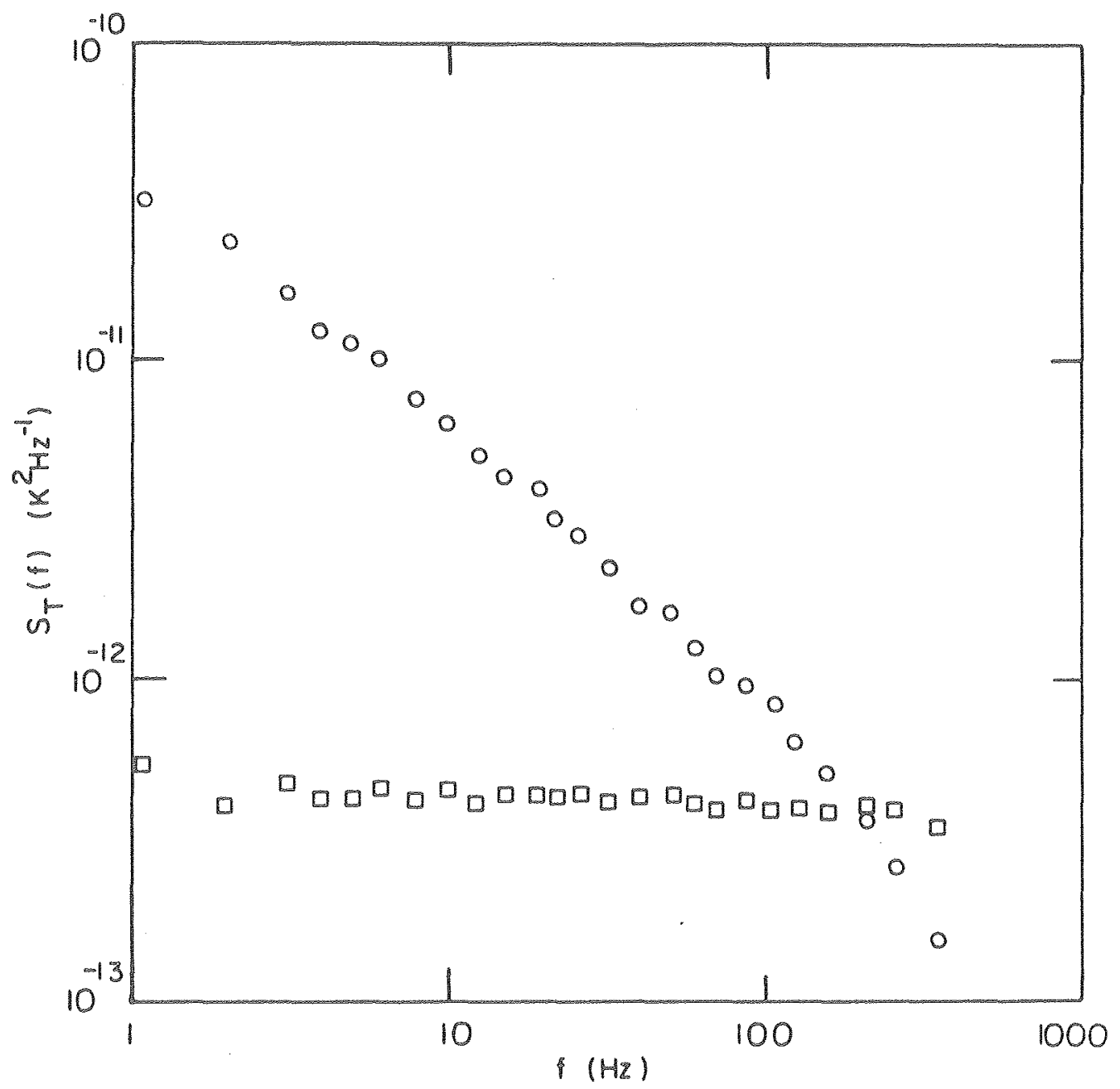

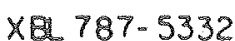

FIg. 27 
This report was done with support from the Department of Energy. Any conclusions or opinions expressed in this report represent solely those of the author(s) and not necessarily those of The Regents of the University of California, the Lawrence Berkeley Laboratory or the Department of Energy. 


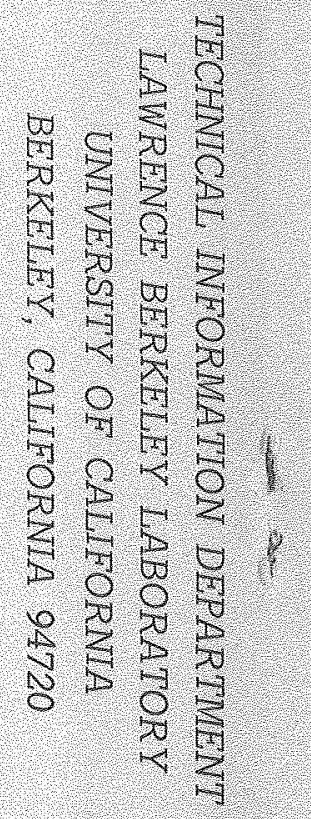

